DRAFT VERSION JANUARY 18, 2017

Preprint typeset using LATEX style AASTeX6 v. 1.0

\title{
KOREAN VLBI NETWORK CALIBRATOR SURVEY (KVNCS): 1. SOURCE CATALOG OF KVN SINGLE DISH FLUX DENSITY MEASUREMENT IN THE K AND Q BANDS
}

Jeong Ae Lee ${ }^{1,2}$, Bong Won Sohn ${ }^{1,2,4}$, Taehyun Jung ${ }^{1,2}$, Do-Young Byun ${ }^{1,2}$ And Jee Won Lee ${ }^{1,3}$

\footnotetext{
${ }^{1}$ Korea Astronomy and Space science Institute, 776, Daedeokdae-ro, Yuseong-gu, Daejeon, Republic of Korea, 34055

${ }^{2}$ Korea University of Science and Technology, 217, Gajeong-ro, Yuseong-gu, Daejeon, Republic of Korea, 34113

${ }^{3}$ Kyunghee University, 1732, Deogyeong-daero, Giheung-gu, Yongin-si, Gyeonggi-do, Republic of Korea, 02447

${ }^{4}$ Corresponding author, bwsohn@kasi.re.kr
}

\begin{abstract}
We present the catalog of the KVN Calibrator Survey (KVNCS). This first part of the KVNCS is a single dish radio survey conducted at 22 (K band) and $43 \mathrm{GHz}$ (Q band) simultaneously using the Korean VLBI Network (KVN) from 2009 to 2011. A total 2045 sources selected from the VLBA Calibrator Survey (VCS) with an extrapolated flux density limit of $100 \mathrm{mJy}$ at K band. The KVNCS contains 1533 sources in the $\mathrm{K}$ band with a flux density limit of $70 \mathrm{mJy}$ and 553 sources in the Q band with a flux density limit of $120 \mathrm{mJy}$; it covers the whole sky down to $-32 .{ }^{\circ} 5$ in declination. Five hundred thirteen sources were detected in the K and Q bands, simultaneously; $~ 76 \%$ of them are flat-spectrum sources $(-0.5 \leq \alpha \leq 0.5)$. From the flux-flux relationship, we anticipated that the most of the radiation of many of the sources comes from the compact components. Therefore, the sources listed in the KVNCS are strong candidates for high frequency VLBI calibrators.
\end{abstract}

Keywords: catalogs - quasars: general - radio continuum: galaxies - surveys

\section{INTRODUCTION}

A large proportion of compact radio sources have flat or inverted spectra (e.g., Kellermann \& Pauliny-Toth 1981; Zensus 1997; Gurvits et al. 1999; Chen \& Wright 2009; de Zotti et al. 2010; Massardi et al. 2011; Mantovani et al. 2011). This implies that these sources are optically thick at the observed radio frequencies. Very long baseline interferometry (VLBI) calibrators are also compact sources. The majority of sources in the Very Long Baseline Array (VLBA) Calibrator Survey (VCS) have flat spectra at 2.3 (S band) and 8.4 (X band) GHz (Beasley et al. 2002; Fomalont et al. 2003; Petrov et al. 2005, 2006; Kovalev et al. 2007; Petrov et al. 2008). However, VLBI calibrators at higher frequency $(>20 \mathrm{GHz})$ are severely rare compared to those at lower frequency. For example, 858 VLBI calibrators in the $\mathrm{K}$ band $(\sim 20 \mathrm{GHz})$ are known (Petrov et al. 2007; Lanyi et al. 2010; Petrov et al. 2011, 2012; Petrov 2012), while $\sim 3800$ sources are listed in the VCS in the S and X bands. High-frequency VLBI observations are useful to understand the physical processes in the vicinity of supermassive black holes of active galactic nuclei (AGN), because a study of optically thin region from the synchrotron radiation of compact radio sources is possible. In addition, radio sources become more compact in structure so that astrometric errors in the celestial reference frame (CRF) would be minimized (e.g. Fey \& Charlot 1997; Ma et al. 1998; Fey et al. 2004; Lanyi et al. 2010; Charlot et al. 2010). Together with the successful operation of the Gaia spacecraft during the first two years of sky survey, high-frequency VLBI sources matching with Gaia samples will be important to compare the radio and optical reference frames (e.g. Bourda et al. 2010, 2011; Jacobs et al. 2014).

There is an extensive blind survey of the southern sky that identified 5808 sources at $20 \mathrm{GHz}$ (AT20G) in order to support subtraction of foreground objects for the measurement of the Cosmic Microwave Background (Murphy et al. 2010). In the northern sky, Righini et al. (2012) conducted the K-band Northern Wide Survey and identified 73 sources at declination $>72 .{ }^{\circ} 3\left(\sim 880\right.$ degree $\left.^{2}\right)$. Recently, Jacobs et al. (2014) constructed a new compact radio survey at X/Ka $(8.4 / 32 \mathrm{GHz})$ bands in order to improve the accuracy of celestial reference frame and detected 631 sources. However, the sky coverage of known $\mathrm{K}$ band calibrators is about $49 \%$ of the whole sky by assuming the circles of $5^{\circ}$ radius as shown in Figure 1, while the VCS sources in the S and X bands cover the full sky above $-40^{\circ}$ in declination with 
this radius (Petrov et al. 2008). The critical separation angle, $5^{\circ}$ was determined, referring to Dodson et al. (2014). They showed a feasibility of source frequency phase referencing (SFPR) in KVN, considering of $5^{\circ} .9$ separation angle between a target and a calibrator in Korean VLBI Network (KVN). We therefore performed 21.7 (K band) and 42.4 (Q band) $\mathrm{GHz}$ single dish survey observations in order to provide a wider sky coverage of possible VLBI target and calibrator sources at those frequencies (or even higher frequencies), particularly for the KVN.

The KVN is composed of three 21-m telescopes in Seoul, Ulsan and Jeju, Korea. Its notable characteristic is the multifrequency receiver system, which makes it possible to observe the radio sources at four different frequencies [22 (K), 43 (Q), 86 (W), and 129 (D bands) GHz] simultaneously (e.g., Han et al. 2008; Lee et al. 2011, 2014). In particular, this system is very efficient for high frequency VLBI observations when the frequency phase transfer (FPT) method is applied to compensate for the atmospheric coherence loss; this method uses lower frequency phase solutions (e.g., in the $\mathrm{K}$ band) to compensate for higher frequency ones (e.g., in the Q/W/D bands), because of the non-dispersive nature of the atmosphere with regard to radio systems (e.g. Jung et al. 2011; Rioja et al. 2014, 2015). As a result, noticeable improvements of coherence time and signal-to-noise ratio (SNR) at high frequency VLBI observations have been demonstrated (e.g. Jung et al. 2012, 2014).

For the practical use of the FPT for higher frequencies (Q/W/D-band) VLBI observations with the KVN, increasing available sources in the $\mathrm{K}$ and $\mathrm{Q}$ bands is very important in the northern sky because the detection of target or calibrator sources at the lower frequencies (e.g. in the $\mathrm{K}$ or $\mathrm{Q}$ bands) as a reference for the atmospheric calibration is essential. The increase of the detection at high frequencies by the FPT will be able to extend our understanding of radio sources from centimeter to millimeter wavelengths in VLBI; for example, a statistical study of a spectral energy distribution (SED) of AGNs based on the simultaneously measured flux densities at a range from 20 to $130 \mathrm{GHz}$, and high frequency astrometric applications (e.g. Rioja et al. 2015). We therefore performed the KVN Calibrator Survey (KVNCS), which aims to measure the single dish flux densities of $\sim 2500$ selected sources in the K and Q bands simultaneously in order to utilize as VLBI calibrators of KVN.

In the next section, we explain the selection criteria for the $\sim 2500$ sources. The observations and data reductions are described in section 3. In section 4, the observational results and their analysis are presented. Finally, we summarize our findings and future prospects in section 5. The full catalog of the KVNCS is also provided in the appendix.

\section{SOURCE SELECTION}

A total of 2503 sources with an extrapolated total flux density (hereafter, $\mathrm{S}_{K}^{S-X}$ ) greater than $100 \mathrm{mJy}$ in the $\mathrm{K}$ band were selected from VCS1 to VCS5 (Beasley et al. 2002; Fomalont et al. 2003; Petrov et al. 2005, 2006; Kovalev et al. 2007). As the most widely used catalog of VLBI calibrators, the VCS contains $\sim 3800$ radio sources that show mostly a compact structure and flat spectra sources with greater than $-40^{\circ}$ in declination. $\mathrm{S}_{K}^{S-X}$ was calculated, assuming a power-law spectrum from the total flux densities in the S and X bands (Sohn et al. 2009). The flux density limit of 100 mJy was a selection criterion, which gives $>8 \sigma$ at a baseline sensitivity of the KVN at K band ${ }^{1}$. A declination limit of $-32 .^{\circ} 5$ was determined so that all sources are observed at more than $20^{\circ}$ above the horizon at transit. For the first observation as a pilot observation, 595 relatively bright sources that have $\mathrm{S}_{K}^{S-X}$ higher than $500 \mathrm{mJy}$ in the $\mathrm{K}$ band were selected, and 493 sources $(\sim 83 \%)$ were successfully detected with the first single dish observations from KVNCS1.0.1 to KVNCS1.1.3 in the K and Q bands. Then, we performed a single dish observation toward 1450 of the remaining 1908 sources, the flux density of which ranges between $100 \mathrm{mJy}$ and $1 \mathrm{Jy}$. In order to avoid concentrated on the specific regions, the 1450 sources were selected by considering the sky coverage of the detected source density, which was calculated by the Delaunay triangulation method $^{2}$, whenever each observation was complete. We assumed that the calibrators are located on the vertex of the triangle in the triangulation method. The target is located in the center of the circumcircle of this triangle, and the radius of this circle become the maximum separation angle between the target and the calibrators.

\section{OBSERVATION AND DATA REDUCTION}

\subsection{Observation}

We selected 2503 sources based on lower frequency VLBI flux density from VCS. A flux densities of 2045 of 2503 sources were measured by single dish observations in the K and Q bands simultaneously using the KVN Yonsei and Ulsan radio telescopes from December 2009 to March 2011. The observational information is summarized in Table

\footnotetext{
${ }^{1}$ KVN status report: http://kvn.kasi.re.kr/status_report/

2 This method was developed by Boris Delaunay in 1934 and is well known in mathematics and computational geometry. The triangles in Delaunay triangulation method minimizes the summation of the interior angles of the triangles. In addition, these triangles do not include any points except the vertices within their circumcircle (from Wikipedia).
} 
1. The cross-scan mode (cs-mode), a one-dimensional on-the-fly method, was used to obtain an accurate flux density measurement. A single cs-mode scan consists of two scans in azimuth (Az) and two in elevation (El). Each scan includes both forward and backward scans. We selected the parameters for the cs-mode considering the telescope beam size, time scales of the sky power variation, and a hardware and software limitations of the KVN system. The applied scan speed, the data sampling interval and the scan length are mainly $65^{\prime \prime} / \mathrm{sec}, 0.1 \mathrm{~ms}$, and $13^{\prime}$, respectively, which yield 4 (K band) and 2 (Q band) seconds full-beam cross time. We can remove sky power variation of which time scale is longer than the full-beam cross time by fitting off-source data. Assuming a Gaussian beam pattern, the on-source integration time of a cross scan are 8 and 4 seconds in the $\mathrm{K}$ and $\mathrm{Q}$ bands, respectively. The total on-source time for each source is considered based on the estimated source flux density and is calculated by multiplying by the number of scans. The main-beam sizes of the KVN telescopes are around $126^{\prime \prime}$ and $63^{\prime \prime}$ in the K and Q bands, respectively ${ }^{3}$. The mean beam sizes in the $\mathrm{K}$ and $\mathrm{Q}$ bands are quite consistent with the known ones. However, the estimated beam sizes and pointing offsets have large errors for faint sources and under the bad weather conditions or rapid sky variation (e.g., Fante 1975). We used them to eliminate low-quality data with $30 \%$ differences in beam size from the known ones and with the pointing offsets larger than $25^{\prime \prime}$ arbitrarily. The standard deviations in the beam size and pointing offset data are 7\% (K) and 20\% (Q) and $4 "(\mathrm{~K})$ and $4 "(\mathrm{Q})$ in Az and $10 \%(\mathrm{~K})$ and $19 \%(\mathrm{Q})$ and 6.4 " (K) and 6.0" (Q) in El, respectively. The observing frequencies were $21.7 \mathrm{GHz}$ for the $\mathrm{K}$ band and $42.4 \mathrm{GHz}$ for the Q band with $512 \mathrm{MHz}$ bandwidth. A measured root-mean-square (RMS) error is an order of magnitude higher than estimated thermal one. This is usual in practice. Hot/cold load calibration was performed in order to determine the antenna temperature scale from KVNCS1.0.1 to KVNCS1.2.1. For hot/cold load calibration, we used two microwave absorbers, one in room temperature of $\sim 292 \mathrm{~K}$ and the other immersed in liquid nitrogen of $80 \mathrm{~K}$, as hot and cold loads, respectively. From KVNCS1.2.2, we used chopper-wheel method which uses a sky as a cold load together with a microwave absorber in room temperature (Kutner \& Ulich 1981; Mangum 2000). The results from these two calibration methods were consistent each other within $\sim 2 \%$ uncertainty. The system temperature and zenith optical depth of each observation are presented in Table 1. The sky opacity was corrected using the sky-dipping method based on observations every hour. 3C 286 was observed as a flux calibrator every $1.5 \mathrm{~h}$ to convert the measured antenna temperature into the flux. The flux densities of 3C 286 are 2.64 and $1.51 \mathrm{Jy}$ in the K and Q bands, respectively. These were measured with a brightness model of Mars at the KVN Yonsei observatory (Sohn, in prep.).

\subsection{Data reduction}

We developed an analysis program for the KVN single dish flux density measurements and the following procedures were applied: (a) Extraction of bad scans due to the weather conditions or instrumental spuriousness, (b) linear baseline fitting to estimate the RMS noise level and to eliminate sky level, (c) Gaussian fitting to measure the antenna temperature and to correct pointing offsets deviating from the Gaussian fitted center position using equations (1) and (2) (Fuhrmann 2004).

$$
\begin{aligned}
& \left(T_{a, A z}^{*}\right)^{\prime}=T_{a, A z}^{*} \cdot \exp \left[4 \ln 2 \frac{x_{E l}^{2}}{\theta_{E l}^{2}}\right] \\
& \left(T_{a, E l}^{*}\right)^{\prime}=T_{a, E l}^{*} \cdot \exp \left[4 \ln 2 \frac{x_{A z}^{2}}{\theta_{A z}^{2}}\right]
\end{aligned}
$$

$T_{a, A z}^{*}$ and $T_{a, E l}^{*}$ are the measured antenna temperature corrected for atmospheric attenuation along the $\mathrm{Az}$ and $\mathrm{El}$ axes in the cs-mode, respectively. Further, $x_{A z}$ and $x_{E l}$ are pointing offsets, and $\theta_{A z}$ and $\theta_{E l}$ are the half-power beam width in arcseconds. After the pointing correction, $\left(T_{a, A z}^{*}\right)^{\prime}$ and $\left(T_{a, E l}^{*}\right)^{\prime}$ were averaged. (d) The error propagation of the antenna temperature was calculated using equation (3).

$$
\sigma_{\left(T_{a}^{*}\right)^{\prime}}=\left(T_{a}^{*}\right)^{\prime} \sqrt{\left[\frac{\sigma_{\Delta G}^{2}}{\Delta G^{2}}+\frac{\sigma_{T_{a}^{*}}^{2}}{\left(T_{a}^{*}\right)^{2}}\right]}
$$

$\left(T_{a}^{*}\right)^{\prime}$ is the corrected and averaged $T_{a}^{*}$ for the pointing offset and in Az and El. $\sigma_{T_{a}^{*}}$ represents the uncertainty of $T_{a}^{*}$. $\Delta \mathrm{G}$ is the temporal variation of the antenna gain, and its uncertainty is written as $\sigma_{\Delta G}$. The gain curves of the KVN radio telescopes are very flat for elevations of 20 to $80^{\circ}$ in the $\mathrm{K}$ and $\mathrm{Q}$ bands. The gain variation along this elevations were $2.5 \%(\mathrm{~K})$ and $2.0 \%(\mathrm{Q})$ at KYS and $1.5 \%(\mathrm{~K})$ and $4.5 \%(\mathrm{Q})$ at KUS ${ }^{3}$. Thus, the elevation dependence of the gain variation was ignored. $\Delta \mathrm{G}$ was obtained by using the ratio of the mean $\left(T_{a}^{*}\right)^{\prime}$ to $\left(T_{a}^{*}\right)^{\prime}$ of $3 \mathrm{C} 286$. However, the

\footnotetext{
${ }^{3}$ KVN status report: http://kvn.kasi.re.kr/status_report/
} 
error was dominated by the thermal random noise. The flux density conversion factors were determined from the ratio of the flux densities to $\left(T_{a}^{*}\right)^{\prime}$ of $3 \mathrm{C} 286$ in the K and Q bands (see Table 1), which were obtained using the NRAO Mars emission model (Butler et al. 2001). To check the results, we compared these conversion factors with those obtained for planets (Lee et al. 2011), which have $10 \%$ uncertainty, and found that they are consistent within $10 \%$ and $8 \%$ in the $\mathrm{K}$ and $\mathrm{Q}$ bands, respectively. Finally, the conversion factors obtained for $3 \mathrm{C} 286$ were applied to estimate the source flux densities.

\section{RESULTS AND DISCUSSION}

\subsection{Flux density measurement}

Among 2043 sources, the flux densities of 1533 (75\%) and 533 (27\%) sources with $3 \sigma$ noise levels of 66 (K band) and 108 (Q band) mJy were successfully measured. Their median flux densities were 397 and 588 mJy and the lowest flux densities were $\sim 70$ and $\sim 120 \mathrm{mJy}$ in the $\mathrm{K}$ and $\mathrm{Q}$ bands, respectively. Among them, the flux densities of 513 sources were measured in the $\mathrm{K}$ and $\mathrm{Q}$ bands simultaneously. The distribution of the measured flux densities are shown in Figure 2 and the measured flux densities are listed in Table 2.

The luminosity distribution of 1138 sources as a function of the redshift $(z)$ are plotted in Figure 3 . Archival redshifts were taken from NASA/IPAC Extragalactic Database (NED) and the Sloan Digital Sky Survey (SDSS) DR13. Only 23 sources were given as the photometric redshifts and their mean uncertainty are about 0.618. These luminosities are calculated with $H_{0}=73 \mathrm{~km} \mathrm{~s}^{-1} M_{p c}^{-1}$ and $\Omega_{m}=0.27$ at $21.7 \mathrm{GHz}{ }^{4}$, according to the arbitrarily spectral indices $(\alpha$ $=-1.0$ and $0.0, S \sim \nu^{\alpha}$, where $S$ is the flux, and $\nu$ is the observed frequency). We denote spectra as steep $(\alpha<-0.5)$, flat $(-0.5 \leq \alpha \leq 0.5)$ and inverted $(\alpha>0.5)$ in this study. This figure reflects that our data are those of flux-limited samples, and they show the distribution of high-power radio sources, which ranges from $10^{24}$ to $10^{29} \mathrm{~W} \mathrm{~Hz}^{-1}$ at 21.7 $\mathrm{GHz}$ (the observing frequency). According to the number distribution with respect to $z$ in the bottom right panel in Figure 3, the peak is located at the $z \sim 1$. That of bright quasars is known to be around $z \sim 1$ (White et al. 2000). In addition, these distributions in four- $z$ bins are shown in Figure 4. Gray-filled and black-hatched bars indicate each distribution according to $\alpha=-1.0$ and 0.0 , respectively. The distributions according to $\alpha$ are similar in the low- $z$ region $(z<0.5)$, whereas they differ in the high- $z$ region $(z \geq 0.5)$.

\subsection{Comparison with the VLBI flux densities in the $S, X, K$, and $Q$ bands}

We compared the VCS flux densities in the S and X bands with those of the KVNCS in the K and Q bands, because large difference, if ever, between the observed flux densities in the $\mathrm{K}$ and $\mathrm{Q}$ bands and the extrapolated flux densities in the S and X bands would mean VLBI missing flux problem, high source variability or source evolution (e.g., opacity changes). Their correlations with the weighted linear fit lines are shown in Figure 5. The linear correlation coefficients are $0.77(\mathrm{~S}-\mathrm{K}), 0.87(\mathrm{X}-\mathrm{K}), 0.81(\mathrm{~S}-\mathrm{Q})$, and $0.85(\mathrm{X}-\mathrm{Q})$. The linear fit lines are obtained using the data having a K-band flux density greater than 397 mJy (median flux) and less than 1Jy (arbitrarily) and a Q-band flux density greater than $587 \mathrm{mJy}$ (median flux), because the data contain faint sources that were not detected at high frequency (e.g., the $\mathrm{K}$ or $\mathrm{Q}$ bands) but were detected at low frequency (e.g., the $\mathrm{S}$ or $\mathrm{X}$ bands). This causes a selection effect for non-detected sources (the red and black arrows), which are faint sources with a flux density less than $3 \sigma$. Thus, the sources less than the median flux density were excepted for fitting. In addition, the bright sources ( $>$ 1Jy) in the $\mathrm{K}$ band excluded for fitting because they can always be detected, although they are highly variable. The slopes of the weighted linear fit lines are $1.04 \pm 0.024$ and $0.79 \pm 0.005$ for the $\mathrm{S}-\mathrm{K}$ and $\mathrm{S}-\mathrm{Q}$ bands, respectively, whereas they are $0.63 \pm 0.024(\mathrm{X}-\mathrm{K})$ and $0.59 \pm 0.005(\mathrm{X}-\mathrm{Q})$. The differences between the slopes in the $\mathrm{S}$ and $\mathrm{X}$ bands imply that there are missing flux densities in the $\mathrm{X}$ band. The flux densities measured from the VCS were calculated from the CLEAN components from VLBI observations and the flux densities for some sources were obtained within 7 (S band) and 25 (X band) $\mathrm{M} \lambda$ in projected uv-distance (Petrov et al. 2006; Kovalev et al. 2007). Therefore, there is missing flux density in the $\mathrm{X}$ bands, depending on the structure of each source.

We also compared the flux densities of 232 (K band) and 76 (Q band) common sources from the KVNCS and VLBI imaging survey for the International Celestial Reference Frame at 24 and $43 \mathrm{GHz}$ (Charlot et al. 2010). Figure 6 shows their flux-flux relationships in the K and Q bands. On the basis of the structure index (SI) from Charlot et al. (2010), $195(84 \%)$ and $63(83 \%)$ sources were identified as SI < 3 (compact sources), and the remaining $37(16 \%)$ and $13(17 \%)$ sources showed SI $\geq 3$ (sources with marginally compact or extended structure) in the K and Q bands, respectively. To obtain the weighted linear fit results, the data were selected in the same way as those in Figure 5 . The linear fit

${ }^{4}$ http://ned.ipac.caltech.edu/ 
at SI $<3$ was performed for source flux densities greater than 397 mJy and less than 1 Jy. However, some compact sources show the big differences of flux density between VLBI and single dish. For instance, a source which has $\sim 4$ Jy of VLBI and $\sim 1$ Jy of single dish flux densities has been known as a variable, J0238+1636 which had big flux variations of around 5 Jy from 2007 to $2010^{5}$. In addition, a source which has $\sim 0.3$ Jy (VLBI) and $\sim 3$ Jy (single dish) is $\mathrm{J} 1849+6705$. Also this has been known as a variable which shows year-scale variations ${ }^{5}$. Therefore, these variables were not considered to fit in the $\mathrm{K}$ band. At SI $\geq 3$, on the other hand, the linear fit results were calculated with all sources because the number of sources was small to fit. The slope for the compact sources is $1.07 \pm 0.065$, while that of extended sources is $0.66 \pm 0.006$ in the $\mathrm{K}$ band. We infer that most of the radiation from the compact sources (SI $<3$ ) comes from the compact core region, whereas the extended sources ( $\mathrm{SI} \geq 3$ ) have missing flux. In the $\mathrm{Q}$ band, because there are few common sources, the weighted linear fit was applied to all 76 sources. We expect that most of the radiation ( $\sim 80 \%)$ comes from the compact component, although about $20 \%$ of the flux density in the $\mathrm{Q}$ band is missing.

\subsection{Spectral index distributions}

Table 3 shows the statistics of $\alpha$ for 1533 sources in the $\mathrm{K}$ band and 553 sources in the Q band, and 513 sources detected in the $\mathrm{K}$ and $\mathrm{Q}$ bands simultaneously. As expected, many of our sources have flat spectra in both the $\mathrm{S}$ and $\mathrm{X}$ and the $\mathrm{K}$ and $\mathrm{Q}$ bands. The distributions of the spectral indices of the $\mathrm{S}$ and $\mathrm{X}\left(\alpha_{S X}\right), \mathrm{X}$ and $\mathrm{K}\left(\alpha_{X K}\right), \mathrm{X}$ and $\mathrm{Q}$ $\left(\alpha_{X Q}\right)$, and $\mathrm{K}$ and $\mathrm{Q}\left(\alpha_{K Q}\right)$ bands are shown in Figure 7. The top panel shows the distributions of $\alpha_{S X}$ and $\alpha_{X K}$ for 1533 sources and $\alpha_{X Q}$ for 553 sources. The distribution of $\alpha_{X K}$ become broader than that of $\alpha_{S X}$, but $\sim 88 \%$ of the $\alpha_{S X}$ values and $\sim 70 \%$ of the $\alpha_{X K}$ values belong to the flat spectrum ranges. In addition, the distribution of $\alpha_{X Q}$ is similar, and we expect that these are relatively bright sources. In the bottom panel, the distribution of $\alpha_{X K}$ is broader than the other distributions and shifts toward the inverted spectrum region because these sources are corrupted by flux density variability. There is an observational epoch gap between the VCS and KVNCS. However, $\alpha_{X Q}$ shows a distribution similar to that of $\alpha_{S X}$. These sources are sufficiently bright. In addition, that of $\alpha_{K Q}$ is steeper $(\sim 14 \%)$ than those of $\alpha_{S X}(\sim 6 \%), \alpha_{X K}(\sim 6 \%)$, and $\alpha_{X Q}(\sim 3 \%)$. We infer that the sources become relatively optically thin in the $\mathrm{K}$ and $\mathrm{Q}$ bands. Nevertheless, $76 \%$ of the sources show flat spectra in the K and Q bands.

\subsection{Sky coverage}

Figure 8 clearly shows that our sample is fairly evenly distributed on the sky (this figure is the same as Figure 1 in Lee et al. (2012)). Assuming a spatial coherence scale of $5^{\circ}$, which is the separation angle between a target and the calibrators, $99 \%$ of the sky is covered by these sources above $-32 .^{\circ} 5$ in declination (Figure 9 ). The sky coverage is improved by more than $20 \%$ compared to that of the existing calibrators shown in Figure 1. In the same way, if the spatial coherence scales are assumed as 2.2 and $3^{\circ}$, the sky coverages are improved about 28 and $38 \%$, respectively. $2.2^{\circ}$ means a maximum separation of VERA dual-beam ${ }^{6}$ which is able to observe a target and a calibrator simultaneously and $3^{\circ}$ is a typical coherence scale in the $\mathrm{K}$ band. Hence, it is expected that there will have the possibility of improving the sky coverage of the $\mathrm{K}$ band calibrators with a uniform distribution, comparing to the existing one (Figure 1).

\section{SUMMARY}

We conducted an extensive single dish survey (the KVNCS) of 2043 extragalactic radio sources in the K and Q bands using the KVN and successfully detected 1533 (75\%) of the sources in the K band and $553(27 \%)$ in the Q band. In addition, the flux densities of 513 sources were measured in the K and Q bands, simultaneously. This catalog is an important database for high frequency VLBI observations with the KVN and other available radio telescopes worldwide. In addition, these sources become VLBI calibrator candidates. The sky density distribution of the 1533 sources covered about $99 \%$ of the sky observable by the KVN. About $76 \%$ of the 513 sources still showed flat spectra in the $\mathrm{K}$ and $\mathrm{Q}$ bands. On the basis of the flux-flux relationship between the single dish survey and VLBI observations, we inferred that most of the radiation of many of the sources comes from the compact components. To confirm the feasibility of using these sources as reliable VLBI phase calibrators, however, VLBI fringe and imaging surveys should be performed. These VLBI follow-ups are ongoing with the KVN and KaVA (KVN and VERA Array) in the K band. In addition, a simultaneous multiwavelength Active Galactic Nuclei survey is in progress.

The authors appreciate the support of DRC program of Korea Research Council of Fundamental Science and Tech-

${ }^{5}$ F-GAMMA project: http://www3.mpifr-bonn.mpg.de/div/vlbi/fgamma/fgamma.html

${ }^{6}$ http://veraserver.mtk.nao.ac.jp/system/dualbeam-e.html 
nology (FY 2013). We are grateful to all staff members at the KVN who helped to operate the array and to correlate the data. The KVN is a facility operated by KASI (Korea Astronomy and Space Science Institute). KVN operations are supported by KREONET (Korea Research Environment Open NETwork) which is managed and operated by KISTI (Korea Institute of Science and Technology Information). The authors are grateful for the valuable comments of the anonymous referee.

\section{REFERENCES}

Beasley, A. J., Gordon, D., Peck, A. B., et al. 2002, ApJS, 141, 13

Bourda, G., Charlot, P., Porcas, R. W., \& Garrington, S. T. 2010, A\&A, 520, A113

Bourda, G., Collioud, A., Charlot, P., Porcas, R., \& Garrington, S. 2011, A\&A, 526, A102

Butler, B. J., Steffes, P. G., Suleiman, S. H., Kolodner, M. A., \& Jenkins, J. M. 2001, Icarus, 154, 226

Charlot, P., Boboltz, D. A., Fey, A. L., et al. 2010, AJ, 139, 1713

Chen, X., \& Wright, E. L. 2009, ApJ, 694, 222

de Zotti, G., Massardi, M., Negrello, M., \& Wall, J. 2010, A\&A Rv, 18, 1

Dodson, R., Rioja, M. J., Jung, T.-H., et al. 2014, AJ, 148, 97

Fante, R. L. 1975, IEEE Proceedings, 63, 1669

Fey, A. L., \& Charlot, P. 1997, ApJS, 111, 95

Fey, A. L., Ma, C., Arias, E. F., et al. 2004, AJ, 127, 3587

Fomalont, E. B., Petrov, L., MacMillan, D. S., Gordon, D., \& Ma, C. 2003, AJ, 126, 2562

Fuhrmann, L. 2004, dissertation, University of Bonn, Germany

Gurvits, L. I., Kellermann, K. I., \& Frey, S. 1999, A\&A, 342, 378

Han, S.-T., Lee, J.-W., Kang, J., et al. 2008, International Journal of Infrared and Millimeter Waves, 29, 69

Jacobs, C. S., Clark, J. E., Garcí-Miró, C., et al. 2014, in 8th IVS General Meeting, Shanghai, China, ed. D. Behrend, K. D. Baver, \& K. L. Armstrong

Jung, T., Sohn, B. W., \& Byun, D. Y. 2012, in Proceedings of the 11th European VLBI Network Symposium Users Meeting. 9-12 October, 2012. Bordeaux (France), 60

Jung, T., Sohn, B. W., \& Byun, D. Y. 2014, in Proceedings of the 12th European VLBI Network Symposium and Users Meeting 7-10 October 2014. Cagliari, Italy., 86

Jung, T., Sohn, B. W., Kobayashi, H., et al. 2011, PASJ, 63, 375

Kellermann, K. I., \& Pauliny-Toth, I. I. K. 1981, ARA\&A, 19, 373

Kovalev, Y. Y., Petrov, L., Fomalont, E. B., \& Gordon, D. 2007, AJ, 133, 1236

Kutner, M. L., \& Ulich, B. L. 1981, ApJ, 250, 341
Lanyi, G. E., Boboltz, D. A., Charlot, P., et al. 2010, AJ, 139, 1695

Lee, J. A., Sohn, B. W., Jung, T., Byun, D. Y., \& Lee, J. W. 2012, in Proceedings of the 11th European VLBI Network Symposium Users Meeting. 9-12 October, 2012. Bordeaux (France)., 28

Lee, S.-S., Byun, D.-Y., Oh, C. S., et al. 2011, PASP, 123, 1398

Lee, S.-S., Petrov, L., Byun, D.-Y., et al. 2014, AJ, 147, 77

Ma, C., Arias, E. F., Eubanks, T. M., et al. 1998, AJ, 116, 516

Mangum, J. G. 2000, User's Manual for the NRAO 12 Meter Millimeter-Wave Telescope, National Radio Astronomy Observatory, Tucson,Arizona

Mantovani, F., Bondi, M., \& Mack, K.-H. 2011, A\&A, 533, A79

Massardi, M., Ekers, R. D., Murphy, T., et al. 2011, MNRAS, 412,318

Murphy, T., Sadler, E. M., Ekers, R. D., et al. 2010, MNRAS, 402, 2403

Petrov, L. 2012, MNRAS, 419, 1097

Petrov, L., Hirota, T., Honma, M., et al. 2007, AJ, 133, 2487

Petrov, L., Honma, M., \& Shibata, S. M. 2012, AJ, 143, 35

Petrov, L., Kovalev, Y. Y., Fomalont, E. B., \& Gordon, D. 2005, AJ, 129, 1163

-. 2006, AJ, 131, 1872

-. 2008, AJ, 136, 580

-. 2011, AJ, 142, 35

Righini, S., Carretti, E., Ricci, R., et al. 2012, MNRAS, 426, 2107

Rioja, M. J., Dodson, R., Jung, T., \& Sohn, B. W. 2015, AJ, 150,202

Rioja, M. J., Dodson, R., Jung, T., et al. 2014, AJ, 148, 84

Sohn, B. W., Oh, K. S., Jung, M. Y., Jung, T. H., \& Lee, S. S. 2009, Astronomische Nachrichten, 330, 301

White, R. L., Becker, R. H., Gregg, M. D., et al. 2000, ApJS, 126,133

Zensus, J. A. 1997, ARA\&A, 35, 607 


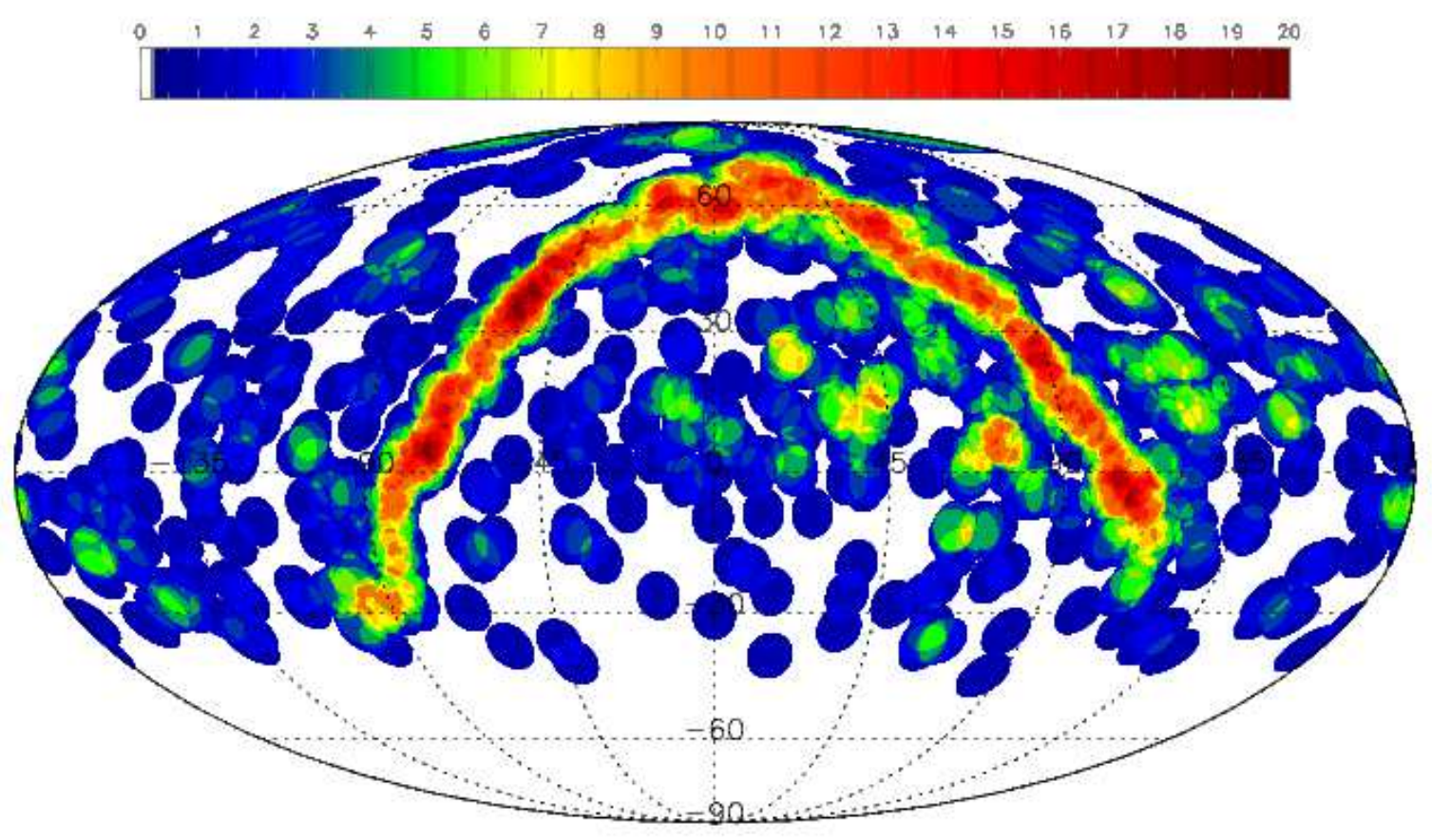

Figure 1. Spatial distribution of 858 sources detected in the K band from Petrov et al. (2007), Lanyi et al. (2010), Petrov et al. (2011), Petrov et al. (2012) and Petrov (2012). The radius of each circle is $5^{\circ}$, which is the separation angle between sources. The colored legend shows the values represented by overlapping circles. This map is drawn using a Mollwide equal-area projection (abscissa: right ascension $\left[{ }^{\circ}\right]$, ordinate: declination $\left[{ }^{\circ}\right]$ ). 

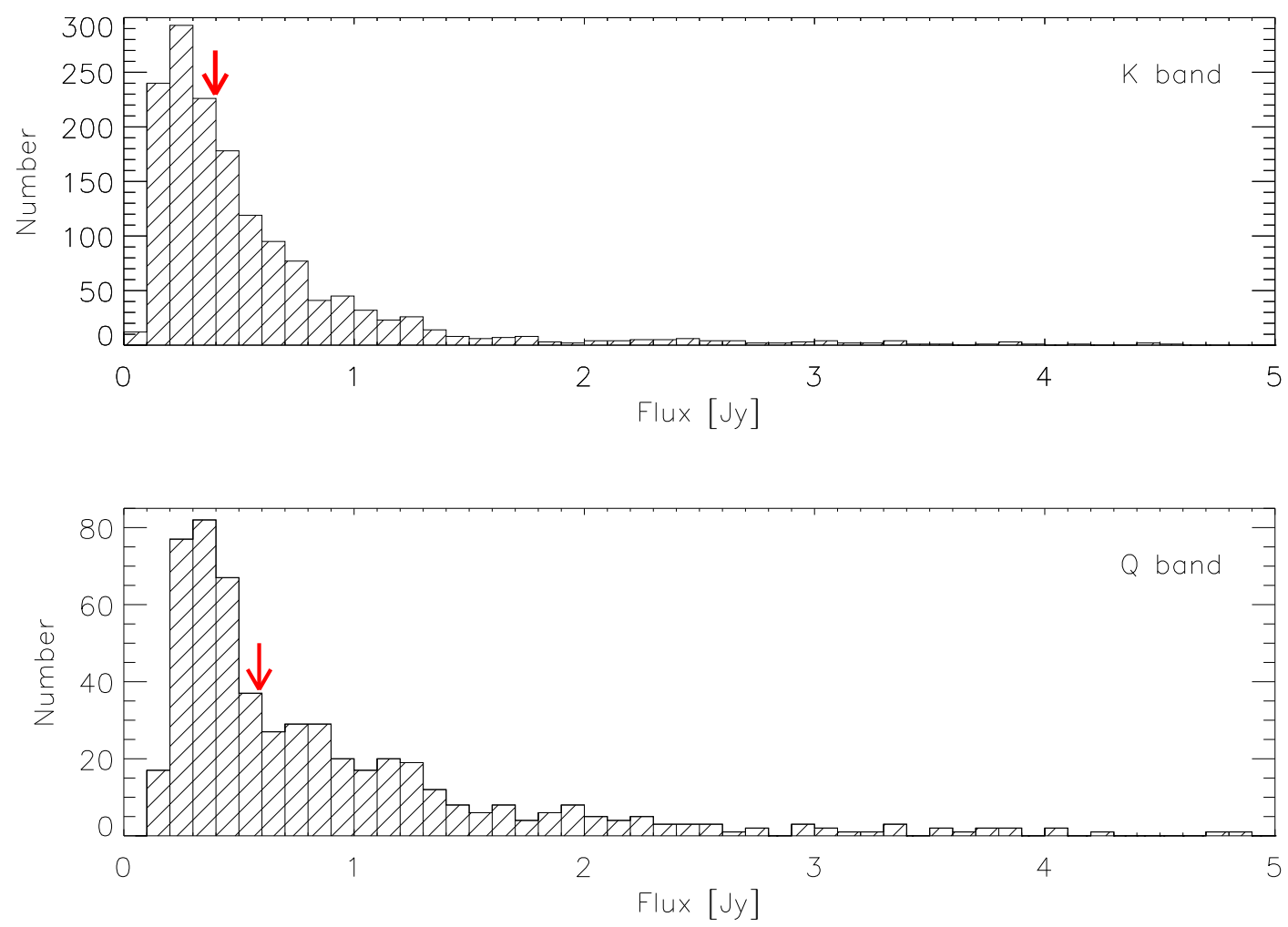

Figure 2. Flux density distributions of measured sources ( $\leq 5 \mathrm{Jy}$ ) in the $\mathrm{K}$ (top) and Q (bottom) bands. Red arrows indicate the median flux densities, 397 and $587 \mathrm{mJy}$ in the $\mathrm{K}$ and $\mathrm{Q}$ bands, respectively, whereas the mean flux densities are 707 and 1,103 mJy, respectively. 

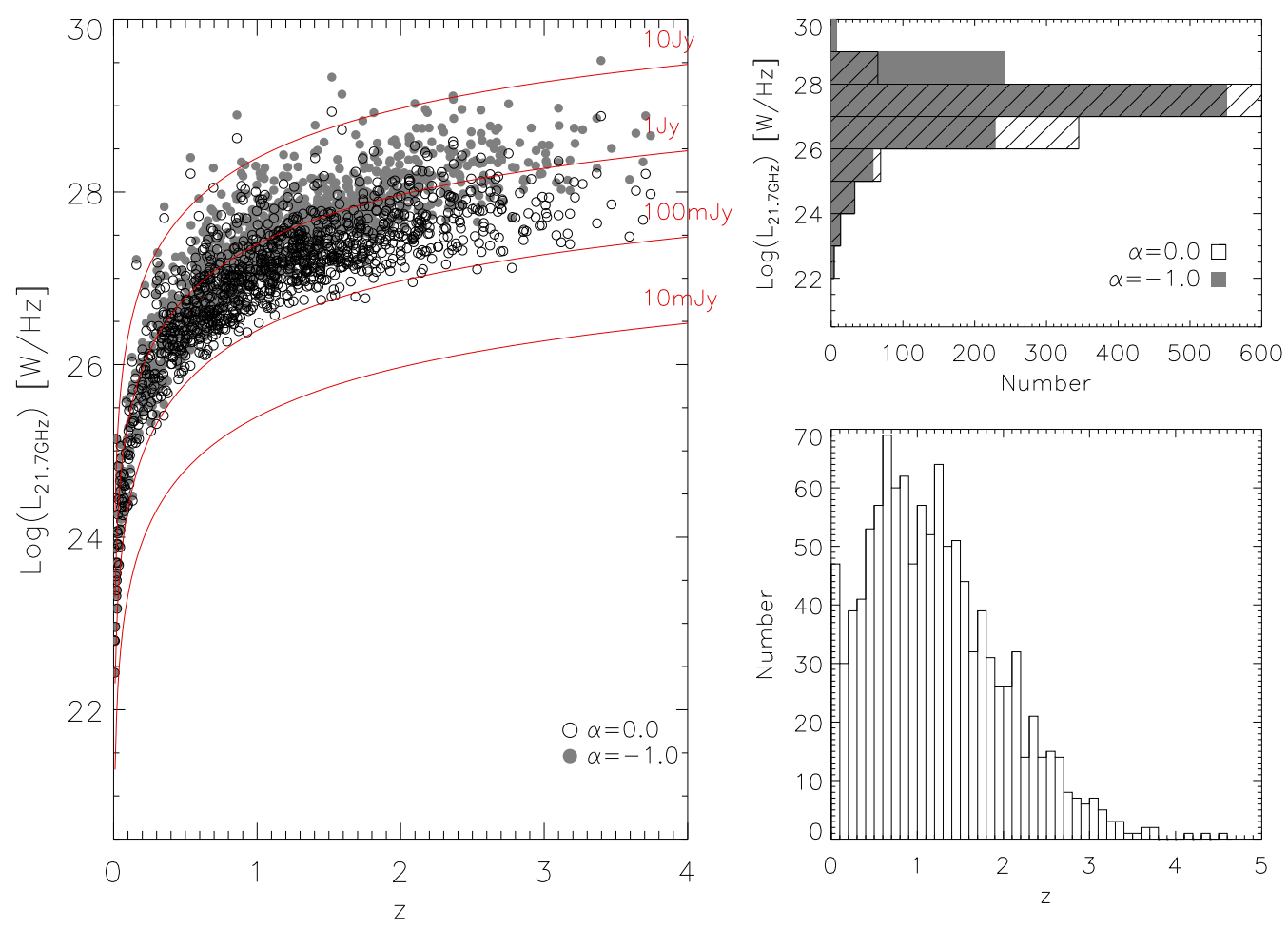

Figure 3. Luminosity distribution of 1137 sources as a function of redshift $(z)$. The $z$ values were taken from the NASA/IPAC Extragalactic Database (NED) and the Sloan Digital Sky Survey (SDSS) DR13. These luminosities are calculated with $H_{0}=$ $73 \mathrm{~km} \mathrm{~s} \mathrm{spc}^{-1}$ and $\Omega_{m}=0.27$ at $21.7 \mathrm{GHz}$, which is the observed frequency, according to the spectral index, $\alpha(-1.0$ : gray filled circles, 0: black circles). The red lines indicates the luminosity distribution at $\alpha=0$ as the criteria for flux densities. Top right: The number of sources according to their luminosities at $\alpha=-1$ and 0 . Bottom right: The number of sources according to $z$. 

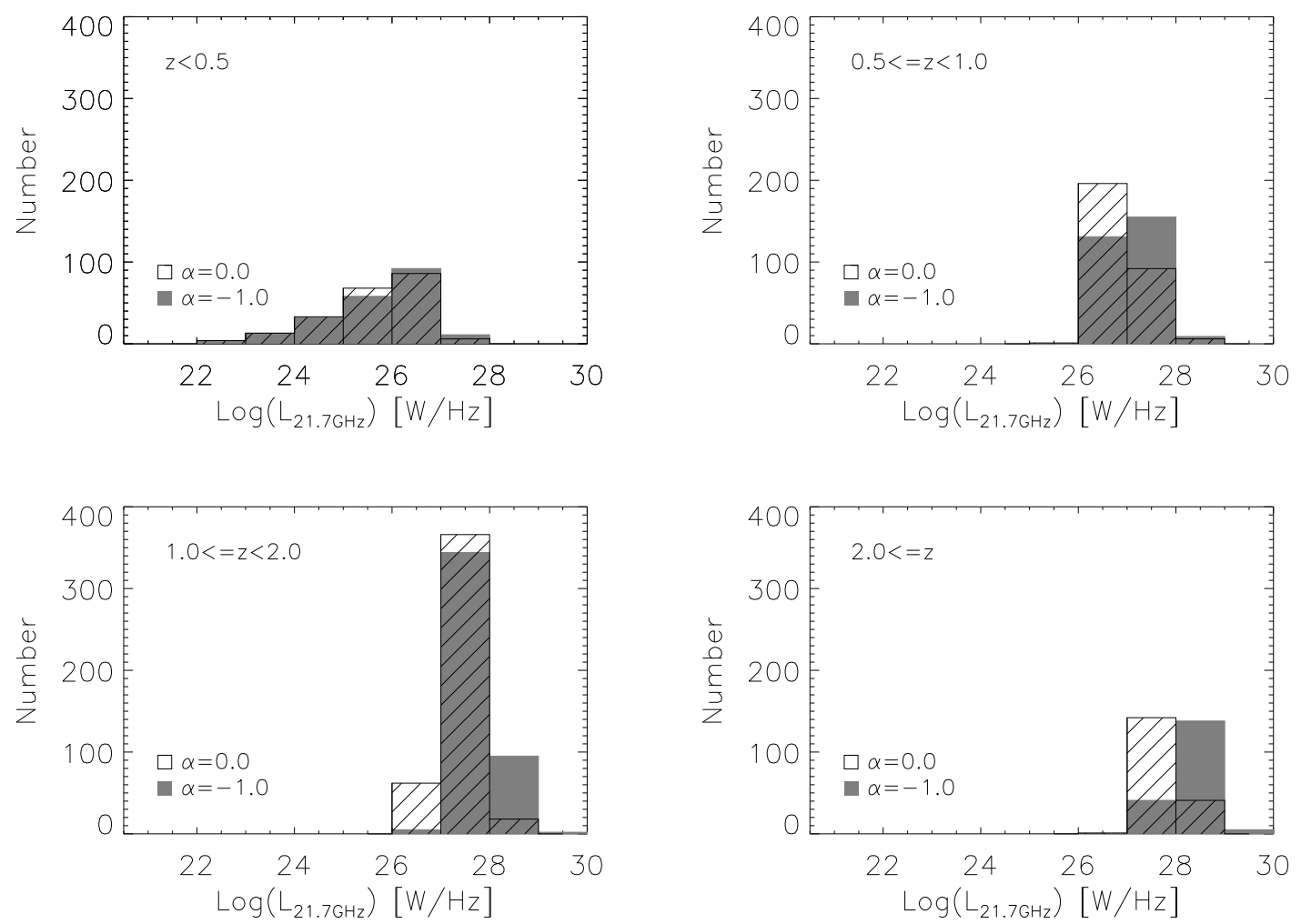

Figure 4. Luminosity distributions in four-redshift $(z)$ bins. Gray filled and black hatched bars stand for the distributions for $\alpha=-1.0$ and 0.0 , respectively. 

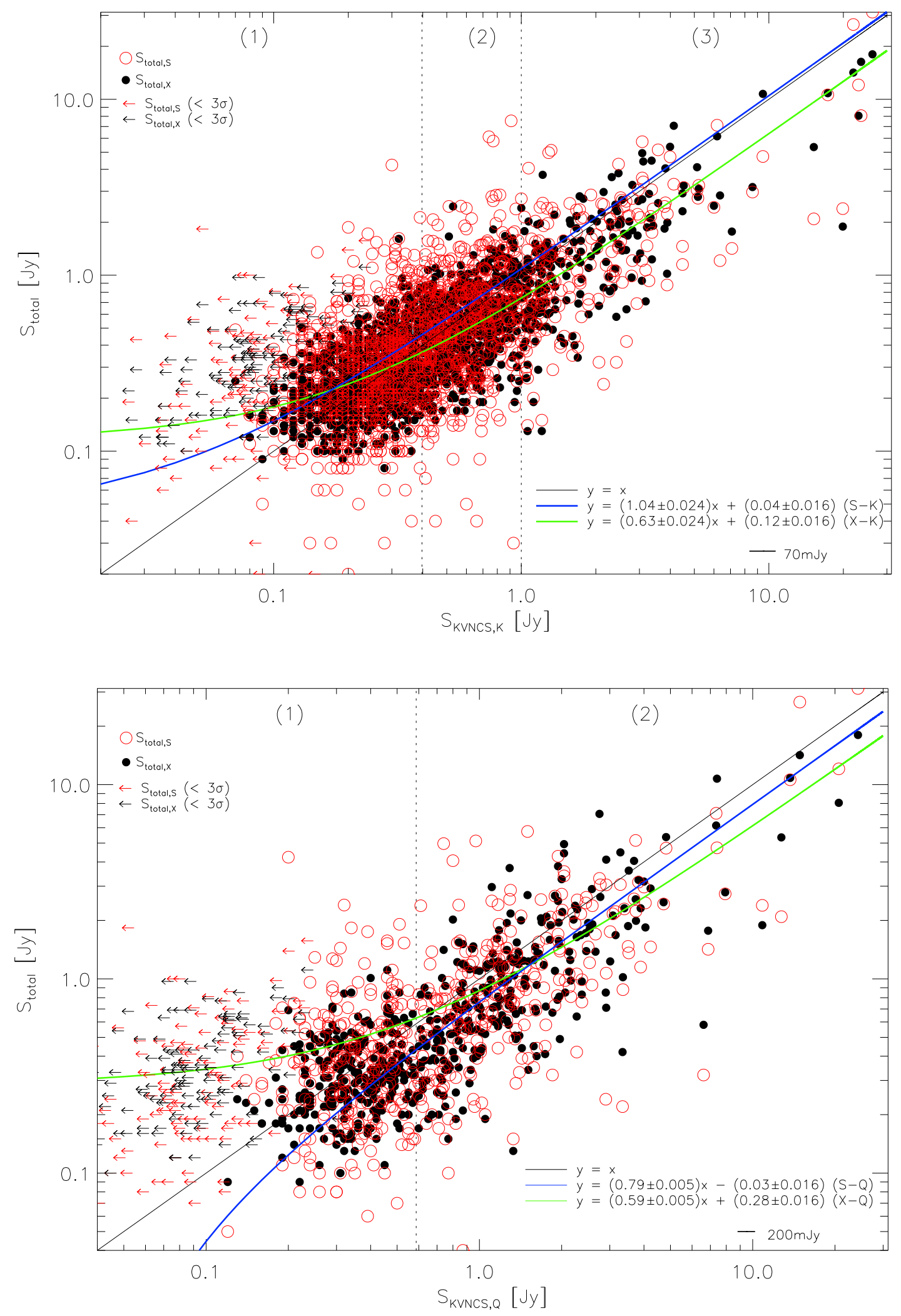

Figure 5. Top: the flux-flux relationship between the VCS $\left(S_{\text {total }}\right)$ in the S (red circle) and X (black dot) bands and the measured flux densities of the KVNCS $\left(S_{K V N C S}\right)$ in the K band. Black and red arrows indicate $1 \sigma$ errors of the sources at less than $3 \sigma$ detection. Black bars on the right and bottom indicate the mean $1 \sigma$ measurement errors ( $70 \mathrm{mJy})$ of the bright sources (over $1 \mathrm{Jy}$ ) in the $\mathrm{K}$ band. Regions (1) and (2) are separated by the median flux densities (the first dotted line) of the KVNCS in the K band. Regions (2) and (3) are separated by 1 Jy (the second dotted line) arbitrarily. The blue (S-K) and green $(\mathrm{X}-\mathrm{K})$ lines are linear fit results obtained using the data in (2), and the black line is a proportional case. Bottom: the flux-flux relationship between the VCS $\left(S_{\text {total }}\right)$ in the S (red circle) and X (black dot) bands and the measured flux densities of the KVNCS $\left(S_{K V N C S}\right)$ in the Q band. Black and red arrows indicate $1 \sigma$ errors of the sources at less than $3 \sigma$ detection. The black bars on the right and bottom indicate the mean $1 \sigma$ measurement errors (200 mJy) of the bright sources over 1 Jy in the $\mathrm{Q}$ band. Region (1) and (2) are separated by the median flux densities (the dotted line) of the KVNCS in the Q band. The 

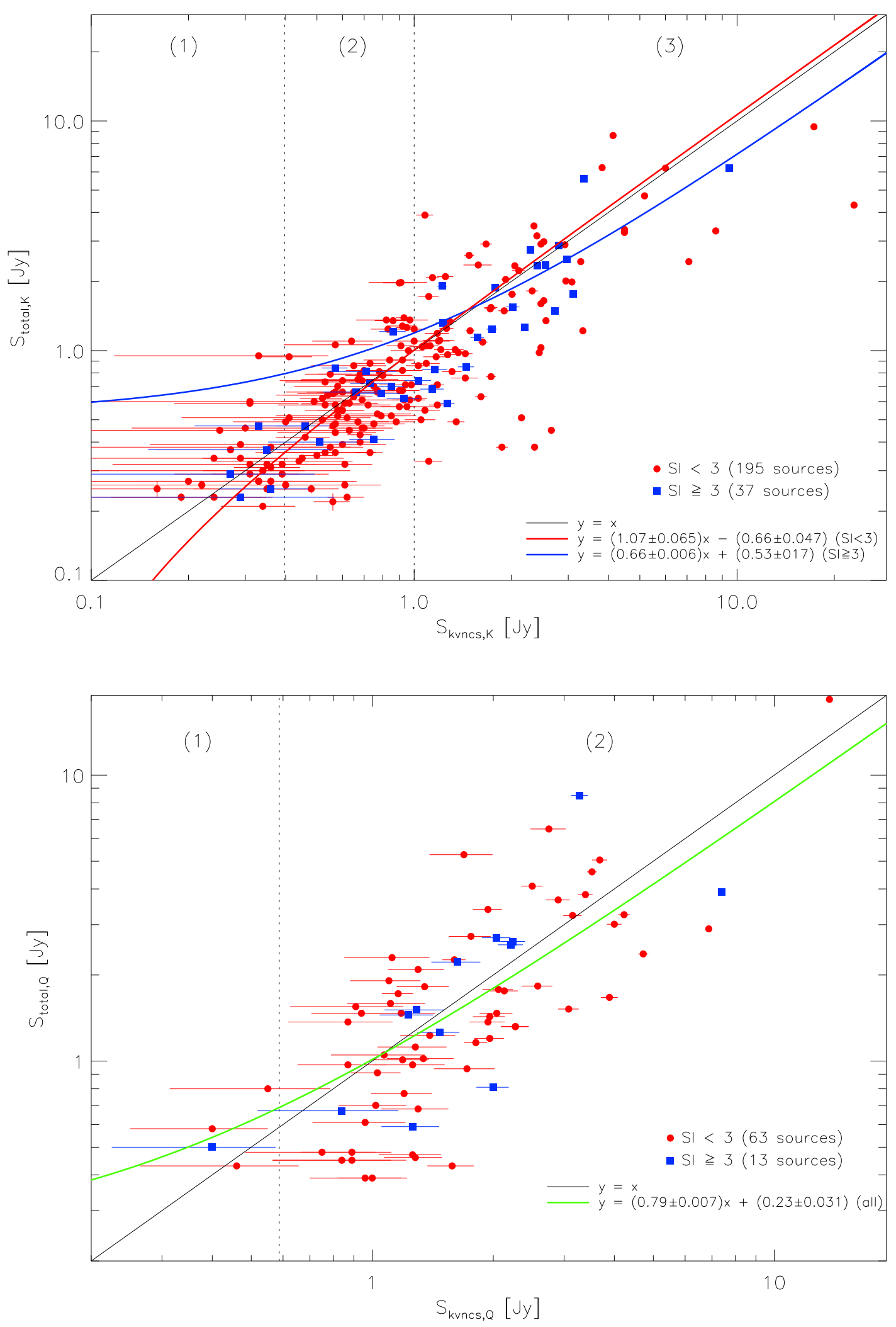

Figure 6. Top: flux-flux relationship of 232 common sources in the K band between single dish observations from the KVNCS and VLBI observations from (Charlot et al. 2010) according to the structure index (SI). The error bar of each source indicates the $1 \sigma$ measurement error. Regions (1) and (2) are separated by the median flux densities (the first dotted line) of the KVNCS in the $\mathrm{K}$ band. Regions (2) and (3) are separated by 1 Jy (the second dotted line) arbitrarily. Red (SI $<3$ ) and blue (SI $\geq 3$ ) lines show the weighted linear fit results obtained using the data in (2) and those from all extended sources, respectively (black line: proportional case). Bottom: flux-flux relationship of 76 common sources in the Q band. The error bar of each source indicates the $1 \sigma$ measurement error. Green line shows the weighted linear fit result obtained using the all data, regardless of the SI (black line: proportional case). 

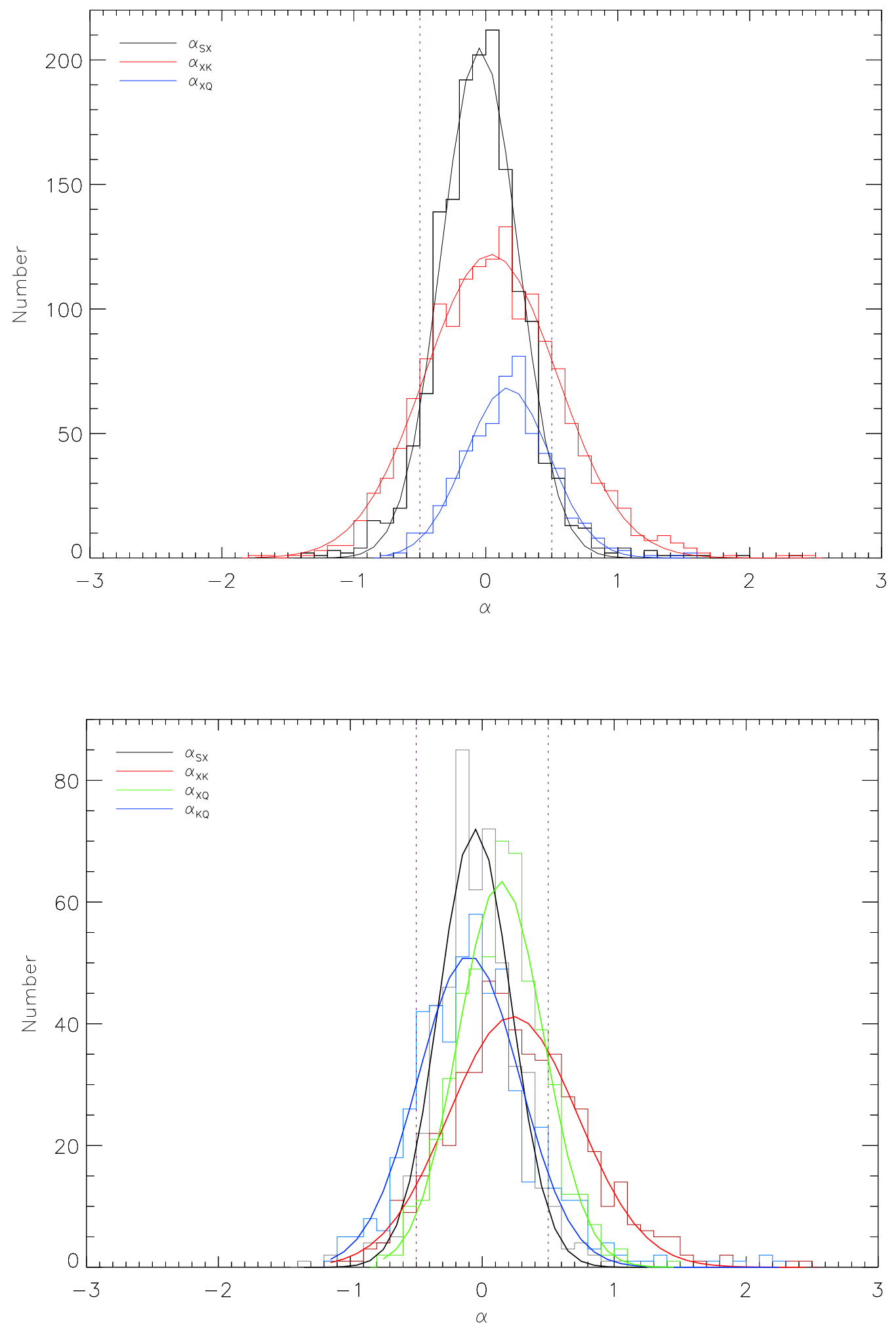

Figure 7. Top: number distributions of $\alpha_{S X}$ (black) and $\alpha_{X K}$ (red) of 1533 sources and $\alpha_{X Q}$ (blue) of 553 sources; black, red, and blue lines are Gaussian fit results; coefficients $=$ (peak, center spectral index, standard deviation): $\alpha_{S X}=(205,-0.045$, $0.290), \alpha_{X K}=(122,0.039,0.499)$, and $\alpha_{X Q}=(68,0.165,0.318)$. Bottom: number distributions of $\alpha_{S X}$ (black), $\alpha_{X K}$ (red), $\alpha_{X Q}$ (green), and $\alpha_{K Q}$ (blue) of 513 sources measured simultaneously in the $\mathrm{K}$ and Q bands; black, red, green, and blue lines are Gaussian fit results; coefficient $=$ (peak, center spectral index, standard deviation): $\alpha_{S X}=(72,-0.055,0.275), \alpha_{X K}=$ $(41,0.23,0.490), \alpha_{X Q}=(63.4,0.14,0.321)$, and $\alpha_{K Q}=(51,-0.101,0.387)$. Vertical purple dotted lines indicate the typical minimum $(\alpha=-0.5)$ and maximum $(\alpha=0.5) \alpha$ of flat spectrum. VLBI total flux densities in the $\mathrm{S}$ and $\mathrm{X}$ bands are taken from the VLBA Calibrator Survey (VCS). 


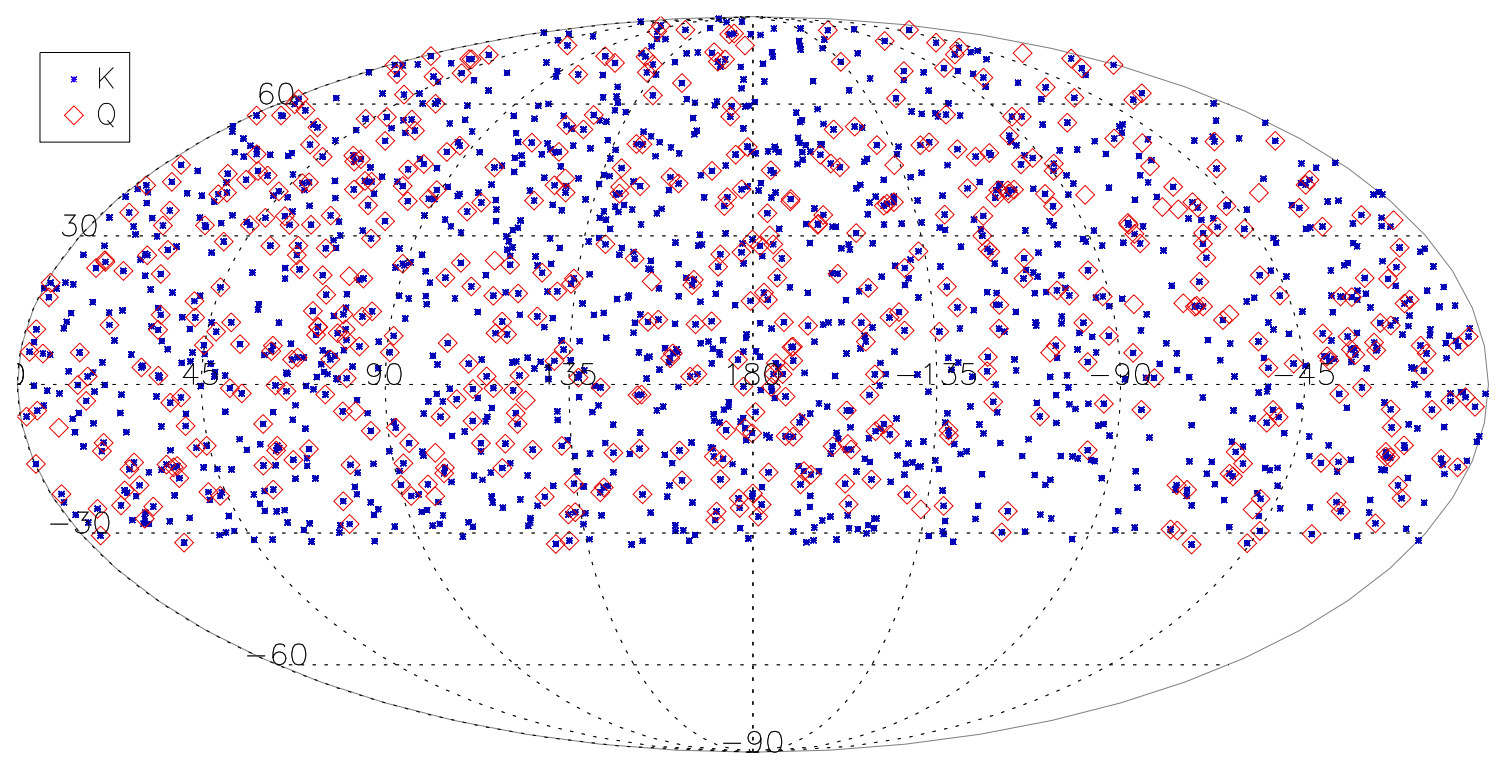

Figure 8. Sky distributions of our samples on the celestial sphere: 1533 (K band, blue asterisks) and 553 (Q-band, red diamonds) sources (same as Figure 1 in (Lee et al. 2012)). The lower limit in declination is $-32.5^{\circ}$. (Abscissa: right ascension $\left[^{\circ}\right]$, ordinate: declination $\left.\left[^{\circ}\right]\right)$. 


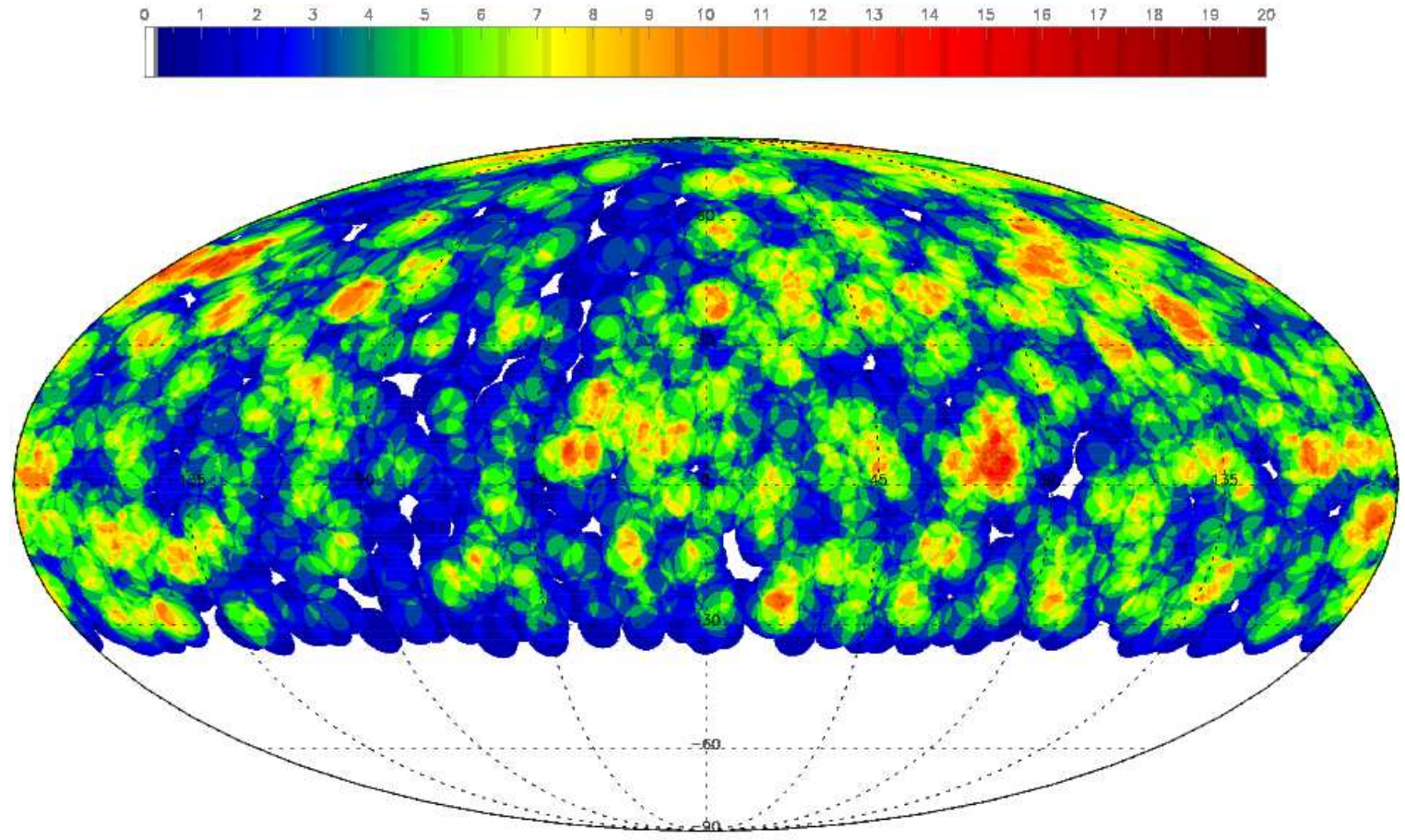

Figure 9. Sky coverage of 1533 sources by assuming a spatial coherence of $5^{\circ}$ (radius of circles in Figure 1). Colored legend shows the values represented by overlapping circles. More than two sources are overlapped (89\%); a single source (10\%); no source $(1 \%)$ is indicated by white regions. Mollwide equal-area projection technique was used (abscissa: right ascension [ ${ }^{\circ}$, ordinate: declination $\left[^{\circ}\right]$ ) 
Table 1. Observational information

\begin{tabular}{|c|c|c|c|c|c|c|c|}
\hline Date & $\begin{array}{l}\text { Observation } \\
\text { code }\end{array}$ & $\begin{array}{l}\text { Number of } \\
\text { sources }[\#]\end{array}$ & Telescope* & $\begin{array}{c}\text { On-source } \\
\text { integration time }[\mathrm{s}]\end{array}$ & $\begin{array}{c}\text { Tsys }^{\dagger} \\
{[\mathrm{K}]}\end{array}$ & $\tau_{0}^{\dagger \dagger}$ & $\begin{array}{r}\text { Conversion } \\
\text { factor }[\mathrm{Jy} / \mathrm{K}]\end{array}$ \\
\hline Dec. 2009 & KVNCS1.0.1 & $\cdots$ & KYS & $40(\mathrm{~K}), 20(\mathrm{Q})$ & $96.2(\mathrm{~K}), 181.7(\mathrm{Q})$ & $0.053(\mathrm{~K}), 0.097(\mathrm{Q})$ & $12.95(\mathrm{~K}), 12.99(\mathrm{Q})$ \\
\hline Feb. 2010 & KVNCS1.1.1 & $\cdots$ & KYS & $40(\mathrm{~K}), 20(\mathrm{Q})$ & $74.3(\mathrm{~K}), 157.4(\mathrm{Q})$ & $0.027(\mathrm{~K}), 0.088(\mathrm{Q})$ & $12.78(\mathrm{~K}), 11.82(\mathrm{Q})$ \\
\hline Mar. 2010 & KVNCS1.1.2 & $\cdots$ & KYS & $40(\mathrm{~K}), 20(\mathrm{Q})$ & $97.0(\mathrm{~K}), 168.9(\mathrm{Q})$ & $0.068(\mathrm{~K}), 0.104(\mathrm{Q})$ & $13.11(\mathrm{~K}), 12.88(\mathrm{Q})$ \\
\hline May 2010 & KVNCS1.1.3 & $595 \AA$ & KYS & $40(\mathrm{~K}), 20(\mathrm{Q})$ & $220.4(\mathrm{~K}), 397.1(\mathrm{Q})$ & $0.103(\mathrm{~K}), 0.111(\mathrm{Q})$ & $12.97(\mathrm{~K}), 12.45(\mathrm{Q})$ \\
\hline June 2010 & KVNCS1.2.1 & 396 & KYS & $80(\mathrm{~K}), 40(\mathrm{Q})$ & $124.7(\mathrm{~K}), 184.0(\mathrm{Q})$ & $0.111(\mathrm{~K}), 0.116(\mathrm{Q})$ & $12.97(\mathrm{~K}), 12.45(\mathrm{Q})$ \\
\hline Dec. 2010 & KVNCS1.2.2 & 670 & KUS & $80(\mathrm{~K}), 40(\mathrm{Q})$ & $95.8(\mathrm{~K}), 119.4(\mathrm{Q})$ & $0.051(\mathrm{~K}), 0.086(\mathrm{Q})$ & $13.67(\mathrm{~K}), 15.33(\mathrm{Q})$ \\
\hline Jan. 2011 & KVNCS1.3.1 & 573 & KUS & $160(\mathrm{~K}), 80(\mathrm{Q})$ & $82.9(\mathrm{~K}), 137.5(\mathrm{Q})$ & $0.034(\mathrm{~K}), 0.092(\mathrm{Q})$ & $13.85(\mathrm{~K}), 16.24(\mathrm{Q})$ \\
\hline Mar. 2011 & KVNCS1.3.2 & 182 & KYS & $160(\mathrm{~K}), 80(\mathrm{Q})$ & $88.3(\mathrm{~K}), 180.2(\mathrm{Q})$ & $0.042(\mathrm{~K}), 0.091(\mathrm{Q})$ & $12.99(\mathrm{~K}), 13.51(\mathrm{Q})$ \\
\hline
\end{tabular}

* KYS: KVN Yonsei Radio Observatory, KUS: KVN Ulsan Radio Observatory

$\dagger$ Mean system temperature of each observation

$\dagger$ Mean zenith optical depth of each observation

$\S$ Total number of sources from KVNCS1.0.1 to KVNCS1.1.3 is 595. 
Table 2. Examples from the KVNCS Catalog

\begin{tabular}{|c|c|c|c|c|c|c|c|c|}
\hline $\begin{array}{c}\text { IAU name } \\
(\mathrm{J} 2000) \\
(1) \\
\end{array}$ & IVS name & $\begin{array}{c}\text { R.A. } \\
\text { [hh:mm:ss] } \\
(3) \\
\end{array}$ & $\begin{array}{c}\text { Dec. } \\
{\left[{ }^{\circ}:^{\prime}:^{\prime \prime}\right]} \\
(4) \\
\end{array}$ & Obs. code & $\begin{array}{c}\text { Flux density }( \pm 1 \sigma) \\
{[\mathrm{Jy}]} \\
(6)\end{array}$ & Obs. code & $\begin{array}{c}\text { Flux density }( \pm 1 \sigma) \\
{[\mathrm{Jy}]} \\
(8)\end{array}$ & Redshift (z) \\
\hline $\mathrm{J} 0007+5706$ & $0005+568$ & $00: 07: 48.47$ & $57: 06: 10.44$ & KVNCS1.3.1 & $0.34( \pm 0.04)$ & KVNCS1.3.1 & $0.23( \pm 0.05)$ & $\cdots$ \\
\hline J0008-2339 & $0005-239$ & 00:08:00.37 & $-23: 39: 18.00$ & KVNCS1.3.1 & $0.16( \pm 0.03)$ & $\cdots$ & $\ldots$ & 1.412 \\
\hline J0008+6837 & $0005+683$ & $00: 08: 33.47$ & $68: 37: 22.08$ & KVNCS1.3.1 & $0.21( \pm 0.02)$ & $\ldots$ & $\ldots$ & $\cdots$ \\
\hline $\mathrm{J} 0009+0628$ & $0006+061$ & 00:09:03.93 & $06: 28: 21.25$ & KVNCS1.3.1 & $0.22( \pm 0.03)$ & KVNCS1.3.1 & $0.33( \pm 0.05)$ & $\cdots$ \\
\hline J0009+4001 & $0006+397$ & 00:09:04.17 & 40:01:46.56 & KVNCS1.3.2 & $0.29( \pm 0.04)$ & $\cdots$ & $\ldots$ & 1.830 \\
\hline $\mathrm{J} 0010+1058$ & IIIZW2 & $00: 10: 31.00$ & $10: 58: 29.64$ & KVNCS1.0.1 & $2.15( \pm 0.12)$ & KVNCS1.0.1 & $2.90( \pm 0.58)$ & 0.089 \\
\hline $\mathrm{J} 0010+1724$ & $0007+171$ & $00: 10: 33.99$ & $17: 24: 18.72$ & KVNCS1.3.1 & $0.50( \pm 0.04)$ & KVNCS1.3.1 & $0.46( \pm 0.07)$ & 1.601 \\
\hline J0010-2157 & 0008-222 & $00: 10: 53.65$ & $-21: 57: 04.32$ & KVNCS1.3.1 & $0.41( \pm 0.03)$ & KVNCS1.3.1 & $0.45( \pm 0.07)$ & $\ldots$ \\
\hline J0011-2612 & $0008-264$ & $00: 11: 01.25$ & $-26: 12: 33.48$ & KVNCS1.2.2 & $0.45( \pm 0.05)$ & $\ldots$ & $\ldots$ & 1.096 \\
\hline $\mathrm{J} 0011+7045$ & $0008+704$ & $00: 11: 31.90$ & $70: 45: 31.68$ & KVNCS1.3.1 & $0.47( \pm 0.05)$ & KVNCS1.3.1 & $0.44( \pm 0.06)$ & $\cdots$ \\
\hline
\end{tabular}

Note-(1) IAU name (J2000), (2) IVS names, (3) and (4) are the right ascension and declination taken from the VCS1-5, respectively, (5) and (6) are an observation code and the source flux density with $1 \sigma$ error in the blanket in the K band, respectively, (7) and (8) are the same as (5) and (6) only in the Q band, (9) redshifts $(z)$ of 1137 sources come from NASA/IPAC Extragalactic Database (NED) and Sloan Digital Sky Survey (SDSS) DR13. Full KVNCS catalog is given in the appendix. 
Table 3. Statistics of $\alpha$ for 1533 sources in the $\mathrm{K}$ band, 553 sources in the Q band, and 513 sources measured simultaneously in the $\mathrm{K}$ and Q bands

\begin{tabular}{|c|c|c|c|c|c|c|}
\hline \multirow[b]{2}{*}{ Spectral index } & \multicolumn{3}{|c|}{$1533(\mathrm{~K}) \& 553(\mathrm{Q})$ sources } & \multicolumn{3}{|c|}{513 sources } \\
\hline & $\begin{array}{c}\text { Steep } \\
{[\%]}\end{array}$ & $\begin{array}{r}\text { Flat } \\
{[\%]}\end{array}$ & $\begin{array}{c}\text { Invert } \\
{[\%]}\end{array}$ & $\begin{array}{c}\text { Steep } \\
{[\%]}\end{array}$ & $\begin{array}{r}\text { Flat } \\
{[\%]}\end{array}$ & $\begin{array}{c}\text { Invert } \\
{[\%]}\end{array}$ \\
\hline$\alpha_{S X}$ & 6.9 & 88.5 & 4.6 & 6.4 & 89.5 & 4.1 \\
\hline$\alpha_{X K}$ & 12.9 & 68.2 & 19.9 & 5.8 & 62.6 & 31.6 \\
\hline$\alpha_{X Q}$ & 2.3 & 82.3 & 15.4 & 2.7 & 84.2 & 13.0 \\
\hline$\alpha_{K Q}$ & $\ldots$ & $\ldots$ & $\ldots$ & 13.5 & 76.2 & 10.3 \\
\hline
\end{tabular}




\section{APPENDIX}

Table 2. The KVNCS catalog

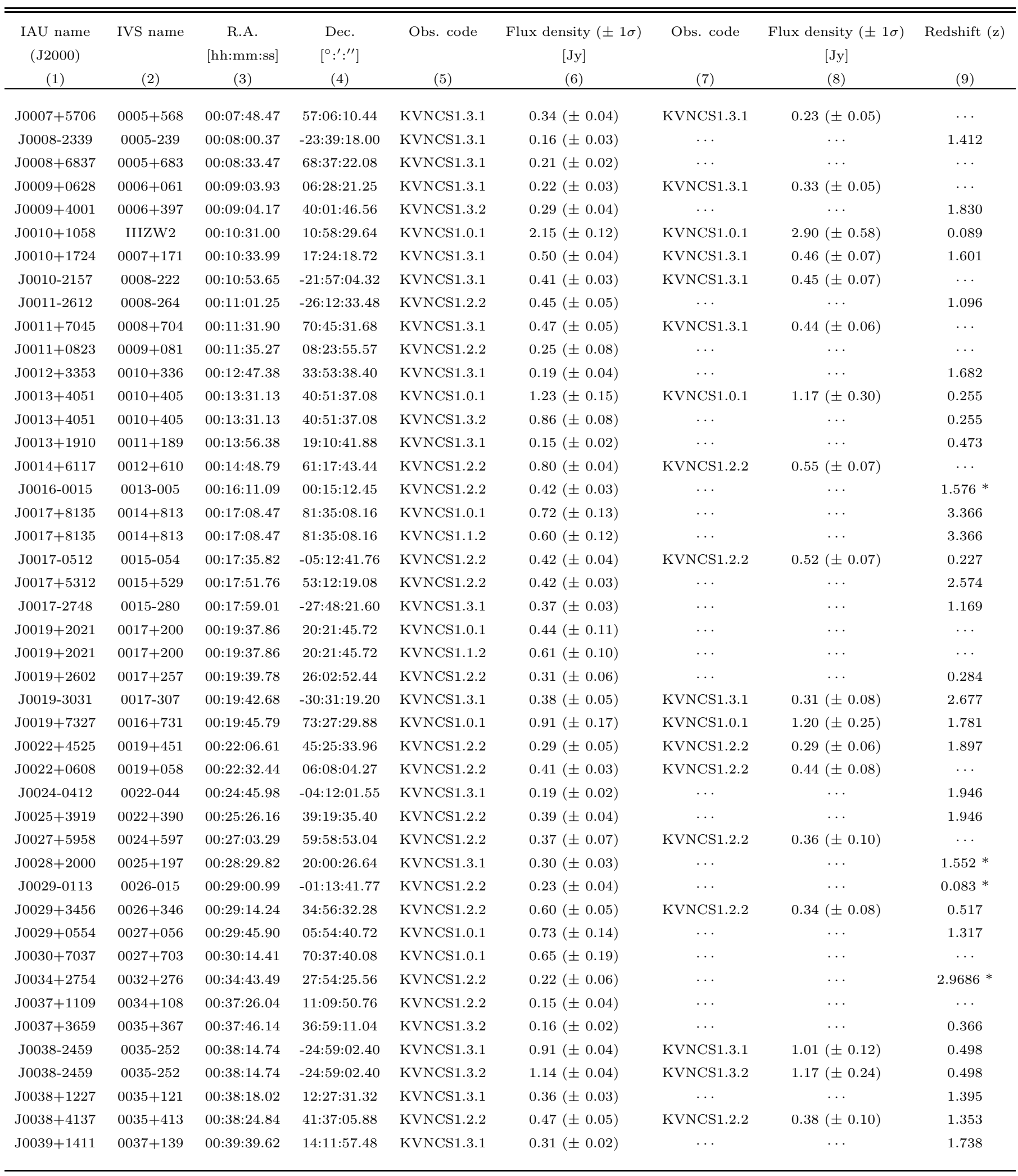


Table 2 (continued)

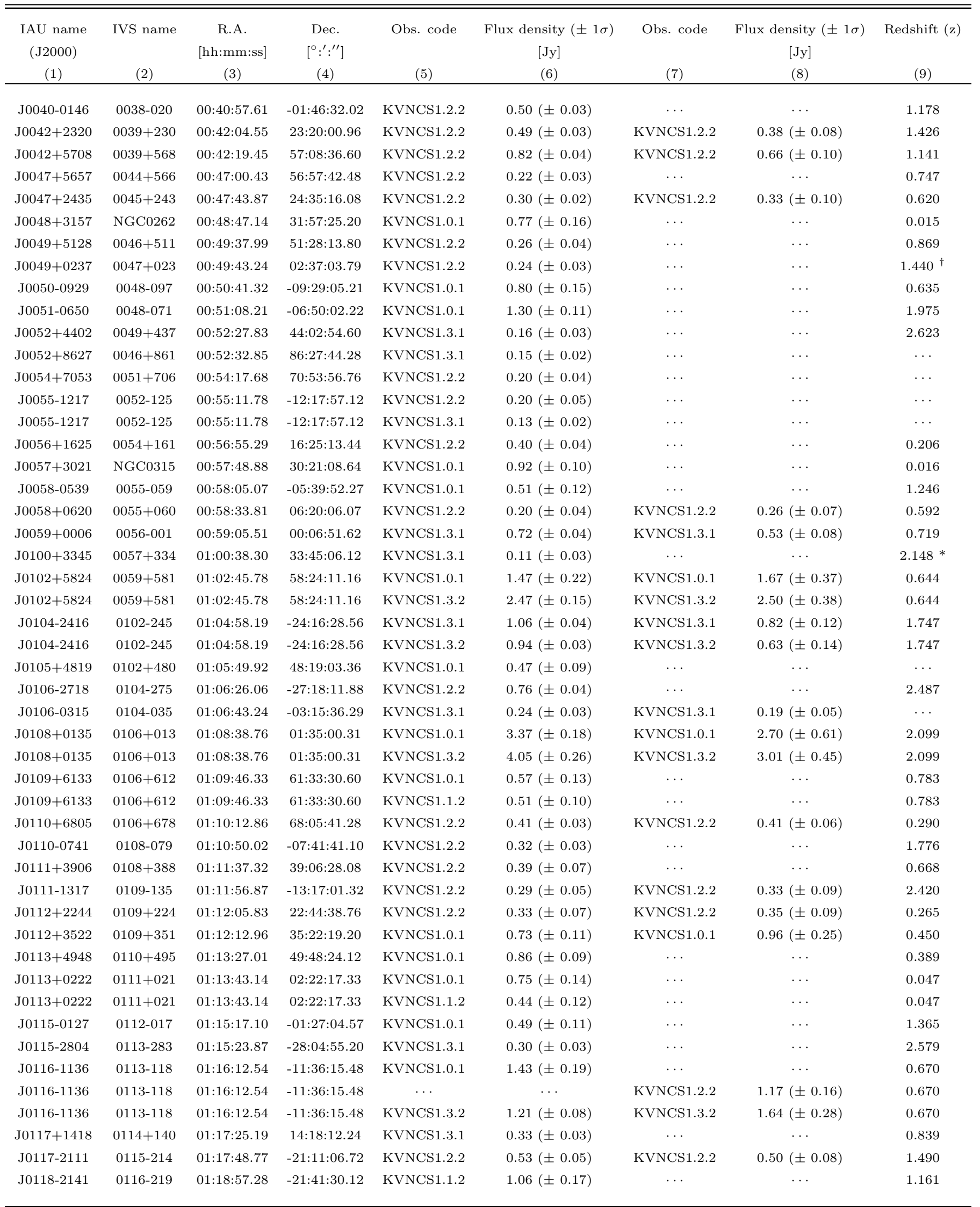


Table 2 (continued)

\begin{tabular}{|c|c|c|c|c|c|c|c|c|}
\hline $\begin{array}{l}\text { IAU name } \\
(\mathrm{J} 2000) \\
(1) \\
\end{array}$ & IVS name & $\begin{array}{c}\text { R.A. } \\
\text { [hh:mm:ss] } \\
(3) \\
\end{array}$ & $\begin{array}{c}\text { Dec. } \\
{\left[{ }^{\circ}:^{\prime}:^{\prime \prime}\right]} \\
(4)\end{array}$ & $\begin{array}{l}\text { Obs. code } \\
\qquad(5) \\
\end{array}$ & $\begin{array}{c}\text { Flux density }( \pm 1 \sigma) \\
{[\mathrm{Jy}]} \\
(6)\end{array}$ & Obs. code & $\begin{array}{c}\text { Flux density }( \pm 1 \sigma) \\
{[\mathrm{Jy}]} \\
(8)\end{array}$ & Redshift (z) \\
\hline $\mathrm{J} 0119+3210$ & $0116+319$ & 01:19:35.00 & $32: 10: 50.16$ & KVNCS1.3.2 & $0.56( \pm 0.03)$ & KVNCS1.3.2 & $0.31( \pm 0.09)$ & 0.060 \\
\hline J0120-2701 & $0118-272$ & $01: 20: 31.67$ & $-27: 01: 24.60$ & KVNCS1.3.1 & $0.72( \pm 0.04)$ & KVNCS1.3.1 & $0.72( \pm 0.09)$ & 0.559 \\
\hline J0121-2806 & $0118-283$ & $01: 21: 00.76$ & $-28: 06: 22.32$ & KVNCS1.3.1 & $0.13( \pm 0.02)$ & $\cdots$ & $\ldots$ & 3.119 \\
\hline $\mathrm{J} 0121+1149$ & $0119+115$ & $01: 21: 41.58$ & $11: 49: 50.52$ & KVNCS1.0.1 & $2.20( \pm 0.13)$ & KVNCS1.0.1 & $1.83( \pm 0.45)$ & $0.571 *$ \\
\hline $\mathrm{J} 0121+1149$ & $0119+115$ & $01: 21: 41.58$ & $11: 49: 50.52$ & KVNCS1.3.2 & $1.67( \pm 0.14)$ & KVNCS1.3.2 & $1.23( \pm 0.23)$ & $0.571 *$ \\
\hline $\mathrm{J} 0121+0422$ & $0119+041$ & $01: 21: 56.84$ & $04: 22: 24.74$ & KVNCS1.0.1 & $0.90( \pm 0.12)$ & $\cdots$ & $\cdots$ & 0.637 \\
\hline $\mathrm{J} 0122+2502$ & $0119+247$ & $01: 22: 38.82$ & $25: 02: 31.92$ & KVNCS1.0.1 & $0.56( \pm 0.14)$ & $\cdots$ & $\cdots$ & 2.025 \\
\hline J0122+2954 & $0119+296$ & $01: 22: 45.44$ & $29: 54: 12.60$ & $\mathrm{KVNCS} 1.2 .2$ & $0.24( \pm 0.06)$ & $\cdots$ & $\cdots$ & $\cdots$ \\
\hline $\mathrm{J} 0123+2615$ & $0120+259$ & $01: 23: 43.04$ & $26: 15: 22.32$ & KVNCS1.3.1 & $0.23( \pm 0.04)$ & $\cdots$ & $\cdots$ & 0.849 \\
\hline $\mathrm{J} 0125+4718$ & $0122+470$ & $01: 25: 07.72$ & $47: 18: 03.24$ & $\mathrm{KVNCS} 1.2 .2$ & $0.18( \pm 0.04)$ & $\cdots$ & $\cdots$ & $\cdots$ \\
\hline J0125-2549 & $0122-260$ & $01: 25: 18.84$ & $-25: 49: 04.44$ & KVNCS1.3.1 & $0.17( \pm 0.04)$ & $\cdots$ & $\cdots$ & $\cdots$ \\
\hline J0125-0005 & 0122-003 & $01: 25: 28.85$ & $00: 05: 55.93$ & KVNCS1.0.1 & $1.14( \pm 0.11)$ & $\cdots$ & $\cdots$ & 1.075 \\
\hline J0126-2222 & $0123-226$ & $01: 26: 15.00$ & $-22: 22: 33.60$ & KVNCS1.2.2 & $0.29( \pm 0.03)$ & $\ldots$ & $\cdots$ & 0.720 \\
\hline J0126+2559 & $0123+257$ & $01: 26: 42.79$ & $25: 59: 01.32$ & KVNCS1.2.2 & $0.69( \pm 0.05)$ & KVNCS1.2.2 & $0.49( \pm 0.06)$ & $2.368 *$ \\
\hline J0128+4901 & $0125+487$ & 01:28:08.08 & 49:01:05.88 & KVNCS1.2.2 & $0.43( \pm 0.02)$ & $\cdots$ & $\ldots$ & 0.067 \\
\hline J0129+1446 & $0127+145$ & $01: 29: 55.36$ & $14: 46: 48.00$ & KVNCS1.2.2 & $0.32( \pm 0.07)$ & $\cdots$ & $\cdots$ & 1.626 \\
\hline $\mathrm{J} 0130+0842$ & $0127+084$ & $01: 30: 27.65$ & $08: 42: 46.19$ & KVNCS1.2.2 & $0.20( \pm 0.05)$ & $\cdots$ & $\cdots$ & 0.725 \\
\hline J0131+3834 & $0128+383$ & $01: 31: 26.72$ & $38: 34: 39.36$ & KVNCS1.2.2 & $0.27( \pm 0.04)$ & $\cdots$ & $\cdots$ & 1.277 \\
\hline J0132-1654 & 0130-171 & $01: 32: 43.48$ & $-16: 54: 48.60$ & KVNCS1.1.2 & $1.40( \pm 0.16)$ & KVNCS1.1.2 & $1.41( \pm 0.43)$ & 1.020 \\
\hline J0132-1654 & $0130-171$ & $01: 32: 43.48$ & $-16: 54: 48.60$ & KVNCS1.3.2 & $3.18( \pm 0.18)$ & KVNCS1.3.2 & $2.91( \pm 0.43)$ & 1.020 \\
\hline $\mathrm{J} 0132+4325$ & $0129+431$ & $01: 32: 44.12$ & $43: 25: 32.52$ & KVNCS1.3.1 & $0.48( \pm 0.02)$ & KVNCS1.3.1 & $0.39( \pm 0.06)$ & $\cdots$ \\
\hline J0135-2008 & 0133-204 & $01: 35: 37.50$ & $-20: 08: 45.96$ & $\mathrm{KVNCS} 1.2 .2$ & $0.26( \pm 0.03)$ & $\mathrm{KVNCS} 1.2 .2$ & $0.34( \pm 0.08)$ & 1.144 \\
\hline J0136+4751 & $0133+476$ & $01: 36: 58.61$ & $47: 51: 29.16$ & KVNCS1.0.1 & $4.13( \pm 0.31)$ & KVNCS1.0.1 & $3.74( \pm 0.59)$ & 0.859 \\
\hline $\mathrm{J} 0136+4751$ & $0133+476$ & $01: 36: 58.61$ & $47: 51: 29.16$ & KVNCS1.3.2 & $2.40( \pm 0.14)$ & KVNCS1.3.2 & $1.94( \pm 0.31)$ & 0.859 \\
\hline $\mathrm{J} 0137+2145$ & $0134+215$ & $01: 37: 15.64$ & $21: 45: 44.28$ & $\mathrm{KVNCS} 1.2 .2$ & $0.19( \pm 0.04)$ & $\ldots$ & $\ldots$ & $\cdots$ \\
\hline J0137-2430 & $0135-247$ & $01: 37: 38.35$ & $-24: 30: 54.00$ & KVNCS1.1.2 & $2.43( \pm 0.21)$ & KVNCS1.1.2 & $2.37( \pm 0.65)$ & 0.838 \\
\hline J0137-2430 & $0135-247$ & $01: 37: 38.35$ & $-24: 30: 54.00$ & KVNCS1.3.2 & $1.26( \pm 0.09)$ & $\cdots$ & $\ldots$ & 0.838 \\
\hline J0138-0540 & 0136-059 & $01: 38: 51.86$ & $-05: 40: 08.26$ & $\mathrm{KVNCS} 1.2 .2$ & $0.17( \pm 0.03)$ & $\ldots$ & $\ldots$ & 2.004 \\
\hline J0140-1532 & $0137-158$ & 01:40:04.44 & $-15: 32: 55.68$ & KVNCS1.2.2 & $0.29( \pm 0.03)$ & KVNCS1.2.2 & $0.31( \pm 0.10)$ & 1.669 \\
\hline J0141-0928 & 0138-097 & $01: 41: 25.84$ & $-09: 28: 43.68$ & KVNCS1.0.1 & $0.94( \pm 0.13)$ & $\cdots$ & $\cdots$ & 0.733 \\
\hline J0141-0928 & 0138-097 & $01: 41: 25.84$ & $-09: 28: 43.68$ & KVNCS1.1.2 & $1.11( \pm 0.12)$ & $\cdots$ & $\cdots$ & 0.733 \\
\hline J0141-0202 & 0139-022 & $01: 41: 33.79$ & $-02: 02: 21.55$ & KVNCS1.2.2 & $0.21( \pm 0.04)$ & $\cdots$ & $\cdots$ & 1.281 \\
\hline $\mathrm{J} 0143+4129$ & $0140+412$ & $01: 43: 03.18$ & $41: 29: 20.40$ & KVNCS1.2.2 & $0.19( \pm 0.03)$ & $\ldots$ & $\ldots$ & 0.824 \\
\hline J0143-3200 & $0140-322$ & $01: 43: 10.13$ & $-32: 00: 56.52$ & KVNCS1.3.1 & $0.53( \pm 0.04)$ & KVNCS1.3.1 & $0.55( \pm 0.12)$ & 0.375 \\
\hline J0143-3200 & 0140-322 & $01: 43: 10.13$ & $-32: 00: 56.52$ & KVNCS1.3.2 & $0.54( \pm 0.04)$ & KVNCS1.3.2 & $0.65( \pm 0.16)$ & 0.375 \\
\hline $\mathrm{J} 0144+2705$ & $0141+268$ & $01: 44: 33.54$ & $27: 05: 03.12$ & KVNCS1.2.2 & $0.30( \pm 0.07)$ & KVNCS1.2.2 & $0.38( \pm 0.07)$ & $\cdots$ \\
\hline J0145-2733 & $0142-278$ & $01: 45: 03.38$ & $-27: 33: 34.20$ & KVNCS1.1.2 & $0.94( \pm 0.18)$ & $\cdots$ & $\cdots$ & 1.155 \\
\hline J0148+3854 & $0145+386$ & $01: 48: 24.37$ & $38: 54: 05.40$ & KVNCS1.2.2 & $0.26( \pm 0.03)$ & KVNCS1.2.2 & $0.35( \pm 0.09)$ & 1.442 \\
\hline J0149+0555 & $0146+056$ & $01: 49: 22.37$ & $05: 55: 53.58$ & KVNCS1.0.1 & $0.49( \pm 0.14)$ & $\cdots$ & $\cdots$ & $2.348 *$ \\
\hline J0149+0555 & $0146+056$ & $01: 49: 22.37$ & $05: 55: 53.58$ & KVNCS1.1.2 & $0.71( \pm 0.12)$ & $\cdots$ & $\cdots$ & $2.348 *$ \\
\hline J0149+1857 & $0147+187$ & $01: 49: 49.73$ & $18: 57: 20.52$ & KVNCS1.2.2 & $0.30( \pm 0.04)$ & $\cdots$ & $\cdots$ & 0.584 \\
\hline J0151-1732 & $0148-177$ & 01:51:06.08 & $-17: 32: 44.88$ & $\mathrm{KVNCS} 1.2 .2$ & $0.36( \pm 0.05)$ & $\cdots$ & $\cdots$ & 0.698 \\
\hline $\mathrm{J} 0151+2744$ & $0148+274$ & $01: 51: 27.14$ & $27: 44: 41.64$ & KVNCS1.2.2 & $0.41( \pm 0.04)$ & $\cdots$ & $\cdots$ & 1.260 \\
\hline $\mathrm{J} 0151+5454$ & $0148+546$ & $01: 51: 36.29$ & $54: 54: 37.80$ & KVNCS1.3.1 & $0.18( \pm 0.03)$ & $\cdots$ & $\cdots$ & $\cdots$ \\
\hline J0152+3716 & $0149+370$ & $01: 52: 12.22$ & $37: 16: 05.52$ & KVNCS1.2.2 & $0.28( \pm 0.03)$ & $\ldots$ & $\cdots$ & $\cdots$ \\
\hline J0152+2207 & $0149+218$ & $01: 52: 18.05$ & $22: 07: 07.68$ & KVNCS1.0.1 & $1.01( \pm 0.13)$ & KVNCS1.0.1 & $1.07( \pm 0.26)$ & 1.320 \\
\hline $\mathrm{J} 0152+2207$ & $0149+218$ & $01: 52: 18.05$ & $22: 07: 07.68$ & KVNCS1.3.2 & $1.31( \pm 0.12)$ & KVNCS1.3.2 & $1.10( \pm 0.24)$ & 1.320 \\
\hline J0152+3350 & $0149+335$ & $01: 52: 34.57$ & $33: 50: 33.00$ & KVNCS1.3.1 & $0.16( \pm 0.02)$ & $\cdots$ & $\ldots$ & 2.431 \\
\hline $\mathrm{J} 0153+7115$ & $0149+710$ & $01: 53: 25.84$ & $71: 15: 06.48$ & KVNCS1.2.2 & $0.32( \pm 0.05)$ & $\cdots$ & $\cdots$ & 0.022 \\
\hline
\end{tabular}


Table 2 (continued)

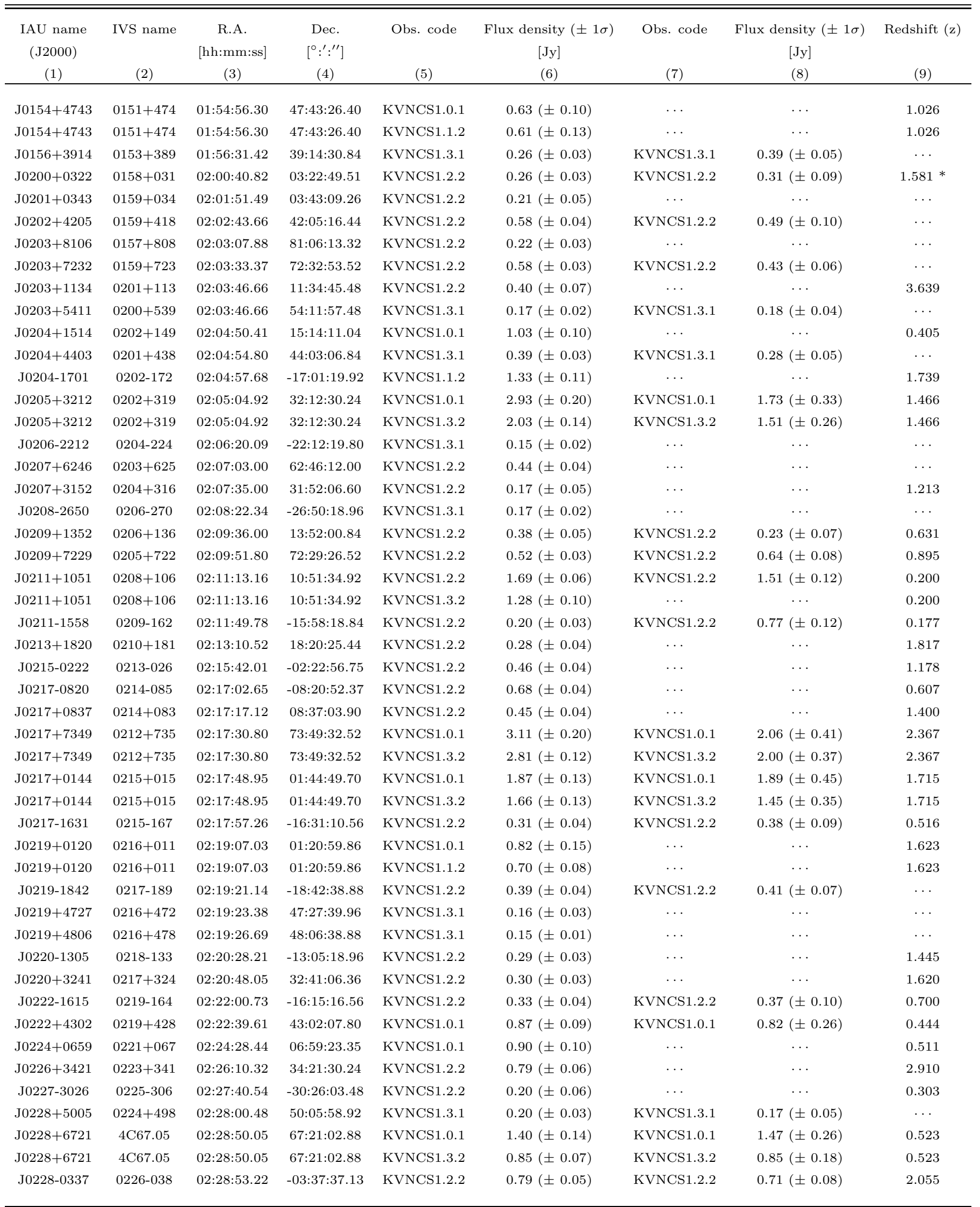


Table 2 (continued)

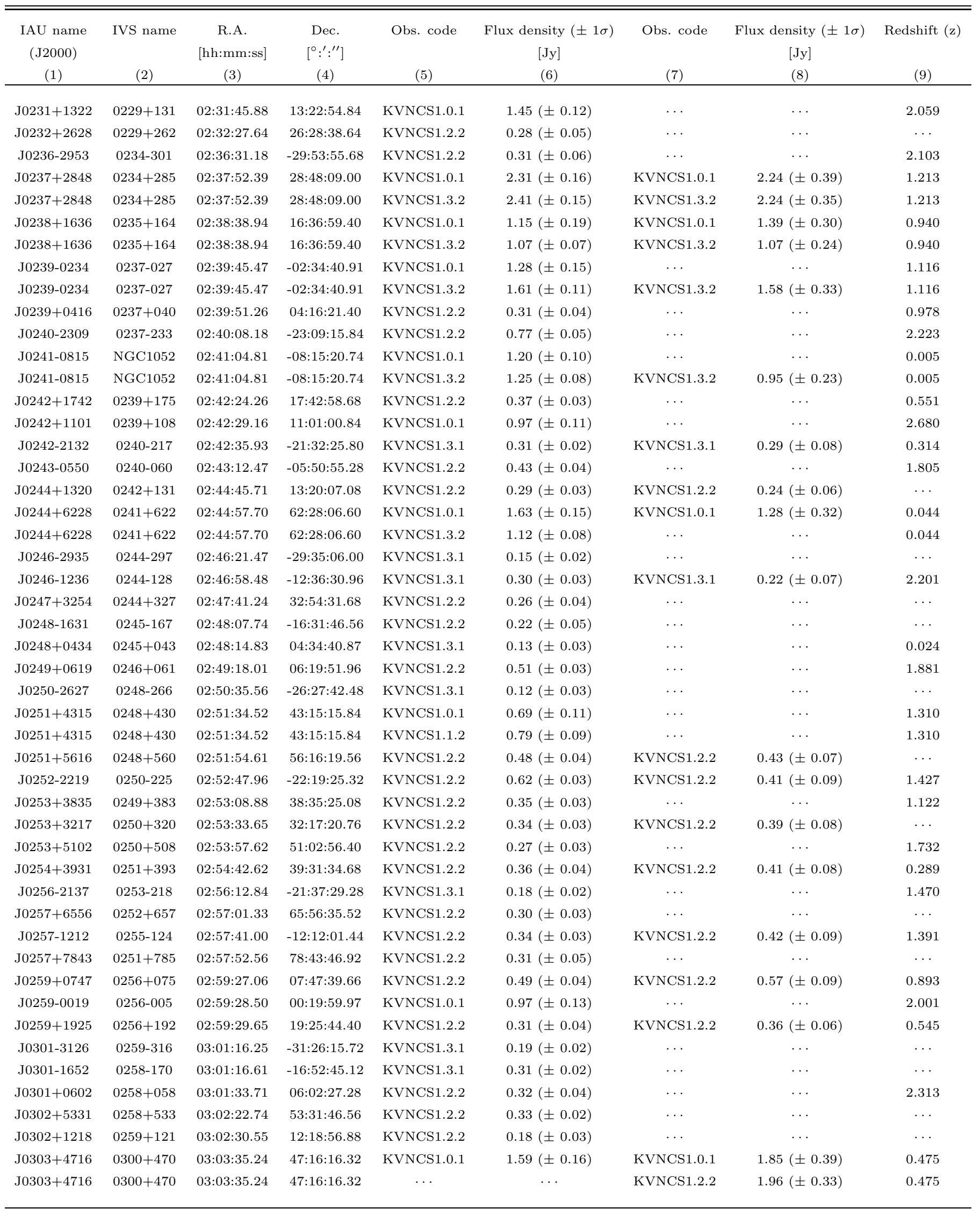


Table 2 (continued)

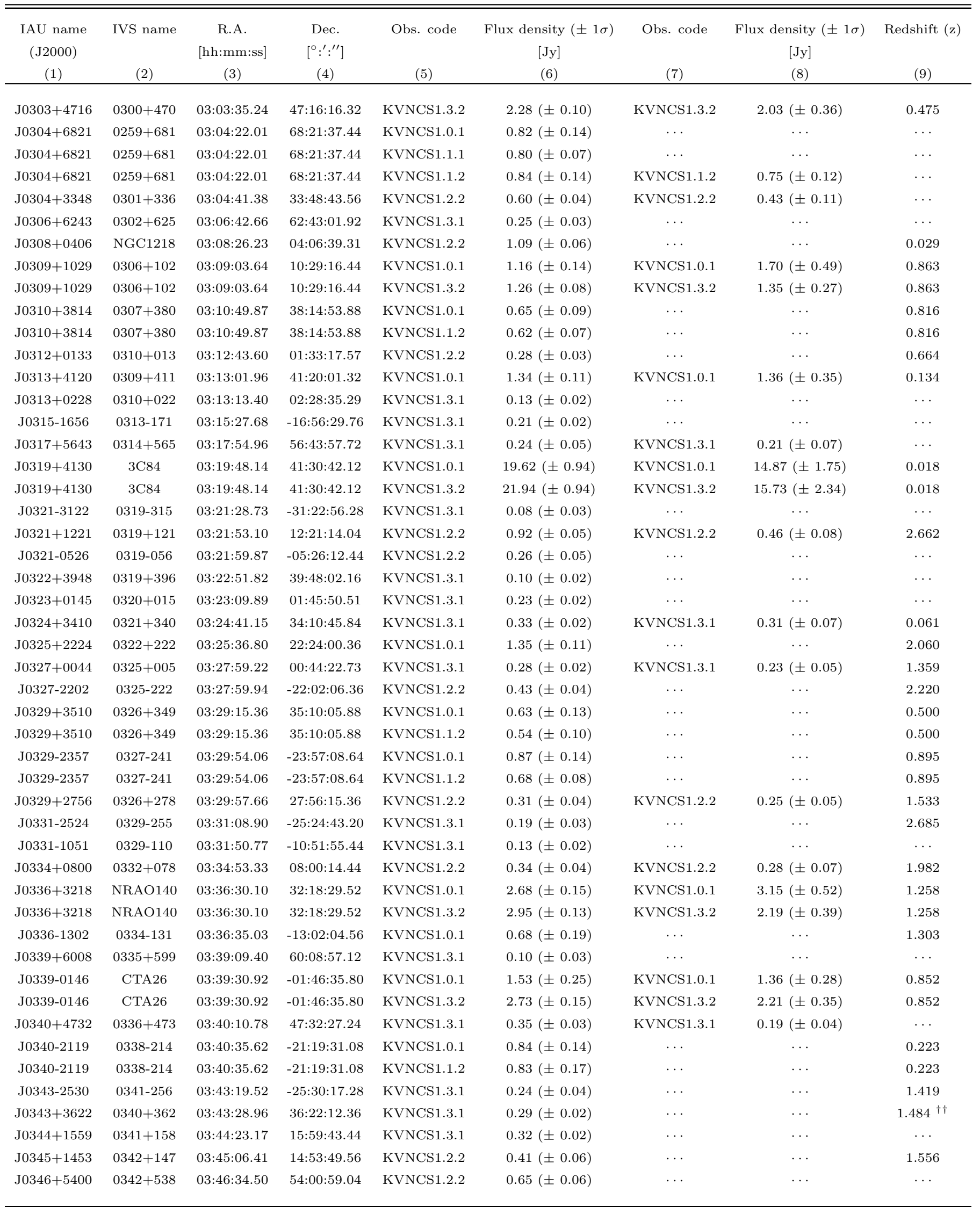


Table 2 (continued)

\begin{tabular}{|c|c|c|c|c|c|c|c|c|}
\hline J0347+5557 & $0344+558$ & $03: 47: 56.80$ & $55: 57: 31.68$ & KVNCS1.2.2 & $0.37( \pm 0.05)$ & $\ldots$ & $\cdots$ & $\cdots$ \\
\hline J0348-2749 & 0346-279 & $03: 48: 38.16$ & $-27: 49: 13.44$ & KVNCS1.0.1 & $0.84( \pm 0.14)$ & $\cdots$ & $\cdots$ & 0.991 \\
\hline J0348-2749 & 0346-279 & 03:48:38.16 & $-27: 49: 13.44$ & KVNCS1.1.2 & $0.94( \pm 0.15)$ & $\cdots$ & $\cdots$ & 0.991 \\
\hline J0349+4609 & $0345+460$ & $03: 49: 18.73$ & $46: 09: 59.76$ & KVNCS1.2.2 & $0.34( \pm 0.10)$ & KVNCS1.2.2 & $0.40( \pm 0.06)$ & 1.853 \\
\hline J0349-2102 & $0347-211$ & 03:49:57.83 & $-21: 02: 47.76$ & KVNCS1.2.2 & $0.42( \pm 0.06)$ & KVNCS1.2.2 & $0.28( \pm 0.06)$ & 2.944 \\
\hline J0351-1153 & 0348-120 & 03:51:10.98 & $-11: 53: 22.56$ & KVNCS1.2.2 & $0.31( \pm 0.04)$ & $\cdots$ & $\ldots$ & 1.520 \\
\hline J0352-2514 & 0350-253 & 03:52:11.06 & $-25: 14: 50.28$ & KVNCS1.0.1 & $0.73( \pm 0.14)$ & $\cdots$ & $\cdots$ & $\ldots$ \\
\hline J0354+6621 & $0349+662$ & 03:54:03.71 & $66: 21: 26.28$ & KVNCS1.2.2 & $0.20( \pm 0.03)$ & $\ldots$ & $\ldots$ & $\ldots$ \\
\hline J0357+2319 & $0354+231$ & 03:57:21.60 & 23:19:53.76 & KVNCS1.2.2 & $0.62( \pm 0.05)$ & $\cdots$ & $\cdots$ & $\cdots$ \\
\hline J0357-0751 & 0355-079 & 03:57:43.31 & $-07: 51: 14.58$ & KVNCS1.2.2 & $0.26( \pm 0.05)$ & KVNCS1.2.2 & $0.47( \pm 0.08)$ & 1.052 \\
\hline J0358+5606 & $0354+559$ & 03:58:30.18 & $56: 06: 44.64$ & KVNCS1.2.2 & $0.83( \pm 0.06)$ & KVNCS1.2.2 & $0.80( \pm 0.13)$ & $\ldots$ \\
\hline J0359+6005 & $0354+599$ & 03:59:02.65 & $60: 05: 22.20$ & KVNCS1.0.1 & $1.46( \pm 0.12)$ & KVNCS1.0.1 & $1.60( \pm 0.28)$ & $\cdots$ \\
\hline J0359+6005 & $0354+599$ & 03:59:02.65 & $60: 05: 22.20$ & KVNCS1.3.2 & $1.26( \pm 0.10)$ & KVNCS1.3.2 & $1.13( \pm 0.28)$ & $\cdots$ \\
\hline $\mathrm{J} 0359+2758$ & $0356+278$ & 03:59:27.92 & $27: 58: 23.88$ & KVNCS1.3.1 & $0.16( \pm 0.02)$ & KVNCS1.3.1 & $0.67( \pm 0.07)$ & 1.157 \\
\hline $\mathrm{J} 0359+5057$ & NRAO150 & 03:59:29.76 & $50: 57: 50.04$ & KVNCS1.0.1 & $15.19( \pm 0.64)$ & KVNCS1.0.1 & $12.73( \pm 1.49)$ & 1.520 \\
\hline $\mathrm{J} 0359+5057$ & NRAO150 & 03:59:29.76 & $50: 57: 50.04$ & KVNCS1.3.2 & $9.13( \pm 0.45)$ & KVNCS1.3.2 & $6.63( \pm 0.96)$ & 1.520 \\
\hline J0359+3220 & $0356+322$ & 03:59:44.92 & $32: 20: 47.04$ & KVNCS1.0.1 & $0.61( \pm 0.12)$ & $\ldots$ & $\ldots$ & 1.331 \\
\hline $\mathrm{J} 0359+3220$ & $0356+322$ & 03:59:44.92 & $32: 20: 47.04$ & KVNCS1.1.1 & $0.63( \pm 0.08)$ & $\ldots$ & $\cdots$ & 1.331 \\
\hline $\mathrm{J} 0359+3220$ & $0356+322$ & 03:59:44.92 & $32: 20: 47.04$ & KVNCS1.1.2 & $0.59( \pm 0.14)$ & KVNCS1.1.2 & $0.63( \pm 0.12)$ & 1.331 \\
\hline J0405-1308 & 0403-132 & 04:05:34.01 & $-13: 08: 13.56$ & KVNCS1.0.1 & $1.47( \pm 0.25)$ & KVNCS1.0.1 & $1.07( \pm 0.28)$ & 0.571 \\
\hline J0405-1308 & 0403-132 & 04:05:34.01 & $-13: 08: 13.56$ & KVNCS1.3.2 & $1.42( \pm 0.09)$ & $\ldots$ & $\ldots$ & 0.571 \\
\hline J0405+3803 & $0402+379$ & $04: 05: 49.27$ & $38: 03: 32.40$ & $\mathrm{KVNCS} 1.2 .2$ & $0.43( \pm 0.05)$ & $\cdots$ & $\cdots$ & 0.055 \\
\hline J0406+0637 & $0403+064$ & 04:06:34.31 & $06: 37: 14.99$ & KVNCS1.3.1 & $0.37( \pm 0.03)$ & KVNCS1.3.1 & $0.27( \pm 0.07)$ & 0.666 \\
\hline J0407+0742 & $0404+075$ & 04:07:29.10 & 07:42:07.49 & $\mathrm{KVNCS} 1.2 .2$ & $0.50( \pm 0.04)$ & KVNCS1.2.2 & $0.39( \pm 0.07)$ & 1.133 \\
\hline J0407-1211 & 0405-123 & 04:07:48.43 & $-12: 11: 36.60$ & KVNCS1.0.1 & $1.60( \pm 0.26)$ & KVNCS1.0.1 & $1.12( \pm 0.31)$ & 0.573 \\
\hline J0407-1211 & 0405-123 & 04:07:48.43 & $-12: 11: 36.60$ & KVNCS1.3.2 & $1.20( \pm 0.09)$ & KVNCS1.3.2 & $0.93( \pm 0.26)$ & 0.573 \\
\hline J0408-0529 & 0406-056 & 04:08:59.64 & $-05: 29: 40.52$ & KVNCS1.2.2 & $0.19( \pm 0.04)$ & $\ldots$ & $\ldots$ & 0.304 \\
\hline J0409-1238 & 0406-127 & 04:09:05.76 & $-12: 38: 48.12$ & KVNCS1.2.2 & $0.29( \pm 0.04)$ & $\cdots$ & $\cdots$ & 1.563 \\
\hline J0409+1217 & $0406+121$ & 04:09:22.00 & $12: 17: 39.84$ & $\mathrm{KVNCS} 1.2 .2$ & $0.33( \pm 0.03)$ & $\cdots$ & $\cdots$ & 0.504 \\
\hline J0410+7656 & $0403+768$ & $04: 10: 45.62$ & $76: 56: 45.24$ & KVNCS1.3.1 & $1.17( \pm 0.05)$ & KVNCS1.3.1 & $0.92( \pm 0.06)$ & 0.599 \\
\hline J0411-2758 & $0409-280$ & $04: 11: 25.12$ & $-27: 58: 16.68$ & KVNCS1.3.1 & $0.15( \pm 0.03)$ & $\ldots$ & $\ldots$ & $\ldots$ \\
\hline $\mathrm{J} 0412+0010$ & $0409+000$ & 04:12:33.44 & 00:10:48.49 & KVNCS1.3.1 & $0.22( \pm 0.02)$ & KVNCS1.3.1 & $0.20( \pm 0.06)$ & 1.130 \\
\hline J0412+2305 & $0409+229$ & $04: 12: 43.67$ & $23: 05: 05.28$ & KVNCS1.2.2 & $0.35( \pm 0.05)$ & KVNCS1.2.2 & $0.30( \pm 0.05)$ & 1.215 \\
\hline J0413+5250 & $0409+527$ & 04:13:37.09 & $52: 50: 52.80$ & KVNCS1.3.2 & $0.24( \pm 0.02)$ & $\ldots$ & $\ldots$ & $\cdots$ \\
\hline J0414+3418 & $0411+341$ & $04: 14: 37.25$ & $34: 18: 51.12$ & $\mathrm{KVNCS} 1.2 .2$ & $0.84( \pm 0.11)$ & KVNCS1.2.2 & $0.39( \pm 0.10)$ & $\cdots$ \\
\hline J0415+4452 & $0412+447$ & $04: 15: 56.52$ & $44: 52: 49.80$ & KVNCS1.1.1 & $0.37( \pm 0.09)$ & $\cdots$ & $\ldots$ & 0.485 \\
\hline J0415+4452 & $0412+447$ & $04: 15: 56.52$ & $44: 52: 49.80$ & KVNCS1.1.2 & $0.48( \pm 0.08)$ & $\cdots$ & $\cdots$ & 0.485 \\
\hline
\end{tabular}


Table 2 (continued)

\begin{tabular}{|c|c|c|c|c|c|c|c|c|}
\hline $\begin{array}{l}\text { IAU name } \\
(\mathrm{J} 2000) \\
(1) \\
\end{array}$ & IVS name & $\begin{array}{c}\text { R.A. } \\
\text { [hh:mm:ss] } \\
(3)\end{array}$ & $\begin{array}{c}\text { Dec. } \\
{\left[{ }^{\circ}:^{\prime}:^{\prime \prime}\right]} \\
(4) \\
\end{array}$ & Obs. code & $\begin{array}{c}\text { Flux density }( \pm 1 \sigma) \\
{[\mathrm{Jy}]} \\
(6) \\
\end{array}$ & Obs. code & $\begin{array}{c}\text { Flux density }( \pm 1 \sigma) \\
{\left[\begin{array}{c}\mathrm{Jy}] \\
(8)\end{array}\right.} \\
\end{array}$ & Redshift (z) \\
\hline J0416-1851 & 0414-189 & $04: 16: 36.55$ & $-18: 51: 08.28$ & KVNCS1.0.1 & $0.81( \pm 0.16)$ & $\cdots$ & $\cdots$ & 1.536 \\
\hline J0416-1851 & 0414-189 & $04: 16: 36.55$ & $-18: 51: 08.28$ & KVNCS1.1.2 & $0.83( \pm 0.09)$ & $\cdots$ & $\cdots$ & 1.536 \\
\hline J0419+3955 & $0415+398$ & 04:19:22.55 & $39: 55: 28.92$ & KVNCS1.3.1 & $0.35( \pm 0.02)$ & KVNCS1.3.1 & $0.31( \pm 0.10)$ & $\ldots$ \\
\hline J0422+5324 & $0418+532$ & $04: 22: 44.40$ & $53: 24: 26.28$ & KVNCS1.3.2 & $0.41( \pm 0.04)$ & KVNCS1.3.2 & $4.48( \pm 0.81)$ & 1.273 \\
\hline J0422+0219 & $0420+022$ & $04: 22: 52.21$ & 02:19:26.94 & KVNCS1.0.1 & $0.84( \pm 0.12)$ & $\ldots$ & $\ldots$ & 2.277 \\
\hline J0423-0120 & 0420-014 & $04: 23: 15.79$ & $-01: 20: 33.07$ & KVNCS1.0.1 & $8.90( \pm 1.14)$ & KVNCS1.0.1 & $7.93( \pm 0.92)$ & 0.916 \\
\hline J0423-0120 & 0420-014 & $04: 23: 15.79$ & $-01: 20: 33.07$ & KVNCS1.3.2 & $5.17( \pm 0.20)$ & KVNCS1.3.2 & $4.28( \pm 0.74)$ & 0.916 \\
\hline J0423+4150 & $0420+417$ & 04:23:56.00 & $41: 50: 02.76$ & KVNCS1.0.1 & $1.04( \pm 0.10)$ & $\ldots$ & $\ldots$ & $\cdots$ \\
\hline J0424+0805 & $0422+079$ & $04: 24: 57.60$ & 08:05:17.34 & KVNCS1.3.1 & $0.16( \pm 0.02)$ & $\cdots$ & $\cdots$ & 3.085 \\
\hline J0426+6825 & $0421+683$ & 04:26:50.06 & $68: 25: 53.04$ & KVNCS1.3.1 & $0.33( \pm 0.02)$ & $\ldots$ & $\ldots$ & $\ldots$ \\
\hline J0427-0700 & $0425-071$ & $04: 27: 26.17$ & $-07: 00: 31.25$ & KVNCS1.3.1 & $0.15( \pm 0.02)$ & $\cdots$ & $\cdots$ & $\ldots$ \\
\hline J0427+4133 & $0424+414$ & 04:27:46.04 & 41:33:01.08 & KVNCS1.0.1 & $0.53( \pm 0.09)$ & $\cdots$ & $\cdots$ & $\ldots$ \\
\hline J0427+4133 & $0424+414$ & $04: 27: 46.04$ & 41:33:01.08 & KVNCS1.1.1 & $0.49( \pm 0.07)$ & $\cdots$ & $\cdots$ & $\cdots$ \\
\hline J0427+4133 & $0424+414$ & $04: 27: 46.04$ & 41:33:01.08 & KVNCS1.1.2 & $0.63( \pm 0.08)$ & $\cdots$ & $\cdots$ & $\cdots$ \\
\hline J0427+0457 & $0425+048$ & $04: 27: 47.56$ & 04:57:08.32 & KVNCS1.2.2 & $0.34( \pm 0.04)$ & KVNCS1.2.2 & $0.51( \pm 0.10)$ & 0.517 \\
\hline J0428+3259 & $0424+328$ & 04:28:05.81 & $32: 59: 52.08$ & KVNCS1.1.1 & $0.27( \pm 0.07)$ & $\ldots$ & $\ldots$ & 0.476 \\
\hline J0429+2724 & $0426+273$ & $04: 29: 52.94$ & $27: 24: 37.80$ & KVNCS1.2.2 & $0.55( \pm 0.05)$ & KVNCS1.2.2 & $0.26( \pm 0.08)$ & $\ldots$ \\
\hline J0431-0406 & $0428-042$ & 04:31:28.09 & $-04: 06: 27.32$ & KVNCS1.3.1 & $0.15( \pm 0.02)$ & $\ldots$ & $\ldots$ & $\cdots$ \\
\hline J0432-1614 & 0430-163 & 04:32:29.08 & $-16: 14: 05.64$ & KVNCS1.2.2 & $0.22( \pm 0.05)$ & $\cdots$ & $\ldots$ & $\cdots$ \\
\hline J0437-1844 & 0434-188 & 04:37:01.49 & $-18: 44: 48.48$ & KVNCS1.1.2 & $0.40( \pm 0.09)$ & $\cdots$ & $\cdots$ & 2.702 \\
\hline J0437-2954 & 0435-300 & $04: 37: 36.55$ & $-29: 54: 03.96$ & KVNCS1.2.2 & $0.34( \pm 0.04)$ & $\ldots$ & $\cdots$ & 1.328 \\
\hline J0438+3004 & $0434+299$ & 04:38:04.96 & $30: 04: 45.48$ & KVNCS1.2.2 & $0.35( \pm 0.04)$ & $\cdots$ & $\cdots$ & 1.454 \\
\hline J0438-1251 & 0436-129 & 04:38:35.02 & $-12: 51: 03.24$ & KVNCS1.2.2 & $0.45( \pm 0.06)$ & KVNCS1.2.2 & $0.41( \pm 0.10)$ & 1.277 \\
\hline J0438-0848 & 0436-089 & $04: 38: 37.86$ & $-08: 48: 21.49$ & KVNCS1.3.1 & $0.14( \pm 0.03)$ & $\cdots$ & $\ldots$ & $\cdots$ \\
\hline J0438+2153 & $0435+217$ & 04:38:55.90 & $21: 53: 10.32$ & KVNCS1.2.2 & $0.23( \pm 0.05)$ & KVNCS1.2.2 & $0.36( \pm 0.08)$ & $\cdots$ \\
\hline J0439+0520 & $0436+052$ & $04: 39: 02.27$ & $05: 20: 43.69$ & KVNCS1.3.1 & $0.30( \pm 0.02)$ & $\ldots$ & $\ldots$ & 0.208 \\
\hline J0440+4244 & $0436+426$ & 04:40:07.86 & $42: 44: 40.20$ & KVNCS1.3.1 & $0.16( \pm 0.03)$ & $\ldots$ & $\ldots$ & $\ldots$ \\
\hline J0440+1437 & $0437+145$ & 04:40:21.14 & $14: 37: 57.00$ & KVNCS1.2.2 & $0.42( \pm 0.07)$ & KVNCS1.2.2 & $0.41( \pm 0.07)$ & $\cdots$ \\
\hline J0440+2728 & $0437+273$ & $04: 40: 27.73$ & $27: 28: 40.80$ & KVNCS1.3.1 & $0.23( \pm 0.02)$ & $\ldots$ & $\ldots$ & $\cdots$ \\
\hline J0442-2825 & $0440-285$ & $04: 42: 37.66$ & $-28: 25: 30.72$ & KVNCS1.2.2 & $0.45( \pm 0.07)$ & $\cdots$ & $\ldots$ & 1.952 \\
\hline J0442-0017 & NRAO190 & $04: 42: 38.66$ & $00: 17: 43.42$ & KVNCS1.0.1 & $1.28( \pm 0.21)$ & $\ldots$ & $\cdots$ & 0.844 \\
\hline J0446+3900 & $0442+389$ & $04: 46: 11.50$ & $39: 00: 16.92$ & KVNCS1.1.1 & $0.30( \pm 0.05)$ & $\cdots$ & $\cdots$ & 0.413 \\
\hline J0448+0950 & $0445+097$ & $04: 48: 21.74$ & 09:50:51.47 & $\ldots$ & $\ldots$ & KVNCS1.2.2 & $0.41( \pm 0.07)$ & 2.108 \\
\hline J0448+0950 & $0445+097$ & $04: 48: 21.74$ & 09:50:51.47 & KVNCS1.3.1 & $0.43( \pm 0.03)$ & KVNCS1.3.1 & $0.34( \pm 0.06)$ & 2.108 \\
\hline J0448+3629 & $0445+364$ & $04: 48: 35.17$ & $36: 29: 31.56$ & KVNCS1.3.1 & $0.16( \pm 0.02)$ & KVNCS1.3.1 & $0.16( \pm 0.05)$ & $\cdots$ \\
\hline J0448+1127 & $0446+113$ & $04: 48: 50.40$ & $11: 27: 54.36$ & KVNCS1.3.1 & $0.36( \pm 0.02)$ & KVNCS1.3.1 & $0.37( \pm 0.06)$ & 1.375 \\
\hline J0449+1121 & $0446+112$ & 04:49:07.68 & $11: 21: 28.44$ & KVNCS1.0.1 & $1.38( \pm 0.14)$ & KVNCS1.0.1 & $1.21( \pm 0.34)$ & 1.207 \\
\hline
\end{tabular}


Table 2 (continued)

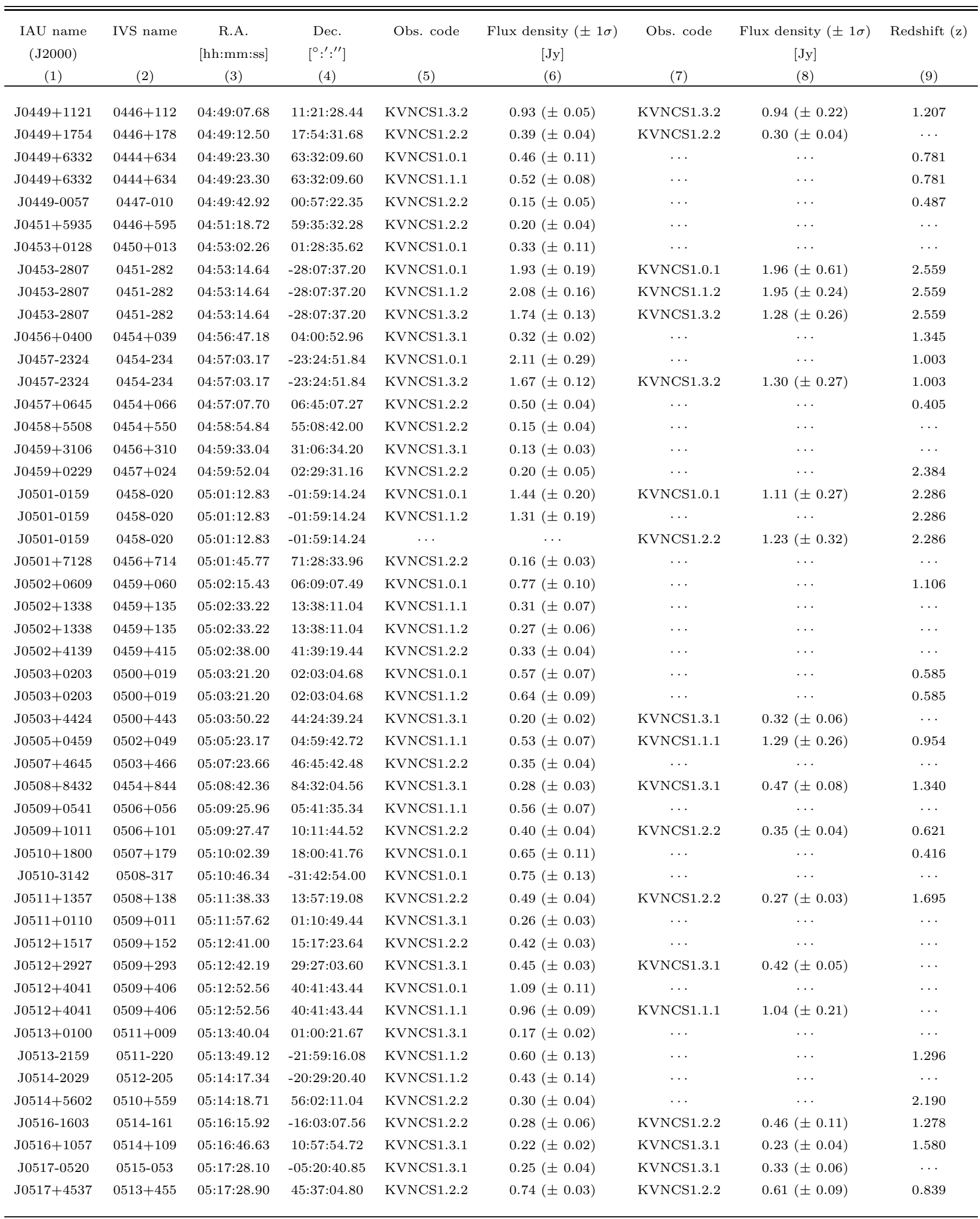


Table 2 (continued)

\begin{tabular}{|c|c|c|c|c|c|c|c|c|}
\hline $\begin{array}{l}\text { IAU name } \\
(\mathrm{J} 2000) \\
(1) \\
\end{array}$ & IVS name & $\begin{array}{c}\text { R.A. } \\
\text { [hh:mm:ss] } \\
(3)\end{array}$ & $\begin{array}{c}\text { Dec. } \\
{\left[{ }^{\circ}:^{\prime}:^{\prime \prime}\right]} \\
(4) \\
\end{array}$ & Obs. code & $\begin{array}{c}\text { Flux density }( \pm 1 \sigma) \\
{[\mathrm{Jy}]} \\
(6) \\
\end{array}$ & Obs. code & $\begin{array}{c}\text { Flux density }( \pm 1 \sigma) \\
{[\mathrm{Jy}]} \\
(8) \\
\end{array}$ & Redshift (z) \\
\hline J0517+0648 & $0515+067$ & $05: 17: 51.36$ & 06:48:03.20 & KVNCS1.3.1 & $0.22( \pm 0.02)$ & $\cdots$ & $\cdots$ & 0.840 \\
\hline J0518+2054 & $0515+208$ & 05:18:03.82 & $20: 54: 52.56$ & KVNCS1.2.2 & $0.27( \pm 0.05)$ & $\cdots$ & $\cdots$ & 2.579 \\
\hline J0518+3306 & $0514+330$ & 05:18:05.15 & 33:06:13.32 & KVNCS1.2.2 & $0.32( \pm 0.04)$ & KVNCS1.2.2 & $0.33( \pm 0.06)$ & $\cdots$ \\
\hline $\mathrm{J} 0521+2112$ & $0518+211$ & $05: 21: 45.97$ & $21: 12: 51.48$ & KVNCS1.2.2 & $0.39( \pm 0.03)$ & KVNCS1.2.2 & $0.41( \pm 0.06)$ & $\ldots$ \\
\hline J0522+0113 & $0519+011$ & $05: 22: 17.47$ & 01:13:31.19 & KVNCS1.2.2 & $0.28( \pm 0.03)$ & KVNCS1.2.2 & $0.46( \pm 0.07)$ & 2.941 \\
\hline J0523-2614 & $0521-262$ & $05: 23: 18.46$ & $-26: 14: 09.60$ & KVNCS1.2.2 & $0.15( \pm 0.05)$ & $\ldots$ & $\ldots$ & $\ldots$ \\
\hline J0525-2338 & $0523-236$ & $05: 25: 06.49$ & $-23: 38: 10.68$ & KVNCS1.0.1 & $1.15( \pm 0.20)$ & $\cdots$ & $\cdots$ & 3.100 \\
\hline J0527+0331 & $0524+034$ & $05: 27: 32.69$ & 03:31:31.51 & KVNCS1.0.1 & $0.63( \pm 0.11)$ & $\cdots$ & $\cdots$ & $\cdots$ \\
\hline J0530+1331 & $0528+134$ & 05:30:56.41 & $13: 31: 55.20$ & KVNCS1.0.1 & $1.56( \pm 0.14)$ & KVNCS1.0.1 & $1.37( \pm 0.36)$ & 2.060 \\
\hline J0530+1331 & $0528+134$ & $05: 30: 56.41$ & $13: 31: 55.20$ & KVNCS1.3.2 & $1.22( \pm 0.05)$ & KVNCS1.3.2 & $1.29( \pm 0.28)$ & 2.060 \\
\hline J0532-0307 & 0529-031 & $05: 32: 07.51$ & $-03: 07: 07.03$ & KVNCS1.3.1 & $0.25( \pm 0.03)$ & $\ldots$ & $\ldots$ & $\ldots$ \\
\hline J0532+0732 & $0529+075$ & 05:32:39.01 & $07: 32: 43.33$ & KVNCS1.0.1 & $1.62( \pm 0.18)$ & $\cdots$ & $\cdots$ & 1.254 \\
\hline J0532+0732 & $0529+075$ & 05:32:39.01 & $07: 32: 43.33$ & KVNCS1.1.2 & $1.22( \pm 0.13)$ & $\ldots$ & $\ldots$ & 1.254 \\
\hline J0532+0732 & $0529+075$ & 05:32:39.01 & $07: 32: 43.33$ & $\ldots$ & $\ldots$ & KVNCS1.2.2 & $1.28( \pm 0.33)$ & 1.254 \\
\hline J0532+0732 & $0529+075$ & 05:32:39.01 & $07: 32: 43.33$ & KVNCS1.3.2 & $1.31( \pm 0.09)$ & KVNCS1.3.2 & $1.12( \pm 0.28)$ & 1.254 \\
\hline J0533+4822 & $0529+483$ & $05: 33: 15.88$ & $48: 22: 52.68$ & KVNCS1.0.1 & $1.55( \pm 0.11)$ & KVNCS1.0.1 & $1.59( \pm 0.40)$ & 1.162 \\
\hline J0533+4822 & $0529+483$ & $05: 33: 15.88$ & $48: 22: 52.68$ & KVNCS1.3.2 & $1.75( \pm 0.08)$ & KVNCS1.3.2 & $1.46( \pm 0.30)$ & 1.162 \\
\hline J0536+5038 & $0532+506$ & $05: 36: 20.23$ & $50: 38: 26.16$ & KVNCS1.0.1 & $0.42( \pm 0.11)$ & $\ldots$ & $\ldots$ & $\ldots$ \\
\hline J0536+5038 & $0532+506$ & $05: 36: 20.23$ & $50: 38: 26.16$ & KVNCS1.1.1 & $0.35( \pm 0.07)$ & $\cdots$ & $\cdots$ & $\cdots$ \\
\hline $\mathrm{J} 0541+5312$ & $0537+531$ & 05:41:16.19 & $53: 12: 24.84$ & KVNCS1.0.1 & $0.44( \pm 0.09)$ & $\cdots$ & $\cdots$ & 1.275 \\
\hline $\mathrm{J} 0541+5312$ & $0537+531$ & 05:41:16.19 & $53: 12: 24.84$ & KVNCS1.1.1 & $0.45( \pm 0.04)$ & $\cdots$ & $\cdots$ & 1.275 \\
\hline J0541-0541 & 0539-057 & 05:41:38.08 & $-05: 41: 49.42$ & KVNCS1.0.1 & $0.97( \pm 0.19)$ & $\cdots$ & $\cdots$ & 0.839 \\
\hline J0541-0541 & 0539-057 & 05:41:38.08 & $-05: 41: 49.42$ & KVNCS1.1.2 & $0.87( \pm 0.13)$ & $\cdots$ & $\ldots$ & 0.839 \\
\hline J0542+4951 & $3 \mathrm{C} 147$ & $05: 42: 36.14$ & 49:51:07.20 & KVNCS1.0.1 & $2.04( \pm 0.15)$ & KVNCS1.0.1 & $1.30( \pm 0.30)$ & 0.545 \\
\hline J0542+4951 & $3 \mathrm{C} 147$ & $05: 42: 36.14$ & $49: 51: 07.20$ & KVNCS1.3.2 & $1.91( \pm 0.07)$ & $\ldots$ & $\ldots$ & 0.545 \\
\hline J0542-0913 & 0540-092 & $05: 42: 55.87$ & $-09: 13: 31.01$ & KVNCS1.2.2 & $0.96( \pm 0.06)$ & KVNCS1.2.2 & $0.73( \pm 0.11)$ & $\cdots$ \\
\hline $\mathrm{J} 0547+2721$ & $0544+273$ & $05: 47: 34.15$ & $27: 21: 56.88$ & KVNCS1.0.1 & $0.67( \pm 0.12)$ & $\ldots$ & $\ldots$ & $\cdots$ \\
\hline $\mathrm{J} 0547+2721$ & $0544+273$ & $05: 47: 34.15$ & $27: 21: 56.88$ & KVNCS1.1.1 & $0.61( \pm 0.08)$ & $\cdots$ & $\cdots$ & $\cdots$ \\
\hline J0550+2326 & $0547+234$ & 05:50:47.40 & $23: 26: 48.12$ & KVNCS1.2.2 & $0.36( \pm 0.04)$ & $\cdots$ & $\cdots$ & $\cdots$ \\
\hline J0551+0829 & $0548+084$ & $05: 51: 11.23$ & 08:29:11.22 & KVNCS1.2.2 & $0.26( \pm 0.05)$ & $\cdots$ & $\ldots$ & $\cdots$ \\
\hline J0552+3754 & $0548+378$ & $05: 52: 17.94$ & $37: 54: 25.20$ & KVNCS1.2.2 & $0.42( \pm 0.07)$ & KVNCS1.2.2 & $0.32( \pm 0.07)$ & 0.815 \\
\hline J0552+0313 & $0550+032$ & $05: 52: 50.09$ & 03:13:27.23 & KVNCS1.0.1 & $0.48( \pm 0.08)$ & $\cdots$ & $\cdots$ & $\cdots$ \\
\hline J0554+6857 & $0548+689$ & 05:54:00.79 & $68: 57: 54.36$ & KVNCS1.2.2 & $0.26( \pm 0.05)$ & $\ldots$ & $\cdots$ & 1.372 \\
\hline J0555+3948 & $0552+398$ & $05: 55: 30.79$ & $39: 48: 49.32$ & KVNCS1.0.1 & $3.57( \pm 0.20)$ & KVNCS1.0.1 & $2.33( \pm 0.55)$ & 2.365 \\
\hline J0555+3948 & $0552+398$ & $05: 55: 30.79$ & $39: 48: 49.32$ & KVNCS1.3.2 & $3.08( \pm 0.11)$ & KVNCS1.3.2 & $2.04( \pm 0.39)$ & 2.365 \\
\hline $\mathrm{J} 0557+2413$ & $0554+242$ & $05: 57: 04.72$ & $24: 13: 55.20$ & KVNCS1.0.1 & $0.44( \pm 0.14)$ & $\cdots$ & $\cdots$ & $\cdots$ \\
\hline $\mathrm{J} 0557+2413$ & $0554+242$ & $05: 57: 04.72$ & $24: 13: 55.20$ & KVNCS1.1.1 & $0.51( \pm 0.08)$ & KVNCS1.1.1 & $0.82( \pm 0.20)$ & $\cdots$ \\
\hline
\end{tabular}


Table 2 (continued)

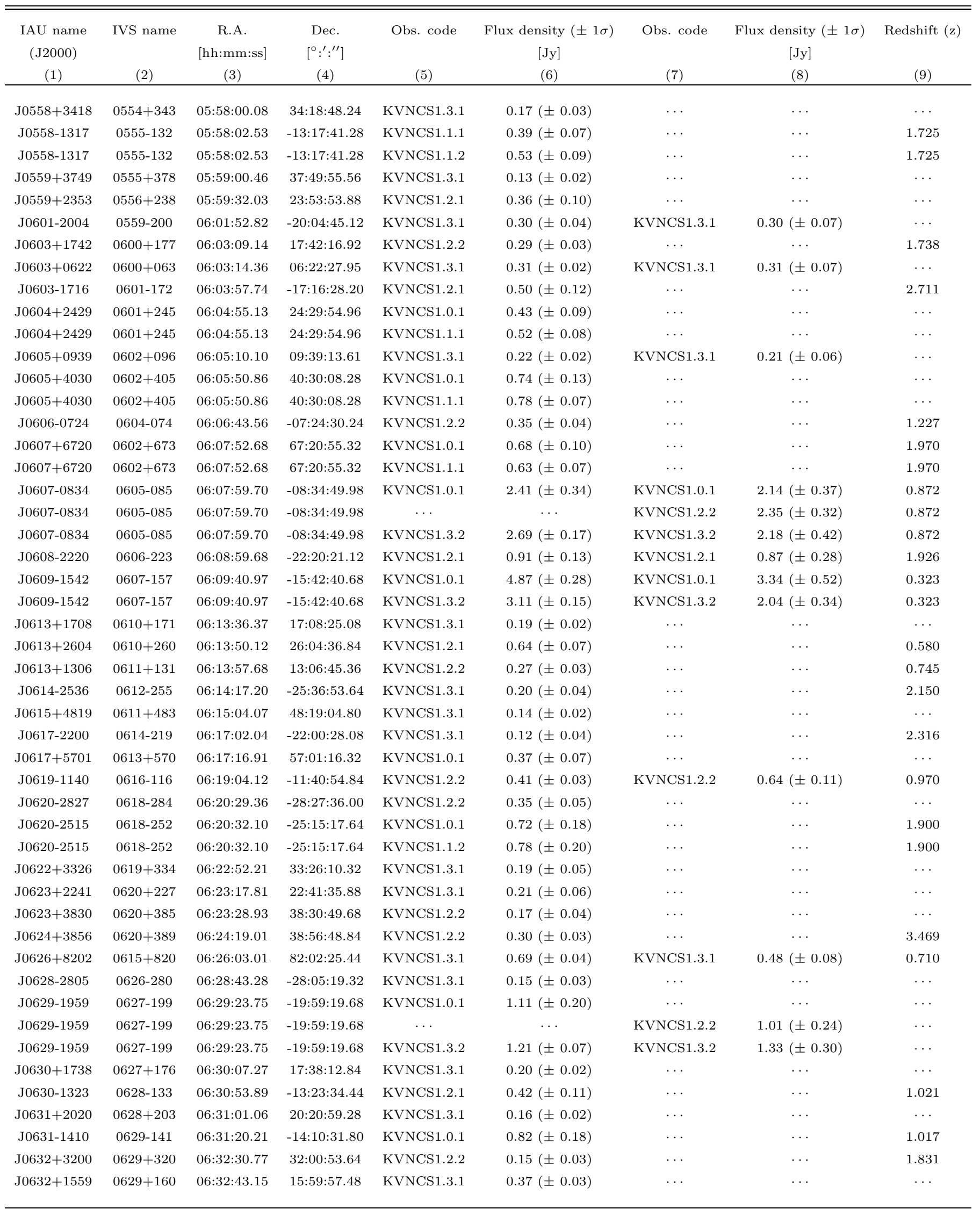


Table 2 (continued)

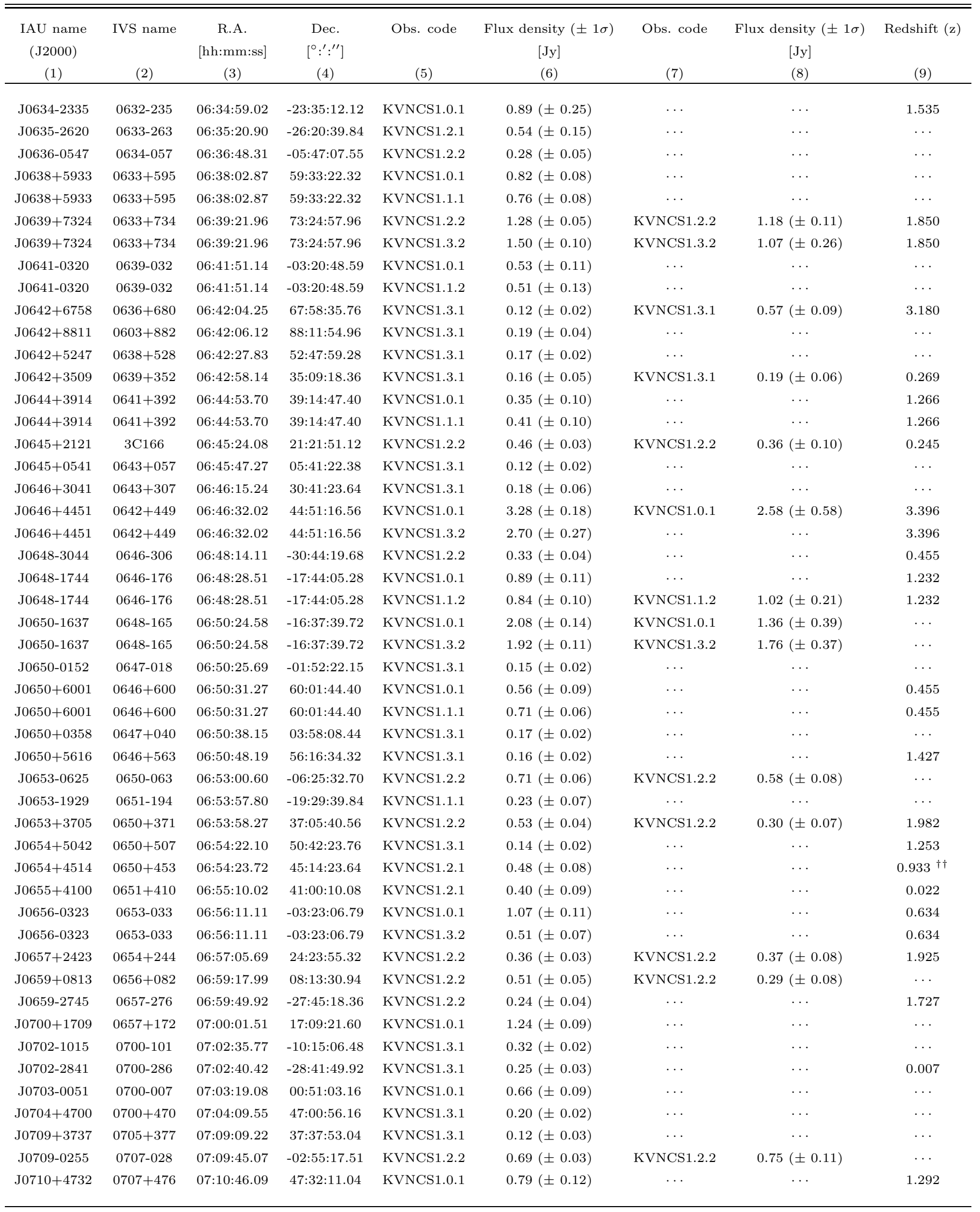


Table 2 (continued)

\begin{tabular}{|c|c|c|c|c|c|c|c|c|}
\hline $\begin{array}{l}\text { IAU name } \\
(\mathrm{J} 2000) \\
(1) \\
\end{array}$ & IVS name & $\begin{array}{c}\text { R.A. } \\
\text { [hh:mm:ss] } \\
(3)\end{array}$ & $\begin{array}{c}\text { Dec. } \\
{\left[{ }^{\circ}:^{\prime}:^{\prime \prime}\right]} \\
(4) \\
\end{array}$ & Obs. code & $\begin{array}{c}\text { Flux density }( \pm 1 \sigma) \\
{[\mathrm{Jy}]} \\
(6) \\
\end{array}$ & Obs. code & $\begin{array}{c}\text { Flux density }( \pm 1 \sigma) \\
{[\mathrm{Jy}]} \\
(8) \\
\end{array}$ & Redshift (z) \\
\hline J0712+5033 & $0708+506$ & $07: 12: 43.67$ & $50: 33: 22.68$ & KVNCS1.2.2 & $0.40( \pm 0.06)$ & KVNCS1.1.2 & $0.77( \pm 0.25)$ & $\cdots$ \\
\hline J0713+4349 & $0710+439$ & $07: 13: 38.17$ & 43:49:17.04 & KVNCS1.2.1 & $0.63( \pm 0.08)$ & $\cdots$ & $\ldots$ & 0.518 \\
\hline J0713+1935 & $0710+196$ & $07: 13: 55.67$ & 19:35:00.24 & KVNCS1.0.1 & $0.36( \pm 0.11)$ & KVNCS1.1.2 & $3.34( \pm 0.45)$ & 0.540 \\
\hline J0717+4538 & $0714+457$ & $07: 17: 51.86$ & $45: 38: 03.12$ & KVNCS1.0.1 & $0.88( \pm 0.11)$ & $\cdots$ & $\cdots$ & 0.940 \\
\hline J0717+4538 & $0714+457$ & $07: 17: 51.86$ & $45: 38: 03.12$ & KVNCS1.1.1 & $0.72( \pm 0.08)$ & $\cdots$ & $\cdots$ & 0.940 \\
\hline J0718-1813 & $0716-181$ & $07: 18: 14.15$ & $-18: 13: 04.08$ & KVNCS1.3.1 & $0.17( \pm 0.03)$ & $\cdots$ & $\cdots$ & $\cdots$ \\
\hline J0719+3307 & $0716+332$ & $07: 19: 19.42$ & $33: 07: 09.84$ & KVNCS1.2.1 & $0.68( \pm 0.09)$ & $\cdots$ & $\cdots$ & 0.779 \\
\hline J0721+0406 & $0718+042$ & $07: 21: 23.90$ & $04: 06: 44.21$ & KVNCS1.2.2 & $0.54( \pm 0.06)$ & KVNCS1.2.2 & $0.62( \pm 0.12)$ & 0.665 \\
\hline J0725+1425 & $0722+145$ & $07: 25: 16.82$ & $14: 25: 13.80$ & KVNCS1.0.1 & $0.72( \pm 0.09)$ & $\ldots$ & $\ldots$ & 1.038 \\
\hline J0725-1904 & 0723-189 & $07: 25: 50.16$ & $-19: 04: 19.20$ & KVNCS1.2.2 & $0.38( \pm 0.04)$ & $\cdots$ & $\cdots$ & $\ldots$ \\
\hline J0725-0054 & 0723-008 & $07: 25: 50.63$ & $00: 54: 56.55$ & KVNCS1.0.1 & $3.07( \pm 0.17)$ & KVNCS1.0.1 & $3.09( \pm 0.64)$ & 0.128 \\
\hline J0725-0054 & 0723-008 & $07: 25: 50.63$ & $00: 54: 56.55$ & KVNCS1.3.2 & $1.44( \pm 0.09)$ & KVNCS1.3.2 & $1.62( \pm 0.30)$ & 0.128 \\
\hline J0726+7911 & $0718+793$ & $07: 26: 11.72$ & $79: 11: 30.84$ & KVNCS1.0.1 & $0.45( \pm 0.12)$ & $\ldots$ & $\ldots$ & $\cdots$ \\
\hline J0726+7911 & $0718+793$ & $07: 26: 11.72$ & $79: 11: 30.84$ & KVNCS1.1.2 & $0.50( \pm 0.12)$ & $\cdots$ & $\cdots$ & $\cdots$ \\
\hline J0726+2153 & $0723+219$ & $07: 26: 14.24$ & 21:53:20.04 & KVNCS1.2.2 & $0.36( \pm 0.02)$ & $\cdots$ & $\cdots$ & 1.857 \\
\hline J0728+6748 & $0723+679$ & $07: 28: 10.88$ & $67: 48: 47.16$ & KVNCS1.0.1 & $0.51( \pm 0.08)$ & $\cdots$ & $\cdots$ & 0.846 \\
\hline J0728+6748 & $0723+679$ & $07: 28: 10.88$ & $67: 48: 47.16$ & KVNCS1.1.1 & $0.48( \pm 0.09)$ & $\cdots$ & $\cdots$ & 0.846 \\
\hline J0728+5701 & $0724+571$ & $07: 28: 49.62$ & $57: 01: 24.24$ & KVNCS1.2.1 & $0.36( \pm 0.07)$ & $\cdots$ & $\cdots$ & 0.426 \\
\hline J0729-1320 & $0726-132$ & $07: 29: 17.81$ & $-13: 20: 02.40$ & KVNCS1.3.1 & $0.13( \pm 0.02)$ & $\ldots$ & $\ldots$ & $\cdots$ \\
\hline J0733+0456 & $0731+050$ & 07:33:57.46 & $04: 56: 14.50$ & KVNCS1.2.1 & $0.37( \pm 0.11)$ & $\cdots$ & $\cdots$ & 3.010 \\
\hline J0735+4750 & $0731+479$ & $07: 35: 02.33$ & $47: 50: 08.52$ & KVNCS1.2.1 & $0.38( \pm 0.07)$ & $\cdots$ & $\cdots$ & 0.782 \\
\hline J0735-1735 & 0733-174 & $07: 35: 45.82$ & $-17: 35: 48.48$ & KVNCS1.2.2 & $0.44( \pm 0.06)$ & $\cdots$ & $\cdots$ & $\cdots$ \\
\hline J0736+2954 & $0733+300$ & $07: 36: 13.64$ & $29: 54: 22.32$ & KVNCS1.3.1 & $0.21( \pm 0.02)$ & $\cdots$ & $\cdots$ & $\cdots$ \\
\hline J0736+2954 & $0733+300$ & $07: 36: 13.64$ & $29: 54: 22.32$ & KVNCS1.3.2 & $0.23( \pm 0.03)$ & $\cdots$ & $\cdots$ & $\ldots$ \\
\hline J0737-0720 & 0734-072 & $07: 37: 07.14$ & $-07: 20: 38.08$ & KVNCS1.3.1 & $0.20( \pm 0.05)$ & $\cdots$ & $\cdots$ & $\cdots$ \\
\hline J0738+1742 & $0735+178$ & $07: 38: 07.40$ & $17: 42: 19.08$ & KVNCS1.0.1 & $0.58( \pm 0.13)$ & KVNCS1.0.1 & $1.07( \pm 0.30)$ & 0.424 \\
\hline J0738+1742 & $0735+178$ & 07:38:07.40 & $17: 42: 19.08$ & KVNCS1.1.2 & $0.69( \pm 0.14)$ & $\cdots$ & $\ldots$ & 0.424 \\
\hline J0738-0626 & 0736-063 & 07:38:57.19 & $-06: 26: 58.06$ & KVNCS1.2.2 & $0.51( \pm 0.08)$ & $\cdots$ & $\cdots$ & 1.914 \\
\hline J0739+0137 & $0736+017$ & 07:39:18.04 & 01:37:04.62 & KVNCS1.0.1 & $1.20( \pm 0.10)$ & KVNCS1.0.1 & $1.50( \pm 0.34)$ & 0.189 \\
\hline J0739+0137 & $0736+017$ & 07:39:18.04 & 01:37:04.62 & KVNCS1.3.2 & $1.14( \pm 0.06)$ & KVNCS1.3.2 & $1.65( \pm 0.36)$ & 0.189 \\
\hline J0740-2444 & $0738-246$ & $07: 40: 14.70$ & $-24: 44: 36.60$ & KVNCS1.3.1 & $0.25( \pm 0.02)$ & $\cdots$ & $\cdots$ & $\cdots$ \\
\hline $\mathrm{J} 0740+2852$ & $0737+289$ & $07: 40: 33.53$ & $28: 52: 47.28$ & KVNCS1.3.2 & $0.21( \pm 0.02)$ & $\cdots$ & $\cdots$ & 0.711 \\
\hline $\mathrm{J} 0741+3112$ & $0738+313$ & $07: 41: 10.72$ & $31: 12: 00.36$ & KVNCS1.0.1 & $0.95( \pm 0.14)$ & $\cdots$ & $\cdots$ & 0.632 \\
\hline $\mathrm{J} 0741+3112$ & $0738+313$ & $07: 41: 10.72$ & $31: 12: 00.36$ & KVNCS1.1.1 & $1.00( \pm 0.09)$ & $\cdots$ & $\cdots$ & 0.632 \\
\hline J0741+2706 & $0738+272$ & $07: 41: 25.73$ & $27: 06: 45.36$ & KVNCS1.3.2 & $0.22( \pm 0.01)$ & $\cdots$ & $\cdots$ & $0.772 *$ \\
\hline J0742+4900 & $0738+491$ & $07: 42: 02.74$ & 49:00:15.48 & KVNCS1.2.1 & $0.31( \pm 0.06)$ & $\ldots$ & $\cdots$ & 2.318 \\
\hline J0742+5444 & $0738+548$ & $07: 42: 39.78$ & $54: 44: 24.72$ & KVNCS1.3.1 & $0.33( \pm 0.03)$ & KVNCS1.3.1 & $0.48( \pm 0.08)$ & 0.720 \\
\hline
\end{tabular}


Table 2 (continued)

\begin{tabular}{|c|c|c|c|c|c|c|c|c|}
\hline $\begin{array}{l}\text { IAU name } \\
(\mathrm{J} 2000) \\
(1) \\
\end{array}$ & IVS name & $\begin{array}{c}\text { R.A. } \\
\text { [hh:mm:ss] } \\
(3)\end{array}$ & $\begin{array}{c}\text { Dec. } \\
{\left[{ }^{\circ}:^{\prime}:^{\prime \prime}\right]} \\
(4) \\
\end{array}$ & Obs. code & $\begin{array}{c}\text { Flux density }( \pm 1 \sigma) \\
{[\mathrm{Jy}]} \\
(6) \\
\end{array}$ & Obs. code & $\begin{array}{c}\text { Flux density }( \pm 1 \sigma) \\
{[\mathrm{Jy}]} \\
(8) \\
\end{array}$ & Redshift (z) \\
\hline J0743+3941 & $0739+398$ & 07:43:09.88 & $39: 41: 30.84$ & KVNCS1.3.1 & $0.21( \pm 0.02)$ & $\cdots$ & $\cdots$ & 1.705 \\
\hline J0743-0440 & 0741-045 & 07:43:52.39 & $-04: 40: 20.53$ & KVNCS1.2.2 & $0.22( \pm 0.04)$ & $\cdots$ & $\cdots$ & $\cdots$ \\
\hline J0745+1011 & $0742+103$ & $07: 45: 33.05$ & 10:11:12.84 & KVNCS1.0.1 & $1.07( \pm 0.09)$ & $\cdots$ & $\cdots$ & 2.624 \\
\hline J0745-0044 & 0743-006 & $07: 45: 54.07$ & $00: 44: 17.54$ & KVNCS1.0.1 & $1.17( \pm 0.15)$ & $\cdots$ & $\cdots$ & 0.994 \\
\hline J0745-0044 & 0743-006 & $07: 45: 54.07$ & $00: 44: 17.54$ & KVNCS1.1.2 & $1.16( \pm 0.12)$ & KVNCS1.1.2 & $1.26( \pm 0.26)$ & 0.994 \\
\hline $\mathrm{J} 0745+3313$ & $0742+333$ & $07: 45: 59.33$ & $33: 13: 34.32$ & KVNCS1.2.2 & $0.18( \pm 0.04)$ & $\ldots$ & $\ldots$ & 0.611 \\
\hline J0746+2549 & $0743+259$ & $07: 46: 25.86$ & $25: 49: 02.28$ & KVNCS1.2.1 & $0.47( \pm 0.08)$ & $\cdots$ & $\cdots$ & 2.987 \\
\hline J0746+2734 & $0743+277$ & $07: 46: 40.44$ & $27: 34: 59.16$ & KVNCS1.2.1 & $0.67( \pm 0.08)$ & $\cdots$ & $\cdots$ & $2.625^{\dagger}$ \\
\hline J0748+2400 & $0745+241$ & $07: 48: 36.11$ & $24: 00: 24.12$ & KVNCS1.0.1 & $1.18( \pm 0.12)$ & KVNCS1.0.1 & $1.26( \pm 0.32)$ & 0.410 \\
\hline J0749+7420 & $0743+744$ & $07: 49: 22.44$ & $74: 20: 41.64$ & KVNCS1.3.1 & $0.25( \pm 0.03)$ & $\cdots$ & $\ldots$ & 1.629 \\
\hline $\mathrm{J} 0750+1823$ & $0747+185$ & 07:50:00.35 & 18:23:11.40 & KVNCS1.2.1 & $0.96( \pm 0.12)$ & $\cdots$ & $\cdots$ & 1.163 \\
\hline J0750+4814 & $0746+483$ & $07: 50: 20.44$ & $48: 14: 53.52$ & KVNCS1.2.1 & $0.63( \pm 0.09)$ & $\cdots$ & $\cdots$ & 1.956 \\
\hline J0750+7909 & $0742+792$ & $07: 50: 43.26$ & 79:09:16.92 & KVNCS1.3.1 & $0.25( \pm 0.03)$ & $\cdots$ & $\ldots$ & $\cdots$ \\
\hline $\mathrm{J} 0750+1231$ & $0748+126$ & 07:50:52.04 & $12: 31: 04.80$ & KVNCS1.0.1 & $4.48( \pm 0.24)$ & KVNCS1.0.1 & $3.89( \pm 0.73)$ & 0.889 \\
\hline $\mathrm{J} 0750+1231$ & $0748+126$ & $07: 50: 52.04$ & $12: 31: 04.80$ & KVNCS1.3.2 & $3.81( \pm 0.35)$ & $\ldots$ & $\ldots$ & 0.889 \\
\hline $\mathrm{J} 0750+8241$ & $0740+828$ & $07: 50: 57.77$ & $82: 41: 58.20$ & KVNCS1.3.1 & $0.36( \pm 0.03)$ & KVNCS1.3.1 & $0.36( \pm 0.06)$ & 1.991 \\
\hline $\mathrm{J} 0752+3730$ & $0749+376$ & $07: 52: 40.91$ & $37: 30: 24.48$ & KVNCS1.3.1 & $0.24( \pm 0.03)$ & KVNCS1.3.1 & $0.24( \pm 0.05)$ & 0.440 \\
\hline J0753+5352 & $0749+540$ & 07:53:01.39 & $53: 52: 59.52$ & KVNCS1.0.1 & $0.60( \pm 0.13)$ & $\ldots$ & $\ldots$ & 0.200 \\
\hline J0753+5352 & $0749+540$ & 07:53:01.39 & $53: 52: 59.52$ & KVNCS1.1.1 & $0.70( \pm 0.06)$ & $\cdots$ & $\cdots$ & 0.200 \\
\hline J0800-2302 & $0758-229$ & 08:00:12.78 & $-23: 02: 50.64$ & KVNCS1.2.2 & $0.32( \pm 0.05)$ & $\ldots$ & $\ldots$ & $\cdots$ \\
\hline J0801-2831 & $0759-283$ & 08:01:46.49 & $-28: 31: 06.96$ & KVNCS1.3.1 & $0.14( \pm 0.03)$ & $\ldots$ & $\cdots$ & $\cdots$ \\
\hline J0802+1809 & $0759+183$ & 08:02:48.05 & 18:09:49.32 & KVNCS1.2.2 & $0.39( \pm 0.04)$ & KVNCS1.2.2 & $0.26( \pm 0.08)$ & 1.586 \\
\hline J0803+0421 & $0801+044$ & 08:03:56.45 & 04:21:02.74 & KVNCS1.3.1 & $0.14( \pm 0.02)$ & $\ldots$ & $\ldots$ & $\cdots$ \\
\hline J0804-2749 & $0802-276$ & 08:04:51.46 & $-27: 49: 11.28$ & KVNCS1.2.2 & $0.38( \pm 0.06)$ & $\ldots$ & $\cdots$ & $\cdots$ \\
\hline J0805-0111 & 0802-010 & 08:05:12.88 & $-01: 11: 13.81$ & KVNCS1.2.2 & $0.40( \pm 0.06)$ & KVNCS1.2.2 & $0.31( \pm 0.07)$ & 1.388 \\
\hline J0805+6144 & $0800+618$ & 08:05:18.17 & $61: 44: 23.64$ & KVNCS1.0.1 & $0.43( \pm 0.11)$ & $\ldots$ & $\ldots$ & 3.033 \\
\hline J0805+6144 & $0800+618$ & $08: 05: 18.17$ & $61: 44: 23.64$ & KVNCS1.1.1 & $0.45( \pm 0.08)$ & $\cdots$ & $\cdots$ & 3.033 \\
\hline J0806-2652 & 0804-267 & 08:06:12.71 & $-26: 52: 33.24$ & KVNCS1.2.2 & $0.44( \pm 0.06)$ & $\cdots$ & $\cdots$ & $\ldots$ \\
\hline J0806+4504 & $0803+452$ & 08:06:33.48 & $45: 04: 32.16$ & KVNCS1.2.1 & $0.34( \pm 0.07)$ & $\cdots$ & $\cdots$ & 2.102 \\
\hline J0807-0541 & 0804-055 & 08:07:09.62 & $-05: 41: 13.92$ & KVNCS1.3.1 & $0.29( \pm 0.02)$ & KVNCS1.3.1 & $0.29( \pm 0.06)$ & $\cdots$ \\
\hline $\mathrm{J} 0807+1352$ & $0804+140$ & 08:07:38.50 & $13: 52: 17.40$ & KVNCS1.2.2 & $0.18( \pm 0.05)$ & $\cdots$ & $\ldots$ & 1.206 \\
\hline J0807+0432 & $0805+046$ & $08: 07: 57.54$ & $04: 32: 34.55$ & KVNCS1.3.1 & $0.20( \pm 0.02)$ & $\cdots$ & $\cdots$ & 2.877 \\
\hline J0808-0751 & 0805-077 & 08:08:15.54 & $-07: 51: 09.90$ & KVNCS1.0.1 & $2.45( \pm 0.16)$ & KVNCS1.0.1 & $2.26( \pm 0.49)$ & 1.837 \\
\hline J0808-0751 & 0805-077 & 08:08:15.54 & $-07: 51: 09.90$ & KVNCS1.3.2 & $1.63( \pm 0.09)$ & KVNCS1.3.2 & $1.37( \pm 0.26)$ & 1.837 \\
\hline J0808+7315 & $0802+733$ & 08:08:16.48 & $73: 15: 11.88$ & KVNCS1.3.1 & $0.31( \pm 0.03)$ & KVNCS1.3.1 & $0.28( \pm 0.04)$ & 0.496 \\
\hline J0808+4950 & $0804+499$ & 08:08:39.66 & 49:50:36.60 & KVNCS1.0.1 & $0.54( \pm 0.09)$ & $\cdots$ & $\ldots$ & 1.435 \\
\hline J0808+4950 & $0804+499$ & 08:08:39.66 & 49:50:36.60 & KVNCS1.1.1 & $0.54( \pm 0.08)$ & $\cdots$ & $\cdots$ & 1.435 \\
\hline
\end{tabular}


Table 2 (continued)

\begin{tabular}{|c|c|c|c|c|c|c|c|c|}
\hline $\begin{array}{l}\text { IAU name } \\
(\mathrm{J} 2000) \\
(1) \\
\end{array}$ & IVS name & $\begin{array}{c}\text { R.A. } \\
\text { [hh:mm:ss] } \\
(3)\end{array}$ & $\begin{array}{c}\text { Dec. } \\
{\left[{ }^{\circ}:^{\prime}:^{\prime \prime}\right]} \\
(4) \\
\end{array}$ & Obs. code & $\begin{array}{c}\text { Flux density }( \pm 1 \sigma) \\
{[\mathrm{Jy}]} \\
(6) \\
\end{array}$ & Obs. code & $\begin{array}{c}\text { Flux density }( \pm 1 \sigma) \\
{\left[\begin{array}{c}\mathrm{Jy}] \\
(8)\end{array}\right.} \\
\end{array}$ & Redshift (z) \\
\hline J0808+4052 & $0805+410$ & 08:08:56.65 & $40: 52: 44.76$ & KVNCS1.0.1 & $0.65( \pm 0.09)$ & KVNCS1.0.1 & $0.89( \pm 0.29)$ & 1.420 \\
\hline J0809+5341 & $0805+538$ & 08:09:41.72 & $53: 41: 25.08$ & KVNCS1.1.1 & $0.28( \pm 0.06)$ & $\cdots$ & $\cdots$ & 2.133 \\
\hline J0809+5341 & $0805+538$ & 08:09:41.72 & $53: 41: 25.08$ & KVNCS1.1.2 & $0.45( \pm 0.07)$ & KVNCS1.1.2 & $0.87( \pm 0.27)$ & 2.133 \\
\hline J0811+0146 & $0808+019$ & 08:11:26.70 & $01: 46: 52.21$ & KVNCS1.1.2 & $1.08( \pm 0.13)$ & KVNCS1.1.2 & $1.02( \pm 0.20)$ & 1.148 \\
\hline J0812-1810 & 0810-180 & $08: 12: 28.51$ & $-18: 10: 42.96$ & KVNCS1.3.1 & $0.15( \pm 0.03)$ & $\ldots$ & $\ldots$ & $\cdots$ \\
\hline J0813+2542 & $0810+258$ & 08:13:03.83 & $25: 42: 11.16$ & KVNCS1.2.1 & $0.58( \pm 0.06)$ & $\cdots$ & $\cdots$ & 2.025 \\
\hline J0814+6431 & $0810+646$ & 08:14:39.19 & 64:31:22.08 & KVNCS1.1.2 & $0.21( \pm 0.07)$ & $\cdots$ & $\cdots$ & $\ldots$ \\
\hline J0814+6431 & $0810+646$ & 08:14:39.19 & $64: 31: 22.08$ & KVNCS1.2.2 & $0.14( \pm 0.03)$ & $\cdots$ & $\cdots$ & $\cdots$ \\
\hline J0817+2352 & $0814+240$ & $08: 17: 10.54$ & $23: 52: 23.88$ & KVNCS1.2.1 & $0.27( \pm 0.09)$ & $\cdots$ & $\cdots$ & 1.733 \\
\hline J0817+2352 & $0814+240$ & $08: 17: 10.54$ & $23: 52: 23.88$ & KVNCS1.3.1 & $0.25( \pm 0.03)$ & $\cdots$ & $\cdots$ & 1.733 \\
\hline J0818+0517 & $0816+054$ & $08: 18: 56.23$ & $05: 17: 37.25$ & KVNCS1.2.2 & $0.15( \pm 0.03)$ & $\cdots$ & $\cdots$ & $\ldots$ \\
\hline J0820-1258 & $0818-128$ & $08: 20: 57.44$ & $-12: 58: 59.16$ & KVNCS1.2.2 & $0.46( \pm 0.06)$ & KVNCS1.2.2 & $0.44( \pm 0.07)$ & $\cdots$ \\
\hline $\mathrm{J} 0822+4041$ & $0819+408$ & $08: 22: 57.54$ & $40: 41: 49.92$ & KVNCS1.2.1 & $0.41( \pm 0.09)$ & $\ldots$ & $\ldots$ & 0.865 \\
\hline $\mathrm{J} 0823+2223$ & $0820+225$ & $08: 23: 24.76$ & $22: 23: 03.12$ & KVNCS1.2.1 & $0.84( \pm 0.10)$ & KVNCS1.2.1 & $1.97( \pm 0.27)$ & 0.951 \\
\hline J0823+2928 & $0820+296$ & $08: 23: 41.14$ & $29: 28: 28.20$ & KVNCS1.2.1 & $0.39( \pm 0.09)$ & $\ldots$ & $\ldots$ & 2.368 \\
\hline J0824+5552 & $0820+560$ & $08: 24: 47.23$ & $55: 52: 42.60$ & KVNCS1.0.1 & $0.65( \pm 0.12)$ & $\cdots$ & $\cdots$ & 1.421 \\
\hline J0824+5552 & $0820+560$ & $08: 24: 47.23$ & $55: 52: 42.60$ & KVNCS1.1.1 & $0.70( \pm 0.11)$ & $\cdots$ & $\cdots$ & 1.421 \\
\hline J0824+3916 & $0821+394$ & $08: 24: 55.48$ & $39: 16: 41.88$ & KVNCS1.1.1 & $1.21( \pm 0.07)$ & KVNCS1.1.1 & $0.73( \pm 0.11)$ & 1.216 \\
\hline J0825+1332 & $0822+137$ & $08: 25: 11.89$ & $13: 32: 32.64$ & KVNCS1.2.2 & $0.50( \pm 0.04)$ & KVNCS1.2.2 & $0.54( \pm 0.07)$ & 1.143 \\
\hline J0827+3525 & $0824+355$ & $08: 27: 38.59$ & $35: 25: 05.16$ & KVNCS1.2.1 & $0.40( \pm 0.06)$ & $\ldots$ & $\ldots$ & $2.280 *$ \\
\hline $\mathrm{J} 0830+2410$ & $0827+243$ & 08:30:52.09 & $24: 10: 59.88$ & KVNCS1.0.1 & $1.03( \pm 0.07)$ & $\cdots$ & $\cdots$ & 0.941 \\
\hline J0831+0429 & $0829+046$ & 08:31:48.86 & 04:29:39.08 & KVNCS1.0.1 & $0.77( \pm 0.10)$ & $\cdots$ & $\cdots$ & 0.174 \\
\hline J0831+0429 & $0829+046$ & 08:31:48.86 & 04:29:39.08 & KVNCS1.1.2 & $0.79( \pm 0.12)$ & $\cdots$ & $\cdots$ & 0.174 \\
\hline J0832+1832 & $0829+187$ & $08: 32: 16.04$ & $18: 32: 12.12$ & KVNCS1.2.2 & $0.24( \pm 0.03)$ & $\cdots$ & $\cdots$ & 0.154 \\
\hline J0832+4913 & $0828+493$ & $08: 32: 23.21$ & $49: 13: 21.00$ & KVNCS1.2.1 & $0.46( \pm 0.06)$ & $\cdots$ & $\cdots$ & 0.548 \\
\hline J0833+1123 & $0830+115$ & $08: 33: 14.36$ & $11: 23: 36.24$ & KVNCS1.2.2 & $0.22( \pm 0.03)$ & $\cdots$ & $\cdots$ & 2.985 \\
\hline J0833+4224 & $0830+425$ & 08:33:53.89 & $42: 24: 01.80$ & KVNCS1.2.1 & $0.55( \pm 0.08)$ & $\cdots$ & $\cdots$ & 0.249 \\
\hline J0834+6019 & $0830+605$ & $08: 34: 17.54$ & $60: 19: 46.92$ & KVNCS1.3.1 & $0.23( \pm 0.02)$ & $\cdots$ & $\cdots$ & $0.717 *$ \\
\hline J0834+5534 & $0831+557$ & $08: 34: 54.91$ & $55: 34: 21.00$ & KVNCS1.2.1 & $0.91( \pm 0.07)$ & $\cdots$ & $\cdots$ & 0.241 \\
\hline J0836+2728 & $0833+276$ & $08: 36: 22.90$ & $27: 28: 52.68$ & KVNCS1.3.1 & $0.33( \pm 0.03)$ & $\cdots$ & $\cdots$ & 0.765 \\
\hline J0836-2016 & 0834-201 & $08: 36: 39.20$ & $-20: 16: 59.52$ & KVNCS1.0.1 & $1.72( \pm 0.12)$ & $\cdots$ & $\cdots$ & 2.752 \\
\hline J0837+5825 & $0833+585$ & $08: 37: 22.40$ & $58: 25: 01.92$ & KVNCS1.2.1 & $0.76( \pm 0.09)$ & $\cdots$ & $\cdots$ & 2.101 \\
\hline J0839+0319 & $0837+035$ & $08: 39: 49.21$ & 03:19:53.87 & KVNCS1.2.2 & $0.34( \pm 0.07)$ & $\cdots$ & $\cdots$ & 1.570 \\
\hline J0839+0104 & $0837+012$ & 08:39:49.61 & 01:04:26.72 & KVNCS1.2.2 & $0.33( \pm 0.07)$ & $\cdots$ & $\cdots$ & 1.123 \\
\hline $\mathrm{J} 0840+1312$ & $0838+133$ & $08: 40: 47.60$ & $13: 12: 23.40$ & KVNCS1.0.1 & $1.44( \pm 0.11)$ & $\cdots$ & $\cdots$ & 0.680 \\
\hline $\mathrm{J} 0841+7053$ & $0836+710$ & $08: 41: 24.36$ & $70: 53: 42.36$ & $\cdots$ & $\cdots$ & KVNCS1.2.2 & $2.46( \pm 0.18)$ & 2.172 \\
\hline $\mathrm{J} 0841+7053$ & $0836+710$ & $08: 41: 24.36$ & $70: 53: 42.36$ & KVNCS1.3.2 & $2.65( \pm 0.09)$ & KVNCS1.3.2 & $2.44( \pm 0.50)$ & 2.172 \\
\hline
\end{tabular}


Table 2 (continued)

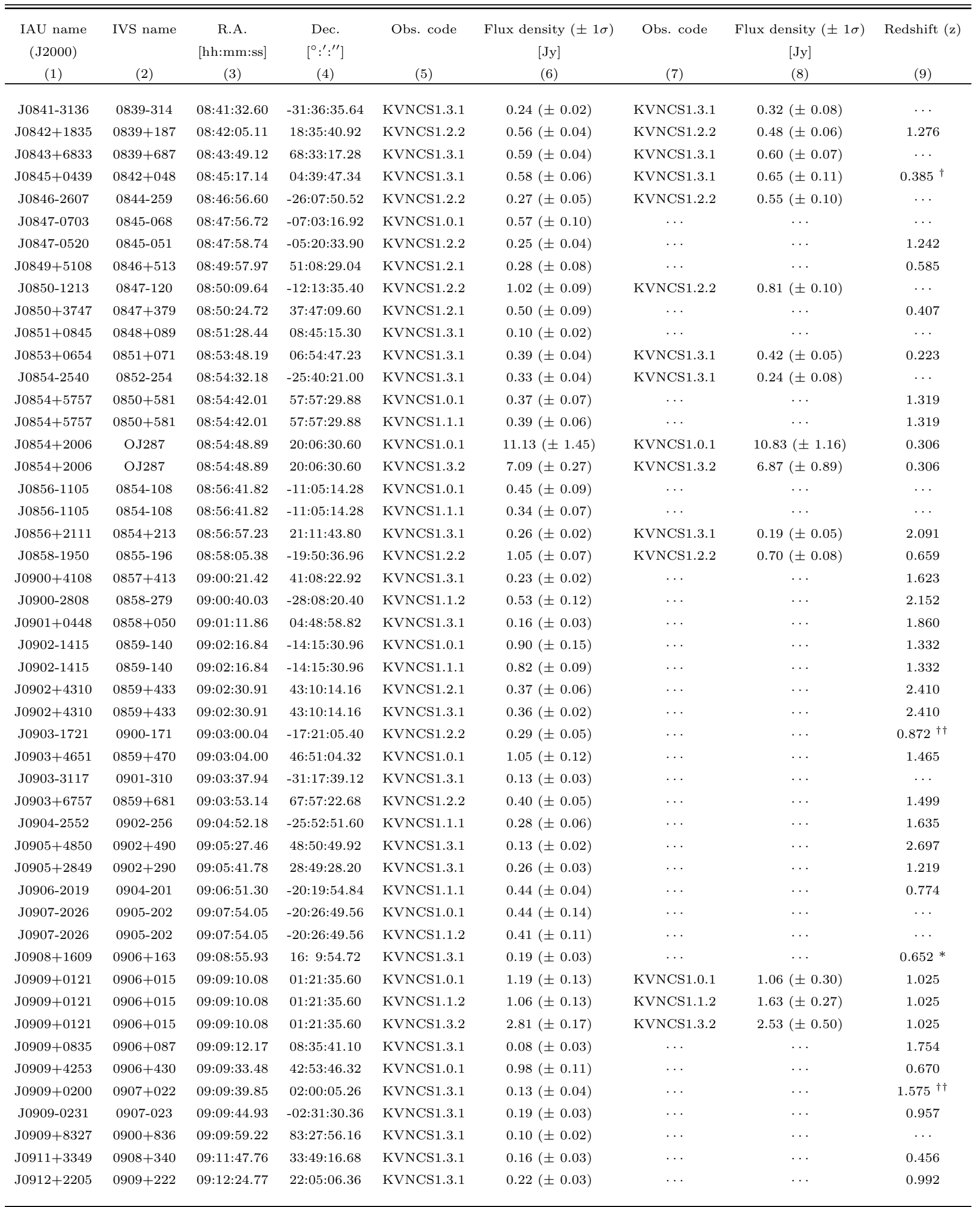


Table 2 (continued)

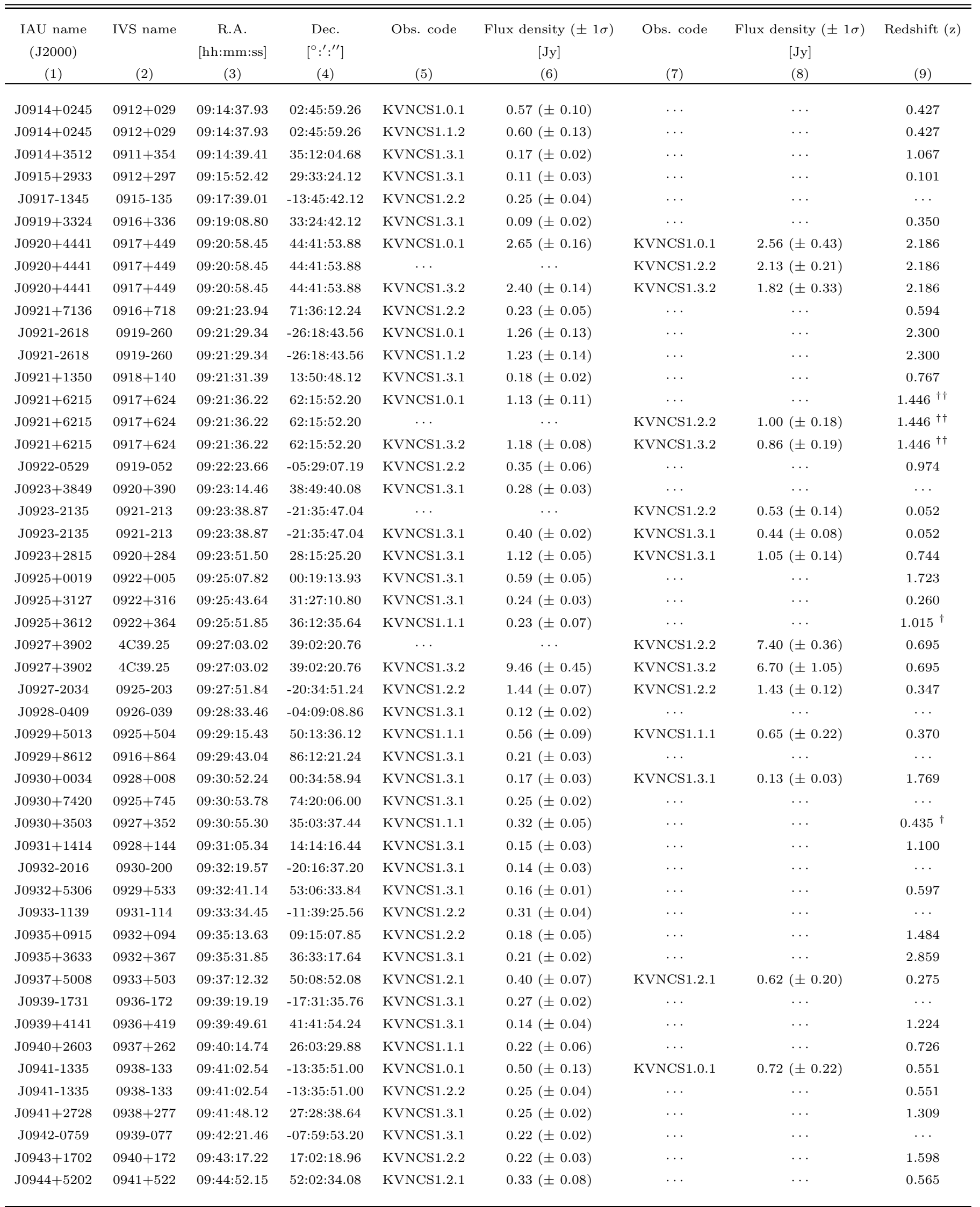


Table 2 (continued)

\begin{tabular}{|c|c|c|c|c|c|c|c|c|}
\hline $\begin{array}{l}\text { IAU name } \\
(\mathrm{J} 2000) \\
(1) \\
\end{array}$ & IVS name & $\begin{array}{c}\text { R.A. } \\
\text { [hh:mm:ss] } \\
(3)\end{array}$ & $\begin{array}{c}\text { Dec. } \\
{\left[{ }^{\circ}:^{\prime}:^{\prime \prime}\right]} \\
(4) \\
\end{array}$ & Obs. code & $\begin{array}{c}\text { Flux density }( \pm 1 \sigma) \\
{[\mathrm{Jy}]} \\
(6) \\
\end{array}$ & Obs. code & $\begin{array}{c}\text { Flux density }( \pm 1 \sigma) \\
{[\mathrm{Jy}]} \\
(8) \\
\end{array}$ & Redshift (z) \\
\hline J0945+3534 & $0942+358$ & $09: 45: 38.12$ & $35: 34: 55.20$ & KVNCS1.2.2 & $0.20( \pm 0.04)$ & $\cdots$ & $\cdots$ & 1.128 \\
\hline J0948-3223 & $0945-321$ & 09:48:09.50 & $-32: 23: 47.40$ & KVNCS1.3.1 & $0.18( \pm 0.03)$ & $\cdots$ & $\cdots$ & 2.140 \\
\hline J0948+4039 & $0945+408$ & $09: 48: 55.33$ & 40:39:44.64 & KVNCS1.0.1 & $1.61( \pm 0.14)$ & KVNCS1.0.1 & $1.18( \pm 0.26)$ & 1.250 \\
\hline J0954+2639 & $0951+268$ & 09:54:39.78 & $26: 39: 24.48$ & KVNCS1.2.2 & $0.20( \pm 0.04)$ & $\cdots$ & $\cdots$ & $\ldots$ \\
\hline J0954+7435 & $0950+748$ & $09: 54: 47.45$ & $74: 35: 57.12$ & KVNCS1.3.1 & $0.21( \pm 0.04)$ & $\cdots$ & $\cdots$ & 0.695 \\
\hline J0954+1743 & $0952+179$ & $09: 54: 56.81$ & $17: 43: 31.08$ & KVNCS1.2.2 & $0.36( \pm 0.04)$ & $\cdots$ & $\ldots$ & 1.477 \\
\hline J0956+2515 & OK290 & $09: 56: 49.88$ & $25: 15: 16.20$ & KVNCS1.0.1 & $0.96( \pm 0.11)$ & KVNCS1.0.1 & $1.18( \pm 0.30)$ & 0.707 \\
\hline J0957-1350 & 0954-135 & 09:57:18.18 & $-13: 50: 01.32$ & KVNCS1.2.2 & $0.48( \pm 0.04)$ & $\ldots$ & $\ldots$ & $\cdots$ \\
\hline J0958+6533 & $0954+658$ & $09: 58: 47.24$ & $65: 33: 54.72$ & KVNCS1.0.1 & $1.69( \pm 0.14)$ & KVNCS1.0.1 & $1.61( \pm 0.31)$ & 0.368 \\
\hline J0958+6533 & $0954+658$ & $09: 58: 47.24$ & $65: 33: 54.72$ & KVNCS1.3.2 & $1.21( \pm 0.10)$ & KVNCS1.3.2 & $1.47( \pm 0.33)$ & 0.368 \\
\hline J1000-3139 & 0958-314 & 10:00:40.68 & $-31: 39: 52.20$ & KVNCS1.2.2 & $0.40( \pm 0.05)$ & $\ldots$ & $\ldots$ & 0.009 \\
\hline $\mathrm{J} 1001+1015$ & $0959+105$ & 10:01:57.72 & $10: 15: 49.68$ & KVNCS1.3.1 & $0.13( \pm 0.03)$ & $\cdots$ & $\cdots$ & 1.533 \\
\hline $\mathrm{J} 1002+1216$ & $1000+125$ & $10: 02: 52.80$ & $12: 16: 14.52$ & KVNCS1.3.1 & $0.19( \pm 0.02)$ & $\ldots$ & $\ldots$ & 0.861 \\
\hline J1006-2159 & $1004-217$ & 10:06:46.44 & $-21: 59: 20.40$ & KVNCS1.2.2 & $0.32( \pm 0.07)$ & KVNCS1.2.2 & $0.37( \pm 0.07)$ & 0.330 \\
\hline J1006-2159 & $1004-217$ & 10:06:46.44 & $-21: 59: 20.40$ & KVNCS1.3.1 & $0.17( \pm 0.03)$ & $\ldots$ & $\ldots$ & 0.330 \\
\hline $\mathrm{J} 1007-0207$ & 1004-018 & 10:07:04.44 & $-02: 07: 10.92$ & KVNCS1.2.2 & $0.62( \pm 0.04)$ & KVNCS1.2.2 & $0.36( \pm 0.11)$ & 1.215 \\
\hline J1007-1247 & $1004-125$ & 10:07:15.24 & $-12: 47: 45.96$ & KVNCS1.3.1 & $0.21( \pm 0.03)$ & KVNCS1.3.1 & $0.15( \pm 0.05)$ & $\cdots$ \\
\hline $\mathrm{J} 1007+1356$ & $1004+141$ & 10:07:41.52 & $13: 56: 29.76$ & KVNCS1.0.1 & $0.52( \pm 0.06)$ & $\ldots$ & $\ldots$ & 2.707 \\
\hline $\mathrm{J} 1007+1356$ & $1004+141$ & 10:07:41.52 & $13: 56: 29.76$ & KVNCS1.1.2 & $0.67( \pm 0.09)$ & $\cdots$ & $\cdots$ & 2.707 \\
\hline $\mathrm{J} 1013+2449$ & $1011+250$ & $10: 13: 53.40$ & $24: 49: 16.32$ & KVNCS1.0.1 & $0.58( \pm 0.09)$ & $\cdots$ & $\cdots$ & 1.637 \\
\hline $\mathrm{J} 1013+2449$ & $1011+250$ & 10:13:53.40 & $24: 49: 16.32$ & KVNCS1.1.1 & $0.63( \pm 0.06)$ & $\cdots$ & $\cdots$ & 1.637 \\
\hline $\mathrm{J} 1014+2301$ & $1012+232$ & $10: 14: 47.04$ & 23:01:16.68 & KVNCS1.0.1 & $0.84( \pm 0.13)$ & $\cdots$ & $\cdots$ & 0.566 \\
\hline J1014+2301 & $1012+232$ & 10:14:47.04 & 23:01:16.68 & KVNCS1.1.1 & $0.73( \pm 0.07)$ & $\cdots$ & $\cdots$ & 0.566 \\
\hline J1015+4926 & $1011+496$ & 10:15:04.32 & $49: 26: 00.60$ & KVNCS1.3.1 & $0.26( \pm 0.03)$ & $\cdots$ & $\cdots$ & 0.212 \\
\hline $\mathrm{J} 1015+1227$ & $1013+127$ & $10: 15: 43.92$ & $12: 27: 07.20$ & KVNCS1.0.1 & $0.49( \pm 0.15)$ & $\cdots$ & $\cdots$ & 0.463 \\
\hline $\mathrm{J} 1015+1227$ & $1013+127$ & $10: 15: 43.92$ & $12: 27: 07.20$ & KVNCS1.1.2 & $0.29( \pm 0.08)$ & KVNCS1.1.2 & $0.92( \pm 0.27)$ & 0.463 \\
\hline J1015+0109 & $1013+014$ & $10: 15: 56.88$ & 01: 9:13.75 & KVNCS1.2.2 & $0.17( \pm 0.04)$ & $\ldots$ & $\ldots$ & 0.779 \\
\hline J1016+0513 & $1013+054$ & 10:16:03.00 & $05: 13: 02.35$ & KVNCS1.0.1 & $0.44( \pm 0.14)$ & $\cdots$ & $\cdots$ & 1.713 \\
\hline J1016+0513 & $1013+054$ & 10:16:03.00 & 05:13:02.35 & KVNCS1.1.2 & $0.55( \pm 0.11)$ & $\cdots$ & $\cdots$ & 1.713 \\
\hline $\mathrm{J} 1017+6116$ & $1014+615$ & $10: 17: 25.80$ & $61: 16: 27.48$ & KVNCS1.2.1 & $0.29( \pm 0.09)$ & $\cdots$ & $\cdots$ & 2.801 \\
\hline $\mathrm{J} 1017+6116$ & $1014+615$ & $10: 17: 25.80$ & $61: 16: 27.48$ & KVNCS1.2.2 & $0.30( \pm 0.06)$ & $\cdots$ & $\cdots$ & 2.801 \\
\hline $\mathrm{J} 1018+3542$ & $1015+359$ & $10: 18: 11.16$ & $35: 42: 39.60$ & KVNCS1.1.1 & $0.42( \pm 0.08)$ & $\cdots$ & $\cdots$ & 1.230 \\
\hline J1018+0530 & $1015+057$ & $10: 18: 27.72$ & $05: 30: 29.95$ & KVNCS1.0.1 & $0.36( \pm 0.10)$ & $\cdots$ & $\cdots$ & 1.945 \\
\hline J1018+0530 & $1015+057$ & $10: 18: 27.72$ & 05:30:29.95 & KVNCS1.1.1 & $0.29( \pm 0.07)$ & $\cdots$ & $\cdots$ & 1.945 \\
\hline $\mathrm{J} 1022+4239$ & $1019+429$ & 10:22:13.08 & $42: 39: 25.56$ & KVNCS1.3.1 & $0.29( \pm 0.02)$ & KVNCS1.3.1 & $0.24( \pm 0.06)$ & 0.991 \\
\hline $\mathrm{J} 1023+3948$ & $1020+400$ & 10:23:11.40 & $39: 48: 15.48$ & KVNCS1.1.1 & $0.62( \pm 0.07)$ & $\cdots$ & $\cdots$ & 1.254 \\
\hline $\mathrm{J} 1024+1912$ & $1022+194$ & $10: 24: 44.64$ & $19: 12: 20.52$ & KVNCS1.0.1 & $0.42( \pm 0.09)$ & $\cdots$ & $\cdots$ & 0.828 \\
\hline
\end{tabular}


Table 2 (continued)

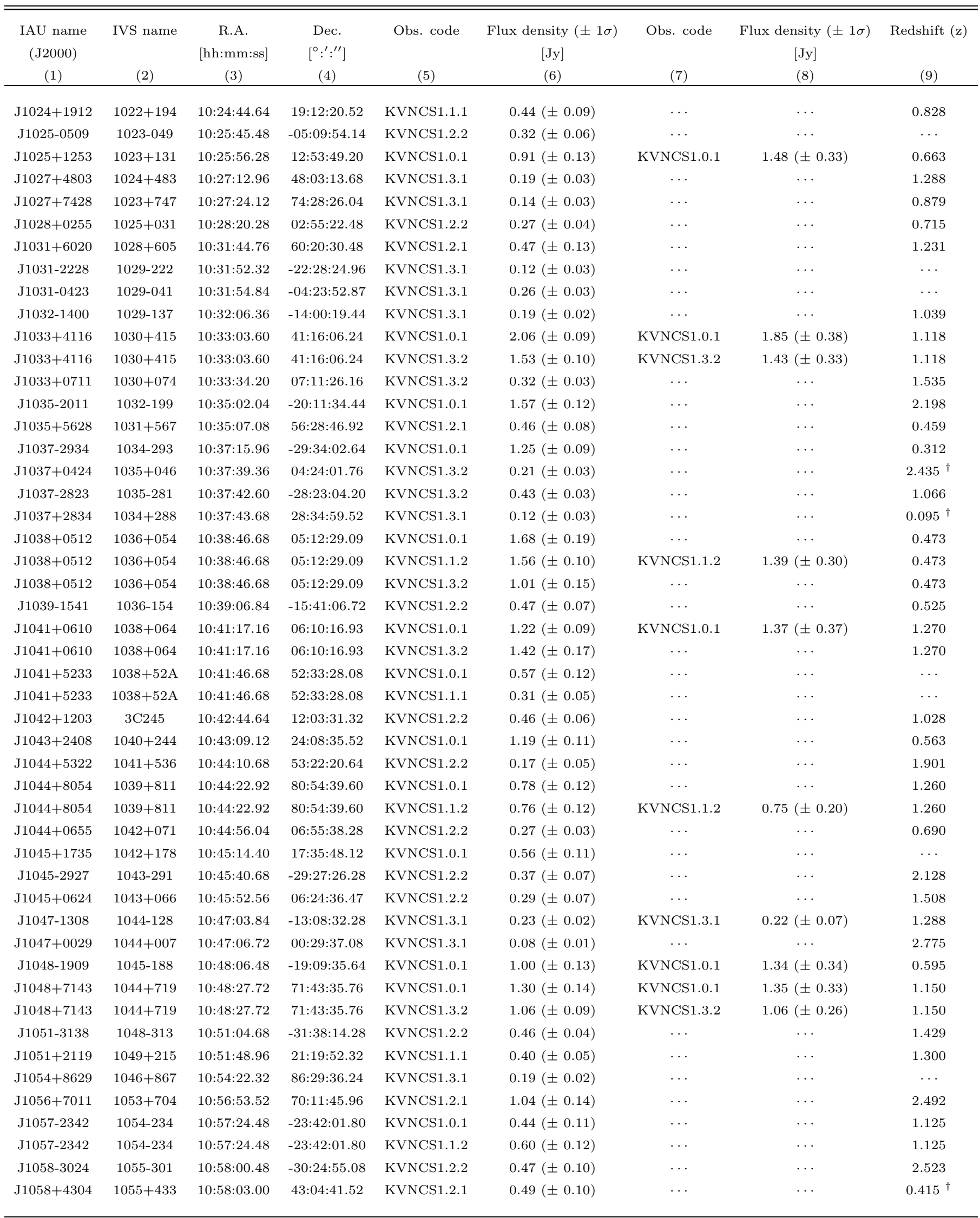


Table 2 (continued)

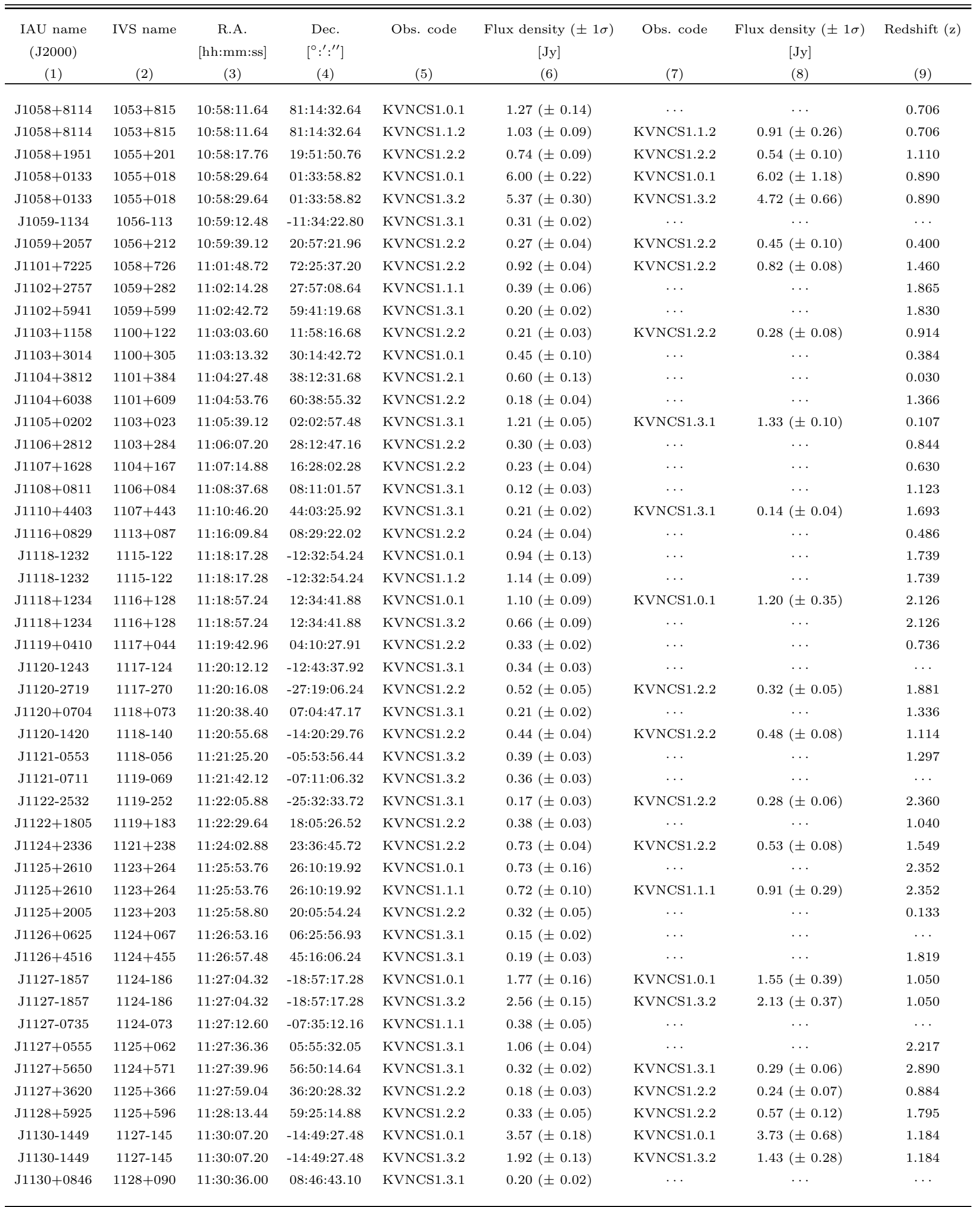


Table 2 (continued)

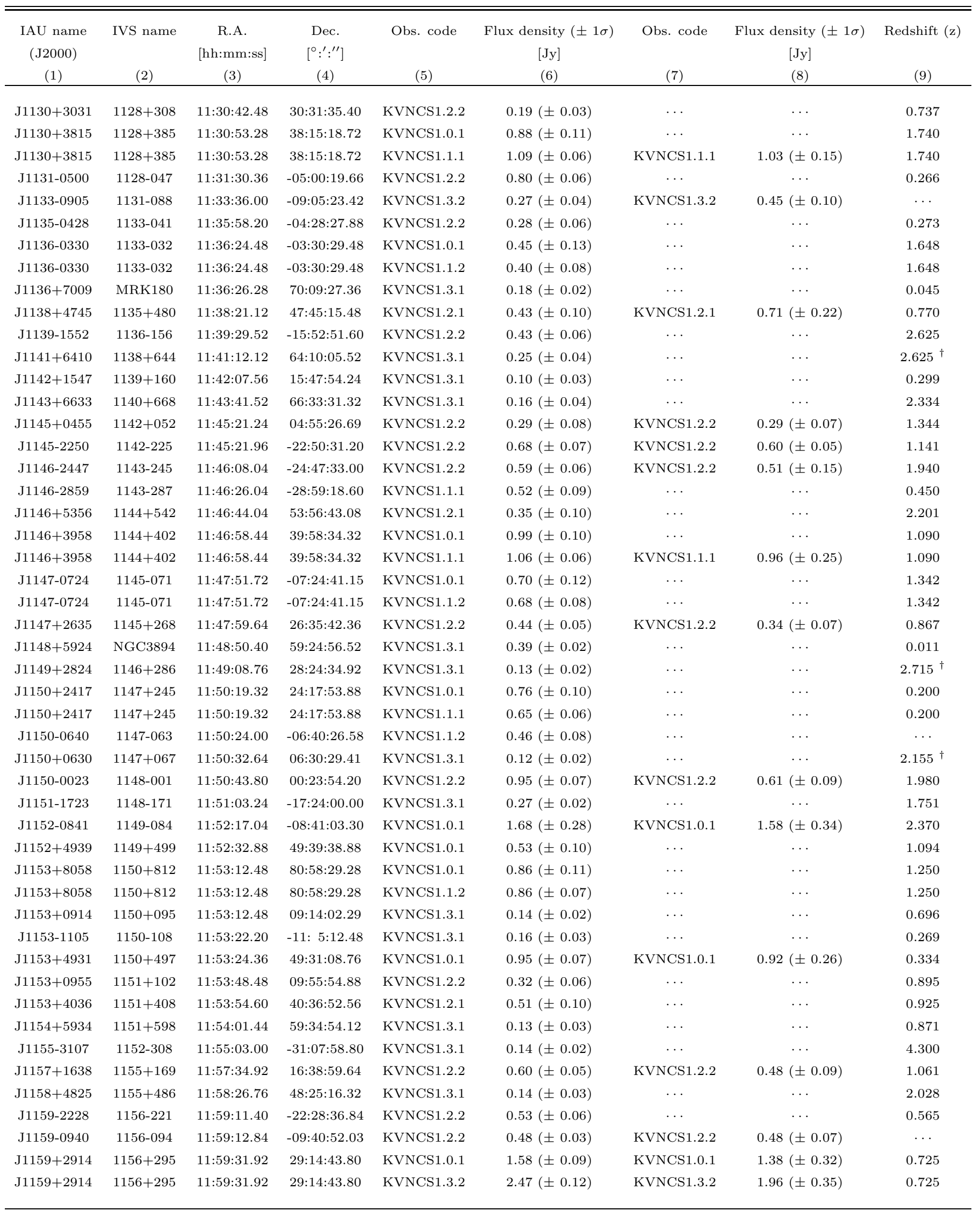


Table 2 (continued)

\begin{tabular}{|c|c|c|c|c|c|c|c|c|}
\hline J1159-2148 & $1157-215$ & 11:59:52.08 & $-21: 48: 53.64$ & KVNCS1.2.2 & $0.22( \pm 0.05)$ & KVNCS1.2.2 & $0.33( \pm 0.05)$ & 0.927 \\
\hline $\mathrm{J} 1202-0528$ & $1200-051$ & $12: 02: 34.08$ & $-05: 28: 02.50$ & KVNCS1.0.1 & $0.81( \pm 0.09)$ & $\ldots$ & $\ldots$ & 0.381 \\
\hline $\mathrm{J} 1202-0528$ & $1200-051$ & $12: 02: 34.08$ & $-05: 28: 02.50$ & KVNCS1.1.2 & $0.87( \pm 0.10)$ & KVNCS1.1.2 & $0.73( \pm 0.17)$ & 0.381 \\
\hline $\mathrm{J} 1203+4803$ & $1200+483$ & $12: 03: 29.88$ & 48:03:13.68 & KVNCS1.0.1 & $0.59( \pm 0.07)$ & $\cdots$ & $\cdots$ & 0.816 \\
\hline $\mathrm{J} 1203+4803$ & $1200+483$ & $12: 03: 29.88$ & 48:03:13.68 & KVNCS1.1.1 & $0.65( \pm 0.07)$ & $\ldots$ & $\cdots$ & 0.816 \\
\hline J1205-2634 & $1202-262$ & $12: 05: 33.36$ & $-26: 34: 04.44$ & KVNCS1.2.2 & $0.83( \pm 0.05)$ & KVNCS1.2.2 & $0.72( \pm 0.09)$ & 0.789 \\
\hline $\mathrm{J} 1206+3941$ & $1204+399$ & 12:06:37.08 & $39: 41: 03.84$ & KVNCS1.3.1 & $0.11( \pm 0.02)$ & $\ldots$ & $\ldots$ & 1.519 \\
\hline $\mathrm{J} 1206+0529$ & $1204+057$ & $12: 06: 57.96$ & $05: 29: 52.26$ & KVNCS1.2.2 & $0.22( \pm 0.05)$ & $\cdots$ & $\cdots$ & 0.791 \\
\hline J1209-2406 & $1206-238$ & 12:09:02.52 & $-24: 06: 20.88$ & KVNCS1.2.2 & $0.67( \pm 0.04)$ & KVNCS1.2.2 & $0.83( \pm 0.08)$ & 1.299 \\
\hline J1209-2032 & $1206-202$ & $12: 09: 14.76$ & $-20: 32: 39.12$ & KVNCS1.2.2 & $0.32( \pm 0.04)$ & $\ldots$ & $\ldots$ & 0.404 \\
\hline $\mathrm{J} 1209+4119$ & $1206+416$ & $12: 09: 22.68$ & $41: 19: 41.52$ & KVNCS1.3.1 & $0.14( \pm 0.03)$ & $\cdots$ & $\cdots$ & 0.377 \\
\hline $\mathrm{J} 1209+2547$ & $1207+260$ & 12:09:45.00 & $25: 47: 03.84$ & KVNCS1.3.1 & $0.20( \pm 0.04)$ & $\ldots$ & $\cdots$ & 1.435 \\
\hline $\mathrm{J} 1211+1820$ & $1208+186$ & 12:11:06.72 & $18: 20: 34.44$ & $\ldots$ & $\ldots$ & KVNCS1.2.2 & $0.31( \pm 0.10)$ & 1.515 \\
\hline $\mathrm{J} 1211+1820$ & $1208+186$ & 12:11:06.72 & $18: 20: 34.44$ & KVNCS1.3.1 & $0.21( \pm 0.03)$ & KVNCS1.3.1 & $0.35( \pm 0.05)$ & 1.515 \\
\hline $\mathrm{J} 1213-1003$ & $1210-097$ & $12: 13: 22.80$ & $-10: 03: 25.20$ & KVNCS1.3.1 & $0.14( \pm 0.03)$ & $\ldots$ & $\ldots$ & $\ldots$ \\
\hline $\mathrm{J} 1215+1654$ & $1212+171$ & $12: 15: 03.96$ & $16: 54: 37.80$ & KVNCS1.2.2 & $0.47( \pm 0.06)$ & KVNCS1.2.2 & $0.49( \pm 0.09)$ & 1.132 \\
\hline J1215-1731 & $1213-172$ & $12: 15: 46.80$ & $-17: 31: 45.48$ & KVNCS1.0.1 & $1.47( \pm 0.27)$ & KVNCS1.0.1 & $1.18( \pm 0.34)$ & $\cdots$ \\
\hline $\mathrm{J} 1215-1731$ & $1213-172$ & $12: 15: 46.80$ & $-17: 31: 45.48$ & KVNCS1.3.2 & $1.90( \pm 0.15)$ & $\ldots$ & $\ldots$ & $\ldots$ \\
\hline $\mathrm{J} 1215+3448$ & $1213+350$ & $12: 15: 55.44$ & $34: 48: 15.12$ & KVNCS1.2.2 & $0.60( \pm 0.04)$ & KVNCS1.2.2 & $0.30( \pm 0.07)$ & 0.857 \\
\hline $\mathrm{J} 1220+0203$ & $1217+023$ & $12: 20: 11.76$ & $02: 03: 42.23$ & $\mathrm{KVNCS} 1.2 .2$ & $0.25( \pm 0.05)$ & KVNCS1.2.2 & $0.36( \pm 0.08)$ & 0.240 \\
\hline $\mathrm{J} 1220+3808$ & $1218+384$ & $12: 20: 59.28$ & $38: 08: 55.68$ & KVNCS1.3.1 & $0.29( \pm 0.04)$ & $\ldots$ & $\ldots$ & 1.108 \\
\hline J1221-0241 & $1218-024$ & $12: 21: 24.12$ & $-02: 41: 49.60$ & KVNCS1.3.1 & $0.34( \pm 0.03)$ & $\cdots$ & $\cdots$ & 0.665 \\
\hline $\mathrm{J} 1221+4411$ & $1218+444$ & $12: 21: 27.00$ & $44: 11: 29.76$ & KVNCS1.3.1 & $0.27( \pm 0.02)$ & KVNCS1.3.1 & $0.18( \pm 0.06)$ & 1.347 \\
\hline $\mathrm{J} 1221+2813$ & $1219+285$ & $12: 21: 31.68$ & $28: 13: 58.44$ & KVNCS1.2.2 & $0.41( \pm 0.04)$ & KVNCS1.2.2 & $0.21( \pm 0.06)$ & 0.102 \\
\hline $\mathrm{J} 1222+0413$ & $1219+044$ & $12: 22: 22.44$ & 04:13:15.78 & KVNCS1.0.1 & $1.16( \pm 0.13)$ & KVNCS1.0.1 & $1.63( \pm 0.33)$ & 0.966 \\
\hline $\mathrm{J} 1222+0413$ & $1219+044$ & $12: 22: 22.44$ & 04:13:15.78 & KVNCS1.3.2 & $1.37( \pm 0.12)$ & KVNCS1.3.2 & $1.75( \pm 0.30)$ & 0.966 \\
\hline $\mathrm{J} 1223+8040$ & $1221+809$ & $12: 23: 40.56$ & $80: 40: 04.44$ & KVNCS1.0.1 & $0.69( \pm 0.10)$ & $\cdots$ & $\ldots$ & $\cdots$ \\
\hline $\mathrm{J} 1223+8040$ & $1221+809$ & $12: 23: 40.56$ & $80: 40: 04.44$ & KVNCS1.1.2 & $0.54( \pm 0.09)$ & $\cdots$ & $\cdots$ & $\cdots$ \\
\hline $\mathrm{J} 1224+4335$ & $1222+438$ & $12: 24: 51.48$ & $43: 35: 19.32$ & KVNCS1.3.1 & $0.31( \pm 0.02)$ & $\cdots$ & $\cdots$ & 1.075 \\
\hline $\mathrm{J} 1224+0330$ & $1222+037$ & $12: 24: 52.56$ & 03:30:50.29 & KVNCS1.1.1 & $0.36( \pm 0.05)$ & $\ldots$ & $\ldots$ & 0.955 \\
\hline $\mathrm{J} 1224+2122$ & $1222+216$ & $12: 24: 54.36$ & $21: 22: 46.56$ & KVNCS1.0.1 & $1.60( \pm 0.12)$ & KVNCS1.0.1 & $1.69( \pm 0.34)$ & 0.432 \\
\hline $\mathrm{J} 1224+2122$ & $1222+216$ & $12: 24: 54.36$ & $21: 22: 46.56$ & $\ldots$ & $\ldots$ & KVNCS1.2.2 & $2.37( \pm 0.19)$ & 0.432 \\
\hline $\mathrm{J} 1224+2122$ & $1222+216$ & $12: 24: 54.36$ & $21: 22: 46.56$ & KVNCS1.3.2 & $2.79( \pm 0.12)$ & KVNCS1.3.2 & $2.80( \pm 0.51)$ & 0.432 \\
\hline $\mathrm{J} 1225+3914$ & $1223+395$ & $12: 25: 50.52$ & $39: 14: 22.56$ & KVNCS1.3.1 & $0.14( \pm 0.03)$ & $\cdots$ & $\ldots$ & 0.623 \\
\hline $\mathrm{J} 1227+4932$ & $1225+498$ & $12: 27: 55.80$ & $49: 32: 56.04$ & KVNCS1.3.1 & $0.18( \pm 0.02)$ & $\cdots$ & $\cdots$ & 1.348 \\
\hline $\mathrm{J} 1228+3128$ & $1225+317$ & $12: 28: 24.96$ & $31: 28: 37.56$ & KVNCS1.3.1 & $0.15( \pm 0.03)$ & $\cdots$ & $\cdots$ & 2.219 \\
\hline $\mathrm{J} 1228+4858$ & $1226+492$ & $12: 28: 51.60$ & $48: 58: 01.20$ & KVNCS1.3.1 & $0.21( \pm 0.02)$ & $\cdots$ & $\cdots$ & 1.716 \\
\hline
\end{tabular}


Table 2 (continued)

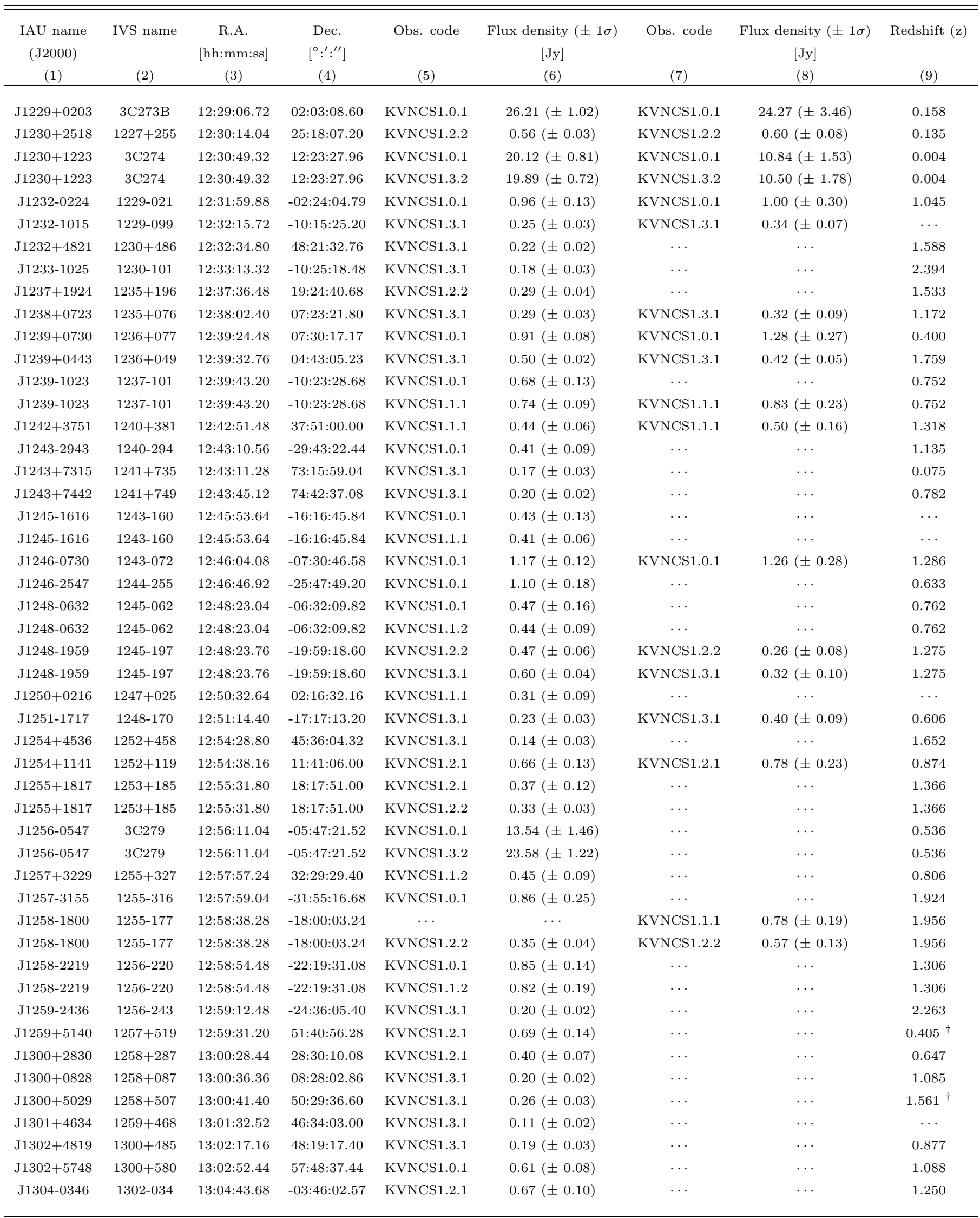


Table 2 (continued)

\begin{tabular}{|c|c|c|c|c|c|c|c|c|}
\hline $\begin{array}{l}\text { IAU name } \\
(\mathrm{J} 2000) \\
(1) \\
\end{array}$ & IVS name & $\begin{array}{c}\text { R.A. } \\
\text { [hh:mm:ss] } \\
(3)\end{array}$ & $\begin{array}{c}\text { Dec. } \\
{\left[{ }^{\circ}:^{\prime}:^{\prime \prime}\right]} \\
(4) \\
\end{array}$ & Obs. code & $\begin{array}{c}\text { Flux density }( \pm 1 \sigma) \\
{[\mathrm{Jy}]} \\
(6) \\
\end{array}$ & Obs. code & $\begin{array}{c}\text { Flux density }( \pm 1 \sigma) \\
{[\mathrm{Jy}]} \\
(8) \\
\end{array}$ & Redshift ( $\mathrm{z})$ \\
\hline $\mathrm{J} 1305-2850$ & $1302-285$ & 13:05:08.52 & $-28: 50: 42.00$ & KVNCS1.3.1 & $0.13( \pm 0.04)$ & $\cdots$ & $\cdots$ & $\cdots$ \\
\hline J1305-3132 & $1302-312$ & $13: 05: 31.20$ & $-31: 32: 52.08$ & KVNCS1.3.1 & $0.28( \pm 0.04)$ & $\cdots$ & $\cdots$ & $\cdots$ \\
\hline J1306+5529 & $1303+557$ & 13:06:03.24 & $55: 29: 43.80$ & KVNCS1.1.1 & $0.37( \pm 0.07)$ & $\cdots$ & $\cdots$ & 1.597 \\
\hline J1308+3546 & $1306+360$ & 13:08:23.64 & $35: 46: 37.20$ & KVNCS1.2.1 & $0.55( \pm 0.10)$ & $\cdots$ & $\cdots$ & 1.055 \\
\hline J1309+5557 & $1307+562$ & 13:09:09.72 & $55: 57: 38.16$ & KVNCS1.3.1 & $0.27( \pm 0.02)$ & $\cdots$ & $\cdots$ & 1.634 \\
\hline $\mathrm{J} 1309+1154$ & $1307+121$ & 13:09:33.84 & $11: 54: 24.48$ & KVNCS1.0.1 & $0.51( \pm 0.14)$ & $\ldots$ & $\ldots$ & $0.415^{\dagger}$ \\
\hline $\mathrm{J} 1309+1154$ & $1307+121$ & 13:09:33.84 & $11: 54: 24.48$ & KVNCS1.1.2 & $0.41( \pm 0.09)$ & $\cdots$ & $\cdots$ & $0.415^{\dagger}$ \\
\hline $\mathrm{J} 1310+3220$ & $1308+326$ & $13: 10: 28.56$ & $32: 20: 43.80$ & KVNCS1.0.1 & $3.27( \pm 0.18)$ & KVNCS1.0.1 & $3.39( \pm 0.48)$ & 0.998 \\
\hline $\mathrm{J} 1312+4828$ & $1310+487$ & $13: 12: 43.20$ & $48: 28: 31.08$ & KVNCS1.3.1 & $0.21( \pm 0.02)$ & $\ldots$ & $\ldots$ & 0.501 \\
\hline J1312-0424 & $1310-041$ & $13: 12: 50.76$ & $-04: 24: 49.90$ & KVNCS1.3.1 & $0.28( \pm 0.03)$ & $\cdots$ & $\cdots$ & 0.825 \\
\hline J1313-2722 & $1310-271$ & 13:13:01.56 & $-27: 22: 58.80$ & KVNCS1.3.1 & $0.17( \pm 0.02)$ & $\cdots$ & $\cdots$ & $\ldots$ \\
\hline $\mathrm{J} 1315+1220$ & $1312+126$ & $13: 15: 01.80$ & $12: 20: 52.80$ & KVNCS1.3.1 & $0.11( \pm 0.03)$ & $\ldots$ & $\ldots$ & 0.261 \\
\hline $\mathrm{J} 1317+3425$ & OP326 & $13: 17: 36.60$ & $34: 25: 15.96$ & KVNCS1.2.1 & $0.39( \pm 0.09)$ & KVNCS1.2.1 & $0.74( \pm 0.23)$ & 1.055 \\
\hline $\mathrm{J} 1317+3425$ & OP326 & $13: 17: 36.60$ & $34: 25: 15.96$ & KVNCS1.3.1 & $0.51( \pm 0.03)$ & KVNCS1.3.1 & $0.48( \pm 0.09)$ & 1.055 \\
\hline J1317-1345 & $1314-134$ & $13: 17: 36.60$ & $-13: 45: 32.76$ & KVNCS1.2.2 & $0.24( \pm 0.04)$ & $\ldots$ & $\ldots$ & $\cdots$ \\
\hline J1317-1345 & $1314-134$ & $13: 17: 36.60$ & $-13: 45: 32.76$ & KVNCS1.3.1 & $0.32( \pm 0.03)$ & $\ldots$ & $\ldots$ & $\cdots$ \\
\hline J1319-1217 & $1316-120$ & $13: 19: 12.00$ & $-12: 17: 32.28$ & KVNCS1.3.1 & $0.30( \pm 0.03)$ & KVNCS1.3.1 & $0.24( \pm 0.08)$ & 0.867 \\
\hline $\mathrm{J} 1320+0140$ & $1317+019$ & $13: 20: 26.88$ & 01:40:36.80 & KVNCS1.2.2 & $0.39( \pm 0.03)$ & $\ldots$ & $\ldots$ & 1.235 \\
\hline $\mathrm{J} 1321+2216$ & $1318+225$ & $13: 21: 11.16$ & $22: 16: 12.00$ & KVNCS1.2.1 & $0.62( \pm 0.10)$ & $\cdots$ & $\cdots$ & 0.943 \\
\hline $\mathrm{J} 1327+2210$ & $1324+224$ & $13: 27: 00.72$ & $22: 10: 50.16$ & KVNCS1.0.1 & $1.19( \pm 0.12)$ & KVNCS1.0.1 & $0.89( \pm 0.20)$ & $1.400{ }^{\dagger \dagger}$ \\
\hline $\mathrm{J} 1327+5008$ & $1325+504$ & $13: 27: 25.20$ & 50:08:49.20 & KVNCS1.3.1 & $0.12( \pm 0.03)$ & $\ldots$ & $\ldots$ & 1.012 \\
\hline $\mathrm{J} 1327+1223$ & $1325+126$ & $13: 27: 54.72$ & $12: 23: 09.24$ & KVNCS1.0.1 & $0.46( \pm 0.12)$ & $\cdots$ & $\cdots$ & 0.950 \\
\hline $\mathrm{J} 1327+1223$ & $1325+126$ & $13: 27: 54.72$ & $12: 23: 09.24$ & KVNCS1.1.2 & $0.36( \pm 0.07)$ & $\cdots$ & $\cdots$ & 0.950 \\
\hline J1329+5009 & $1327+504$ & 13:29:05.64 & 50:09:26.28 & KVNCS1.3.1 & $0.21( \pm 0.03)$ & $\cdots$ & $\cdots$ & 2.654 \\
\hline $\mathrm{J} 1329+3154$ & $1327+321$ & $13: 29: 52.80$ & $31: 54: 11.16$ & KVNCS1.0.1 & $0.78( \pm 0.14)$ & $\cdots$ & $\cdots$ & $0.335^{\dagger}$ \\
\hline J1330-3122 & $1327-311$ & 13:30:19.08 & $-31: 22: 59.16$ & KVNCS1.0.1 & $0.50( \pm 0.15)$ & $\cdots$ & $\cdots$ & 1.326 \\
\hline $\mathrm{J} 1330-3122$ & $1327-311$ & 13:30:19.08 & $-31: 22: 59.16$ & KVNCS1.1.2 & $0.55( \pm 0.11)$ & $\cdots$ & $\cdots$ & 1.326 \\
\hline $\mathrm{J} 1331+0608$ & $1329+063$ & $13: 31: 53.76$ & 06:08:23.39 & KVNCS1.3.1 & $0.09( \pm 0.02)$ & $\ldots$ & $\ldots$ & 2.533 \\
\hline J1332-0509 & $1329-049$ & $13: 32: 04.56$ & $-05: 09: 43.31$ & KVNCS1.2.1 & $1.64( \pm 0.11)$ & KVNCS1.2.1 & $1.62( \pm 0.20)$ & 2.150 \\
\hline J1332-0509 & $1329-049$ & $13: 32: 04.56$ & $-05: 09: 43.31$ & KVNCS1.3.2 & $1.58( \pm 0.16)$ & $\ldots$ & $\ldots$ & 2.150 \\
\hline $\mathrm{J} 1332-1256$ & $1329-126$ & $13: 32: 39.12$ & $-12: 56: 15.36$ & KVNCS1.2.1 & $0.43( \pm 0.08)$ & $\cdots$ & $\cdots$ & 1.492 \\
\hline J1332-1256 & $1329-126$ & $13: 32: 39.12$ & $-12: 56: 15.36$ & KVNCS1.3.1 & $0.14( \pm 0.04)$ & $\cdots$ & $\cdots$ & 1.492 \\
\hline $\mathrm{J} 1333+2725$ & $1330+276$ & 13:33:07.56 & $27: 25: 18.48$ & KVNCS1.2.1 & $0.67( \pm 0.06)$ & $\cdots$ & $\cdots$ & 2.126 \\
\hline J1333+1649 & $1331+170$ & $13: 33: 35.64$ & $16: 49: 04.08$ & KVNCS1.2.1 & $0.28( \pm 0.06)$ & $\cdots$ & $\cdots$ & 2.084 \\
\hline J1333-1950 & 1331-195 & $13: 33: 45.00$ & $-19: 50: 42.36$ & KVNCS1.2.2 & $0.29( \pm 0.05)$ & KVNCS1.2.2 & $0.37( \pm 0.09)$ & $\cdots$ \\
\hline $\mathrm{J} 1335+4542$ & $1333+459$ & $13: 35: 21.84$ & $45: 42: 38.16$ & KVNCS1.0.1 & $0.61( \pm 0.10)$ & $\cdots$ & $\cdots$ & 2.455 \\
\hline $\mathrm{J} 1335+4542$ & $1333+459$ & $13: 35: 21.84$ & $45: 42: 38.16$ & KVNCS1.1.2 & $0.61( \pm 0.10)$ & KVNCS1.1.2 & $0.92( \pm 0.30)$ & 2.455 \\
\hline
\end{tabular}


Table 2 (continued)

\begin{tabular}{|c|c|c|c|c|c|c|c|c|}
\hline $\mathrm{J} 1335+5844$ & $1333+589$ & $13: 35: 25.80$ & $58: 44: 00.24$ & KVNCS1.1.2 & $0.53( \pm 0.12)$ & $\ldots$ & $\cdots$ & $0.570^{\dagger}$ \\
\hline J1335-0511 & 1333-049 & $13: 35: 56.40$ & $-05: 11: 41.68$ & KVNCS1.3.1 & $0.13( \pm 0.02)$ & $\cdots$ & $\cdots$ & $\cdots$ \\
\hline J1336-0829 & $1333-082$ & $13: 36: 08.28$ & $-08: 29: 51.79$ & KVNCS1.0.1 & $0.68( \pm 0.12)$ & $\cdots$ & $\cdots$ & 0.023 \\
\hline J1337-1257 & $1334-127$ & $13: 37: 39.72$ & $-12: 57: 24.84$ & KVNCS1.3.2 & $3.82( \pm 0.20)$ & KVNCS1.3.2 & $3.68( \pm 0.59)$ & 0.539 \\
\hline $\mathrm{J} 1337+5501$ & $1335+552$ & $13: 37: 49.80$ & $55: 01: 02.28$ & KVNCS1.2.1 & $0.42( \pm 0.05)$ & $\ldots$ & $\ldots$ & 1.100 \\
\hline J1339-2401 & $1336-237$ & $13: 39: 01.80$ & $-24: 01: 14.16$ & KVNCS1.2.1 & $0.60( \pm 0.08)$ & KVNCS1.2.1 & $0.70( \pm 0.17)$ & 0.657 \\
\hline J1339-2620 & $1336-260$ & $13: 39: 19.80$ & $-26: 20: 30.48$ & KVNCS1.0.1 & $0.64( \pm 0.13)$ & $\ldots$ & $\ldots$ & 1.510 \\
\hline J1339-2620 & $1336-260$ & $13: 39: 19.80$ & $-26: 20: 30.48$ & KVNCS1.1.2 & $0.55( \pm 0.18)$ & $\cdots$ & $\ldots$ & 1.510 \\
\hline J1342-2900 & $1339-287$ & $13: 42: 15.48$ & $-29: 00: 41.76$ & KVNCS1.1.2 & $0.22( \pm 0.07)$ & $\cdots$ & $\cdots$ & 1.442 \\
\hline $\mathrm{J} 1344+6606$ & $1342+663$ & $13: 44: 08.52$ & $66: 06: 11.52$ & KVNCS1.1.1 & $0.29( \pm 0.04)$ & $\cdots$ & $\cdots$ & 1.351 \\
\hline J1344-1723 & $1341-171$ & $13: 44: 14.28$ & $-17: 23: 40.56$ & KVNCS1.0.1 & $0.73( \pm 0.10)$ & $\cdots$ & $\cdots$ & 2.490 \\
\hline J1344-1723 & $1341-171$ & $13: 44: 14.28$ & $-17: 23: 40.56$ & KVNCS1.1.2 & $0.67( \pm 0.14)$ & $\ldots$ & $\cdots$ & 2.490 \\
\hline $\mathrm{J} 1345+4452$ & $1343+451$ & $13: 45: 33.12$ & $44: 52: 59.52$ & KVNCS1.3.1 & $0.44( \pm 0.04)$ & KVNCS1.3.1 & $0.48( \pm 0.08)$ & 2.534 \\
\hline $\mathrm{J} 1345+0706$ & $1343+073$ & $13: 45: 49.32$ & 07:06:31.10 & KVNCS1.3.1 & $0.24( \pm 0.02)$ & $\ldots$ & $\ldots$ & 1.093 \\
\hline $\mathrm{J} 1347+1835$ & $1344+188$ & $13: 47: 23.64$ & $18: 35: 37.68$ & KVNCS1.2.1 & $0.19( \pm 0.06)$ & $\cdots$ & $\ldots$ & 2.169 \\
\hline $\mathrm{J} 1347+1835$ & $1344+188$ & $13: 47: 23.64$ & $18: 35: 37.68$ & KVNCS1.3.1 & $0.19( \pm 0.03)$ & $\ldots$ & $\ldots$ & 2.169 \\
\hline $\mathrm{J} 1347+1217$ & $1345+125$ & $13: 47: 33.36$ & $12: 17: 24.36$ & KVNCS1.2.1 & $1.32( \pm 0.09)$ & KVNCS1.2.1 & $0.97( \pm 0.24)$ & 0.122 \\
\hline $\mathrm{J} 1349+5341$ & $1347+539$ & $13: 49: 34.68$ & $53: 41: 17.16$ & KVNCS1.2.2 & $0.74( \pm 0.05)$ & KVNCS1.2.2 & $0.52( \pm 0.12)$ & 0.979 \\
\hline $\mathrm{J} 1350+0940$ & $1347+099$ & $13: 50: 22.20$ & 09:40:10.67 & KVNCS1.3.1 & $0.07( \pm 0.02)$ & $\ldots$ & $\ldots$ & 0.132 \\
\hline J1354-1041 & $1352-104$ & $13: 54: 46.44$ & $-10: 41: 02.76$ & KVNCS1.1.2 & $0.53( \pm 0.12)$ & $\ldots$ & $\ldots$ & 0.332 \\
\hline $\mathrm{J} 1357+1919$ & $1354+195$ & $13: 57: 04.32$ & 19:19:07.32 & KVNCS1.0.1 & $1.77( \pm 0.16)$ & KVNCS1.0.1 & $1.60( \pm 0.32)$ & 0.720 \\
\hline $\mathrm{J} 1357+1919$ & $1354+195$ & $13: 57: 04.32$ & 19:19:07.32 & $\ldots$ & $\ldots$ & KVNCS1.2.2 & $1.43( \pm 0.25)$ & 0.720 \\
\hline $\mathrm{J} 1357+1919$ & $1354+195$ & $13: 57: 04.32$ & 19:19:07.32 & KVNCS1.3.2 & $1.70( \pm 0.09)$ & KVNCS1.3.2 & $1.19( \pm 0.36)$ & 0.720 \\
\hline J1357-1527 & $1354-152$ & $13: 57: 11.16$ & $-15: 27: 28.80$ & KVNCS1.0.1 & $0.50( \pm 0.10)$ & $\ldots$ & $\ldots$ & 1.890 \\
\hline J1357-1527 & $1354-152$ & $13: 57: 11.16$ & $-15: 27: 28.80$ & KVNCS1.1.2 & $0.67( \pm 0.11)$ & $\cdots$ & $\cdots$ & 1.890 \\
\hline $\mathrm{J} 1357+7643$ & $1357+769$ & $13: 57: 55.44$ & $76: 43: 21.00$ & KVNCS1.1.2 & $0.60( \pm 0.12)$ & $\cdots$ & $\cdots$ & 1.585 \\
\hline $\mathrm{J} 1358+1119$ & $1355+115$ & $13: 58: 22.44$ & $11: 19: 32.88$ & KVNCS1.3.1 & $0.19( \pm 0.02)$ & $\ldots$ & $\ldots$ & $\cdots$ \\
\hline $\mathrm{J} 1359+0159$ & $1356+022$ & $13: 59: 27.24$ & 01:59:54.56 & KVNCS1.1.1 & $0.47( \pm 0.08)$ & KVNCS1.1.1 & $0.99( \pm 0.19)$ & 1.326 \\
\hline $\mathrm{J} 1359+4011$ & $1357+404$ & $13: 59: 38.04$ & 40:11:38.40 & KVNCS1.2.1 & $0.26( \pm 0.07)$ & $\ldots$ & $\ldots$ & 0.407 \\
\hline J1400-1858 & $1357-187$ & 14:00:03.96 & $-18: 58: 10.92$ & KVNCS1.2.1 & $0.25( \pm 0.08)$ & $\ldots$ & $\ldots$ & 0.114 \\
\hline $\mathrm{J} 1400-1858$ & $1357-187$ & 14:00:03.96 & $-18: 58: 10.92$ & KVNCS1.3.1 & $0.32( \pm 0.03)$ & KVNCS1.3.1 & $0.36( \pm 0.06)$ & 0.114 \\
\hline J1401-0916 & $1358-090$ & 14:01:05.16 & $-09: 16: 31.58$ & KVNCS1.3.1 & $0.33( \pm 0.03)$ & KVNCS1.3.1 & $0.31( \pm 0.04)$ & 0.667 \\
\hline J1401-3004 & $1358-298$ & 14:01:35.04 & $-30: 04: 36.84$ & KVNCS1.3.1 & $0.18( \pm 0.02)$ & $\cdots$ & $\cdots$ & 0.689 \\
\hline $\mathrm{J} 1402-2822$ & $1359-281$ & $14: 02: 02.40$ & $-28: 22: 25.32$ & KVNCS1.2.1 & $0.50( \pm 0.10)$ & $\cdots$ & $\cdots$ & 0.802 \\
\hline J1404-0013 & $1401+000$ & 14:04:12.00 & 00:13:25.09 & KVNCS1.2.1 & $0.38( \pm 0.08)$ & $\cdots$ & $\cdots$ & 1.223 \\
\hline $\mathrm{J} 1405+0415$ & $1402+044$ & 14:05:00.96 & $04: 15: 35.82$ & KVNCS1.0.1 & $0.69( \pm 0.10)$ & $\cdots$ & $\cdots$ & 3.209 \\
\hline $\mathrm{J} 1405+0415$ & $1402+044$ & 14:05:00.96 & $04: 15: 35.82$ & KVNCS1.1.2 & $0.62( \pm 0.07)$ & $\cdots$ & $\cdots$ & 3.209 \\
\hline
\end{tabular}


Table 2 (continued)

\begin{tabular}{|c|c|c|c|c|c|c|c|c|}
\hline $\begin{array}{l}\text { IAU name } \\
(\mathrm{J} 2000) \\
(1) \\
\end{array}$ & IVS name & $\begin{array}{c}\text { R.A. } \\
\text { [hh:mm:ss] } \\
(3)\end{array}$ & $\begin{array}{c}\text { Dec. } \\
{\left[{ }^{\circ}:^{\prime}:^{\prime \prime}\right]} \\
(4) \\
\end{array}$ & Obs. code & $\begin{array}{c}\text { Flux density }( \pm 1 \sigma) \\
{[\mathrm{Jy}]} \\
(6) \\
\end{array}$ & Obs. code & $\begin{array}{c}\text { Flux density }( \pm 1 \sigma) \\
{[\mathrm{Jy}]} \\
(8)\end{array}$ & Redshift (z) \\
\hline J1405+4056 & $1403+411$ & 14:05:07.80 & $40: 56: 57.84$ & KVNCS1.3.1 & $0.22( \pm 0.02)$ & $\cdots$ & $\cdots$ & 2.000 \\
\hline $\mathrm{J} 1406-0848$ & $1403-085$ & 14:06:00.72 & $-08: 48: 06.88$ & KVNCS1.2.1 & $0.30( \pm 0.08)$ & $\cdots$ & $\cdots$ & 1.763 \\
\hline J1406-0848 & $1403-085$ & 14:06:00.72 & $-08: 48: 06.88$ & KVNCS1.2.2 & $0.34( \pm 0.05)$ & $\cdots$ & $\cdots$ & 1.763 \\
\hline $\mathrm{J} 1407+2827$ & OQ208 & 14:07:00.48 & $28: 27: 14.76$ & KVNCS1.1.1 & $0.32( \pm 0.09)$ & $\cdots$ & $\cdots$ & 0.077 \\
\hline J1407-2701 & $1404-267$ & $14: 07: 29.64$ & $-27: 01: 04.44$ & KVNCS1.3.1 & $0.25( \pm 0.03)$ & $\cdots$ & $\cdots$ & 0.022 \\
\hline J1408-2900 & $1405-287$ & $14: 08: 49.56$ & $-29: 00: 23.76$ & KVNCS1.2.1 & $0.52( \pm 0.09)$ & $\cdots$ & $\cdots$ & 0.575 \\
\hline J1408-0752 & $1406-076$ & $14: 08: 56.40$ & $-07: 52: 26.65$ & KVNCS1.0.1 & $1.06( \pm 0.10)$ & $\cdots$ & $\ldots$ & 1.494 \\
\hline $\mathrm{J} 1408-0752$ & $1406-076$ & 14:08:56.40 & $-07: 52: 26.65$ & KVNCS1.1.2 & $1.06( \pm 0.10)$ & KVNCS1.1.2 & $0.87( \pm 0.19)$ & 1.494 \\
\hline $\mathrm{J} 1411+3705$ & $1409+373$ & 14:11:14.64 & $37: 05: 35.52$ & KVNCS1.3.1 & $0.28( \pm 0.03)$ & $\cdots$ & $\cdots$ & $\ldots$ \\
\hline $\mathrm{J} 1411+2134$ & $1409+218$ & 14:11:54.96 & $21: 34: 23.52$ & KVNCS1.3.1 & $0.29( \pm 0.03)$ & $\cdots$ & $\cdots$ & $0.335^{\dagger}$ \\
\hline J1415-0955 & $1412-097$ & $14: 15: 20.88$ & $-09: 55: 58.33$ & KVNCS1.3.1 & $0.26( \pm 0.03)$ & KVNCS1.3.1 & $0.26( \pm 0.05)$ & 2.001 \\
\hline $\mathrm{J} 1415+1320$ & $1413+135$ & $14: 15: 58.68$ & $13: 20: 23.64$ & KVNCS1.0.1 & $0.96( \pm 0.19)$ & KVNCS1.0.1 & $1.06( \pm 0.27)$ & 0.247 \\
\hline $\mathrm{J} 1415+1320$ & $1413+135$ & $14: 15: 58.68$ & $13: 20: 23.64$ & KVNCS1.1.2 & $0.97( \pm 0.09)$ & KVNCS1.1.2 & $0.89( \pm 0.17)$ & 0.247 \\
\hline $\mathrm{J} 1417+4607$ & $1415+463$ & $14: 17: 08.16$ & $46: 07: 05.52$ & KVNCS1.2.1 & $0.51( \pm 0.08)$ & $\ldots$ & $\ldots$ & 1.554 \\
\hline $\mathrm{J} 1419+0628$ & $1416+067$ & 14:19:08.04 & $06: 28: 34.79$ & KVNCS1.3.1 & $0.24( \pm 0.02)$ & $\cdots$ & $\cdots$ & 1.438 \\
\hline $\mathrm{J} 1419+5423$ & $1418+546$ & $14: 19: 46.56$ & $54: 23: 14.64$ & KVNCS1.0.1 & $1.37( \pm 0.13)$ & KVNCS1.0.1 & $1.04( \pm 0.28)$ & 0.153 \\
\hline $\mathrm{J} 1419+5423$ & $1418+546$ & $14: 19: 46.56$ & $54: 23: 14.64$ & KVNCS1.1.2 & $1.11( \pm 0.14)$ & KVNCS1.1.2 & $0.79( \pm 0.18)$ & 0.153 \\
\hline $\mathrm{J} 1419+3821$ & $1417+385$ & $14: 19: 46.56$ & $38: 21: 48.60$ & KVNCS1.1.2 & $0.78( \pm 0.07)$ & $\ldots$ & $\ldots$ & 1.830 \\
\hline J1419-1928 & $1417-192$ & $14: 19: 49.80$ & $-19: 28: 25.32$ & KVNCS1.2.1 & $0.42( \pm 0.06)$ & $\cdots$ & $\cdots$ & 0.120 \\
\hline $\mathrm{J} 1427+2348$ & $1424+240$ & $14: 27: 00.36$ & 23:48:00.00 & KVNCS1.3.1 & $0.25( \pm 0.02)$ & $\ldots$ & $\ldots$ & 0.160 \\
\hline $\mathrm{J} 1429+5406$ & $1427+543$ & $14: 29: 21.84$ & 54:06:11.16 & KVNCS1.2.1 & $0.30( \pm 0.06)$ & $\cdots$ & $\cdots$ & 3.013 \\
\hline $\mathrm{J} 1430+1043$ & $1427+109$ & 14:30:09.72 & $10: 43: 26.76$ & KVNCS1.0.1 & $0.69( \pm 0.15)$ & $\ldots$ & $\ldots$ & 1.707 \\
\hline $\mathrm{J} 1430+1043$ & $1427+109$ & 14:30:09.72 & $10: 43: 26.76$ & KVNCS1.1.2 & $0.65( \pm 0.12)$ & KVNCS1.1.2 & $0.46( \pm 0.13)$ & 1.707 \\
\hline $\mathrm{J} 1430+3649$ & $1428+370$ & $14: 30: 40.68$ & $36: 49: 03.72$ & KVNCS1.2.1 & $0.40( \pm 0.09)$ & $\ldots$ & $\ldots$ & 0.567 \\
\hline $\mathrm{J} 1430+3649$ & $1428+370$ & $14: 30: 40.68$ & $36: 49: 03.72$ & KVNCS1.3.1 & $0.42( \pm 0.03)$ & KVNCS1.3.1 & $0.34( \pm 0.08)$ & 0.567 \\
\hline J1432-1801 & $1430-178$ & $14: 32: 57.84$ & $-18: 01: 35.40$ & KVNCS1.2.1 & $0.47( \pm 0.08)$ & $\ldots$ & $\ldots$ & 2.331 \\
\hline J1433-1548 & $1430-155$ & $14: 33: 21.60$ & $-15: 48: 44.64$ & KVNCS1.0.1 & $0.55( \pm 0.15)$ & $\cdots$ & $\cdots$ & 1.573 \\
\hline J1433-1548 & $1430-155$ & $14: 33: 21.60$ & $-15: 48: 44.64$ & KVNCS1.1.2 & $0.53( \pm 0.12)$ & $\cdots$ & $\cdots$ & 1.573 \\
\hline $\mathrm{J} 1434+4203$ & $1432+422$ & $14: 34: 05.52$ & $42: 03: 15.84$ & KVNCS1.2.1 & $0.19( \pm 0.05)$ & $\cdots$ & $\cdots$ & 1.240 \\
\hline J1434-1146 & $1431-115$ & $14: 34: 21.00$ & $-11: 46: 19.56$ & KVNCS1.3.1 & $0.21( \pm 0.03)$ & $\cdots$ & $\cdots$ & $\cdots$ \\
\hline $\mathrm{J} 1434+1952$ & $1432+200$ & $14: 34: 39.72$ & $19: 52: 00.84$ & KVNCS1.3.1 & $0.32( \pm 0.02)$ & $\cdots$ & $\ldots$ & 1.382 \\
\hline $\mathrm{J} 1436+2321$ & $1434+235$ & $14: 36: 41.04$ & 23:21:03.24 & KVNCS1.2.1 & $0.87( \pm 0.06)$ & KVNCS1.2.1 & $0.69( \pm 0.12)$ & 1.547 \\
\hline J1436+6336 & $1435+638$ & $14: 36: 45.72$ & $63: 36: 37.80$ & KVNCS1.0.1 & $1.37( \pm 0.19)$ & $\cdots$ & $\ldots$ & $2.067 *$ \\
\hline $\mathrm{J} 1438-2204$ & $1435-218$ & 14:38:09.60 & $-22: 04: 54.84$ & KVNCS1.0.1 & $0.65( \pm 0.17)$ & $\cdots$ & $\cdots$ & 1.187 \\
\hline J1438-2204 & $1435-218$ & 14:38:09.60 & $-22: 04: 54.84$ & KVNCS1.1.2 & $1.09( \pm 0.12)$ & KVNCS1.1.2 & $1.36( \pm 0.35)$ & 1.187 \\
\hline $\mathrm{J} 1438+3710$ & $1436+373$ & $14: 38: 53.52$ & $37: 10: 35.40$ & KVNCS1.2.1 & $0.46( \pm 0.06)$ & KVNCS1.2.1 & $0.62( \pm 0.18)$ & $2.400 *$ \\
\hline $\mathrm{J} 1439+2114$ & $1436+214$ & 14:39:09.00 & $21: 14: 50.64$ & KVNCS1.3.1 & $0.13( \pm 0.03)$ & $\ldots$ & $\ldots$ & $\cdots$ \\
\hline
\end{tabular}


Table 2 (continued)

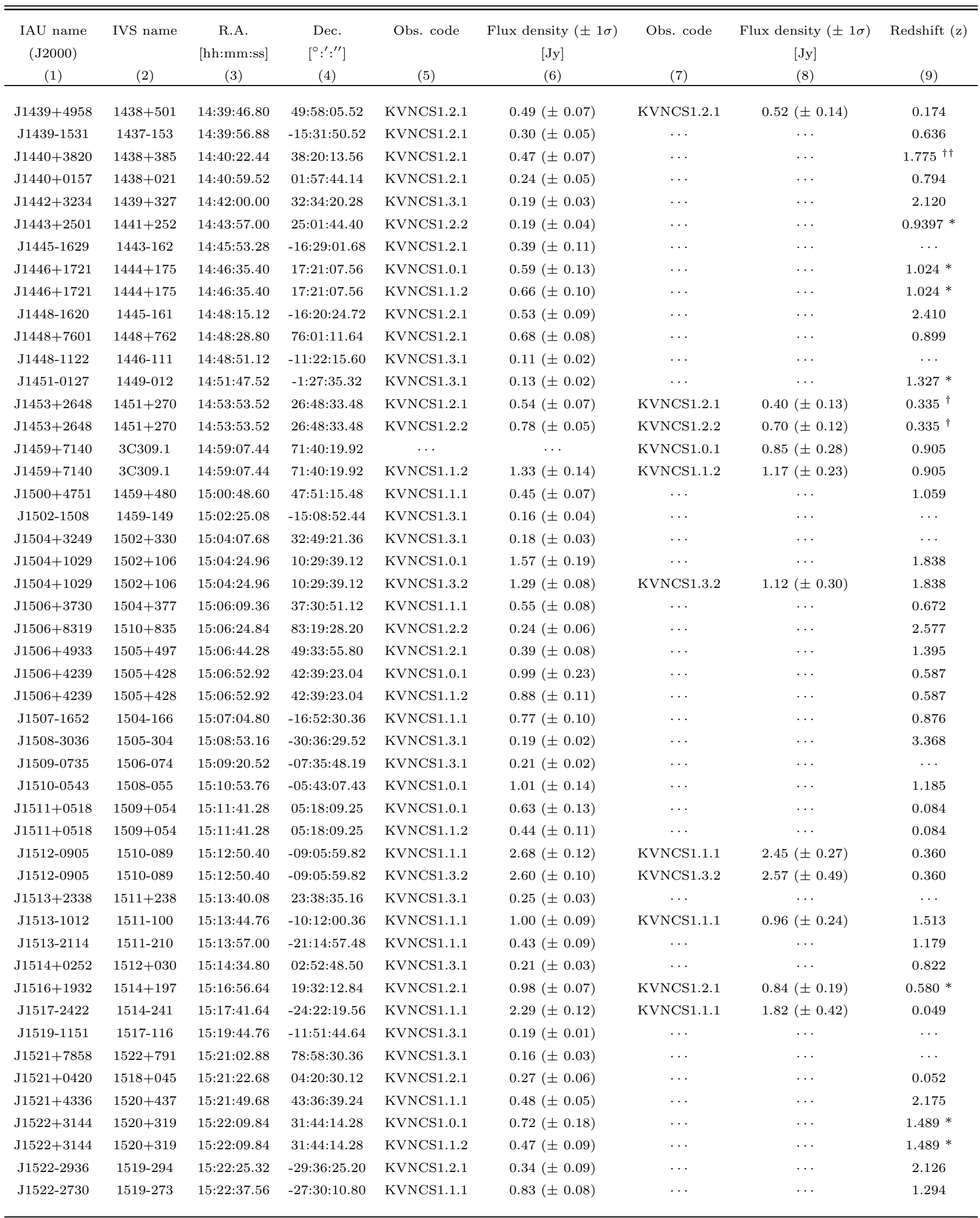


Table 2 (continued)

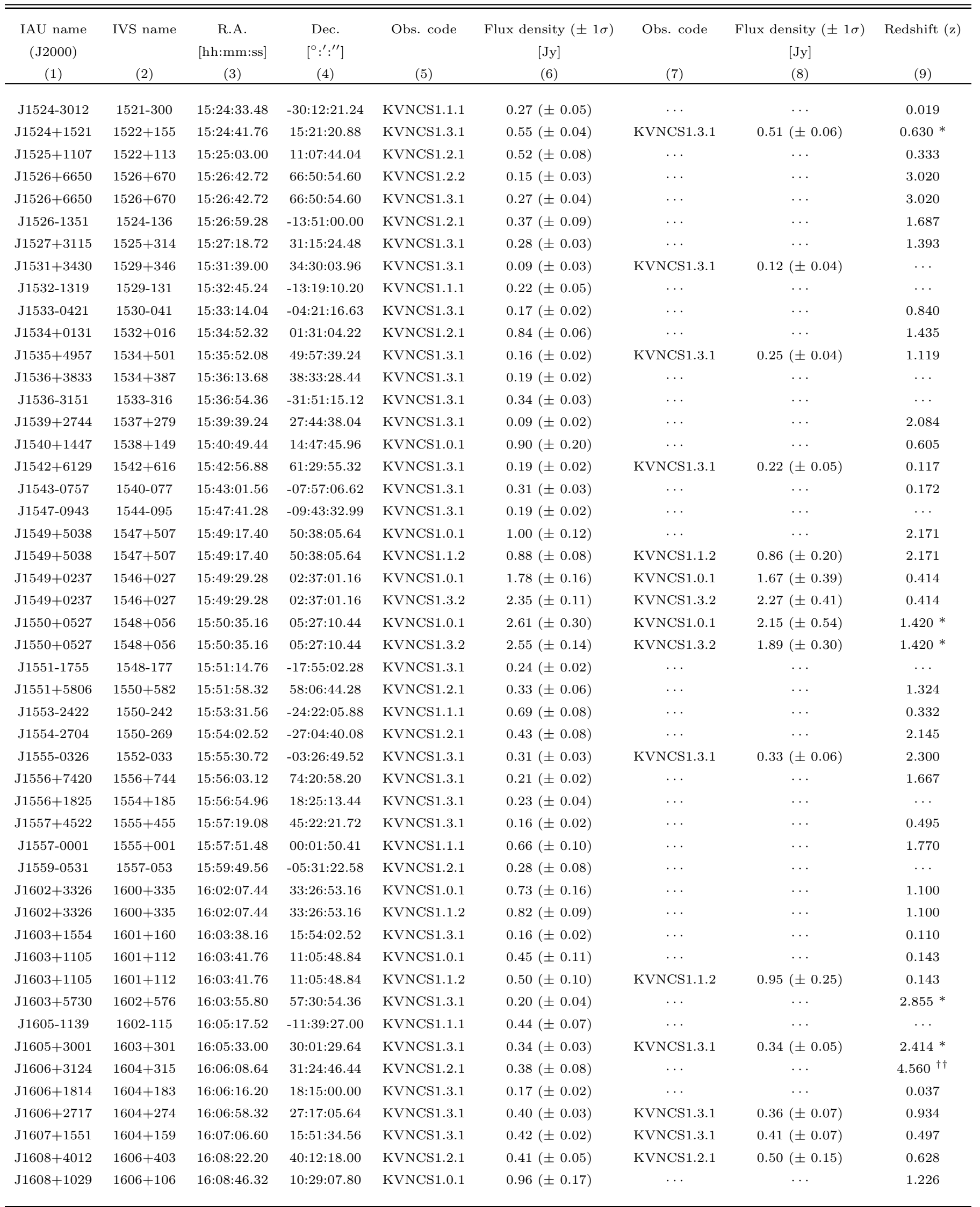


Table 2 (continued)

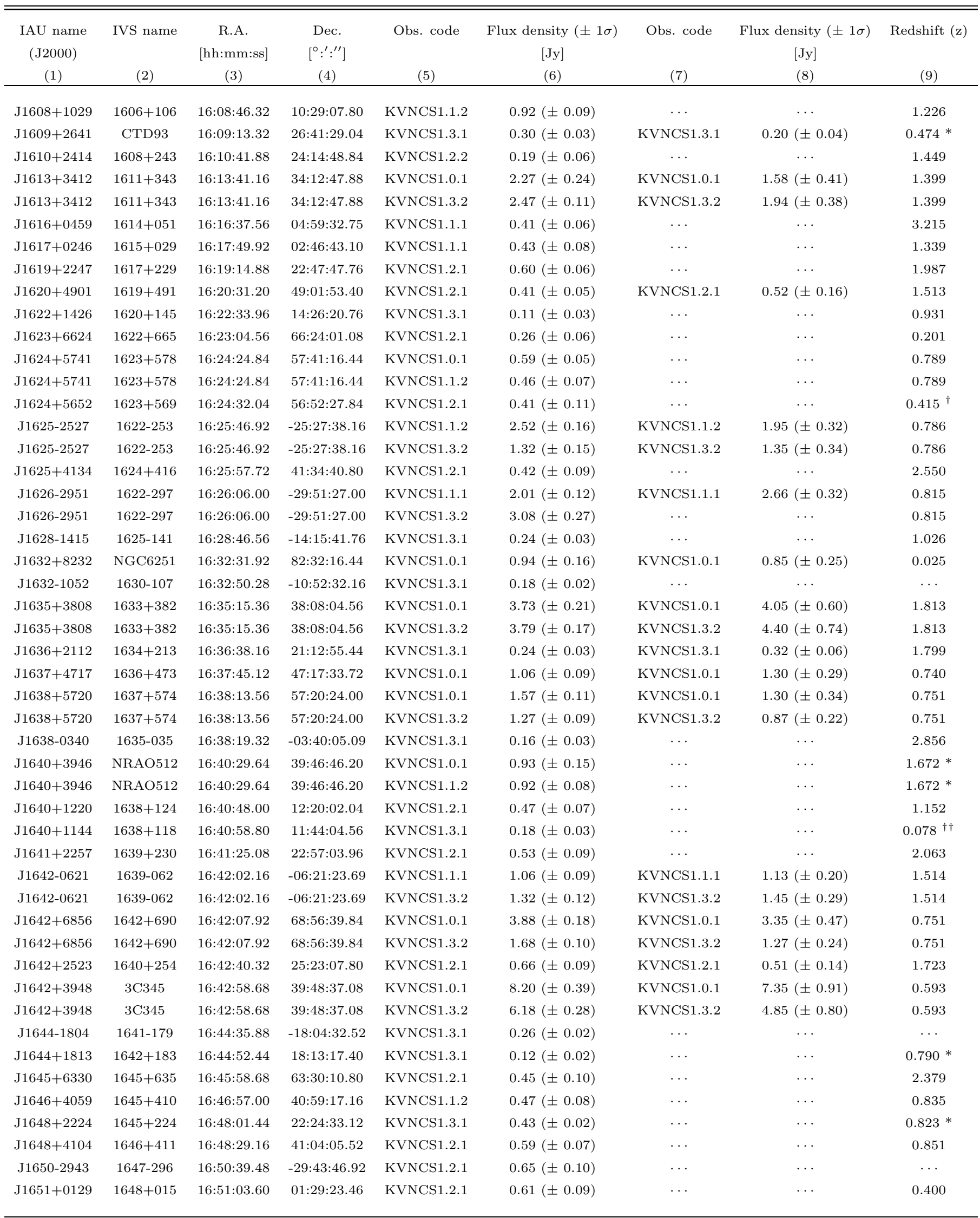


Table 2 (continued)

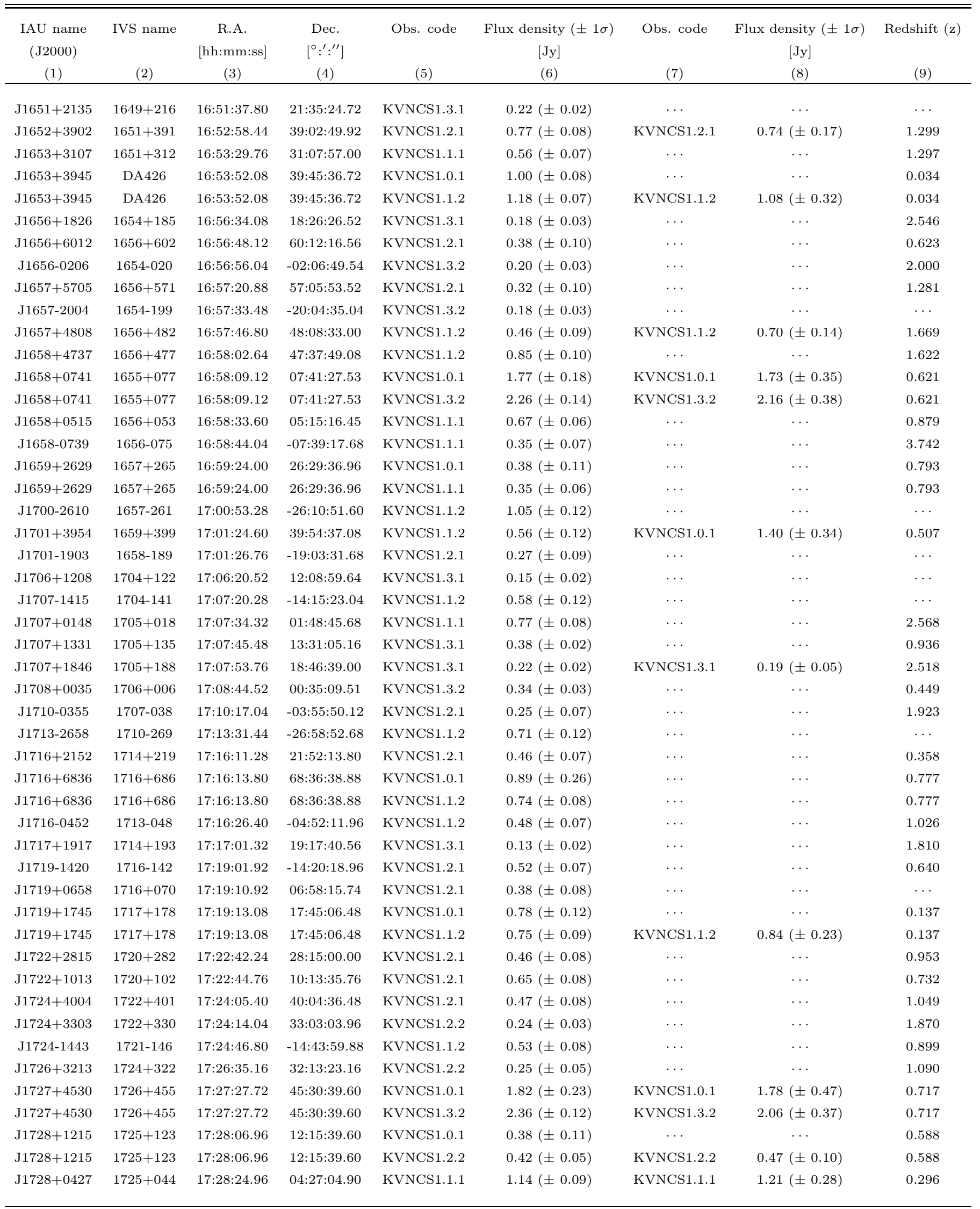


Table 2 (continued)

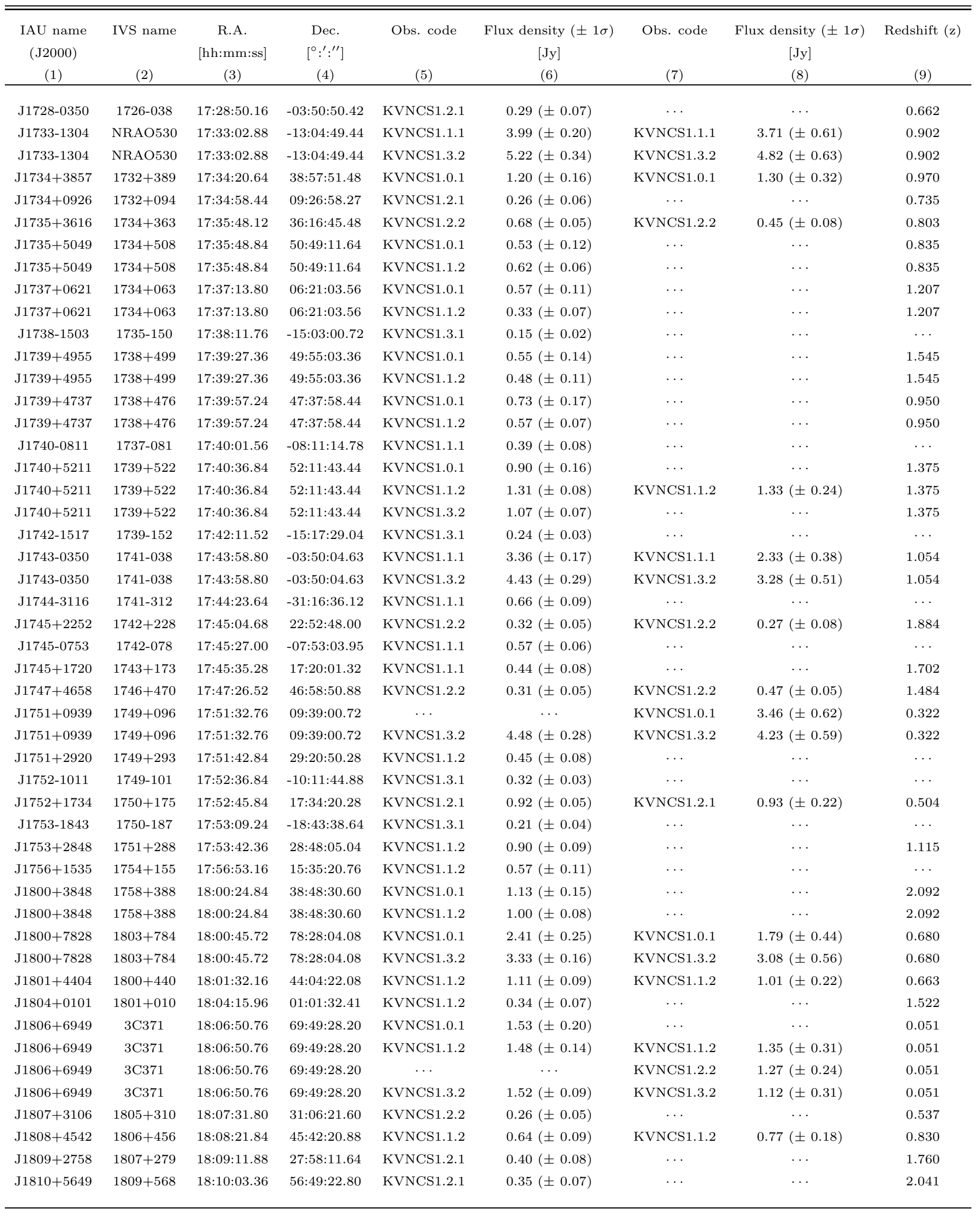


Table 2 (continued)

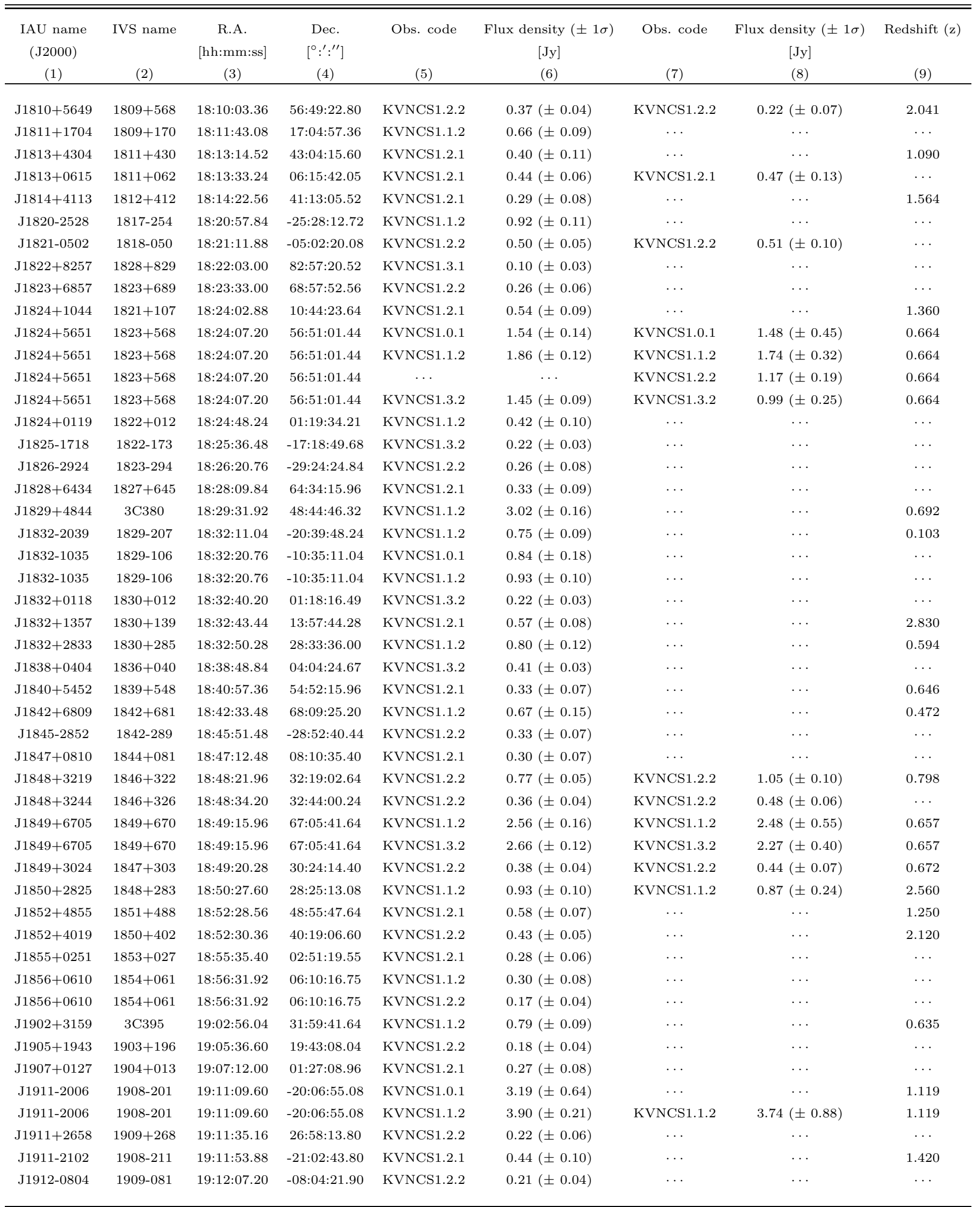


Table 2 (continued)

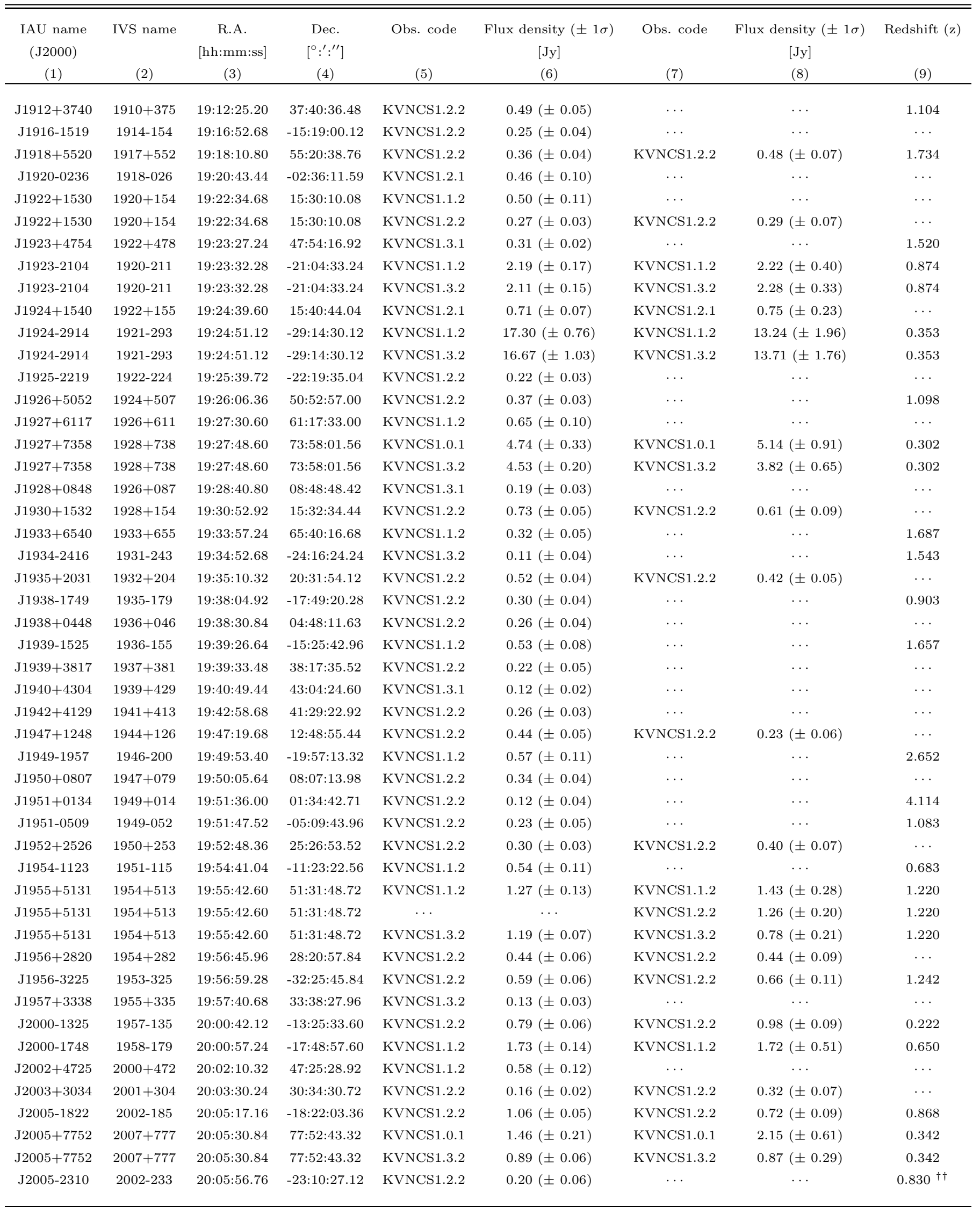


Table 2 (continued)

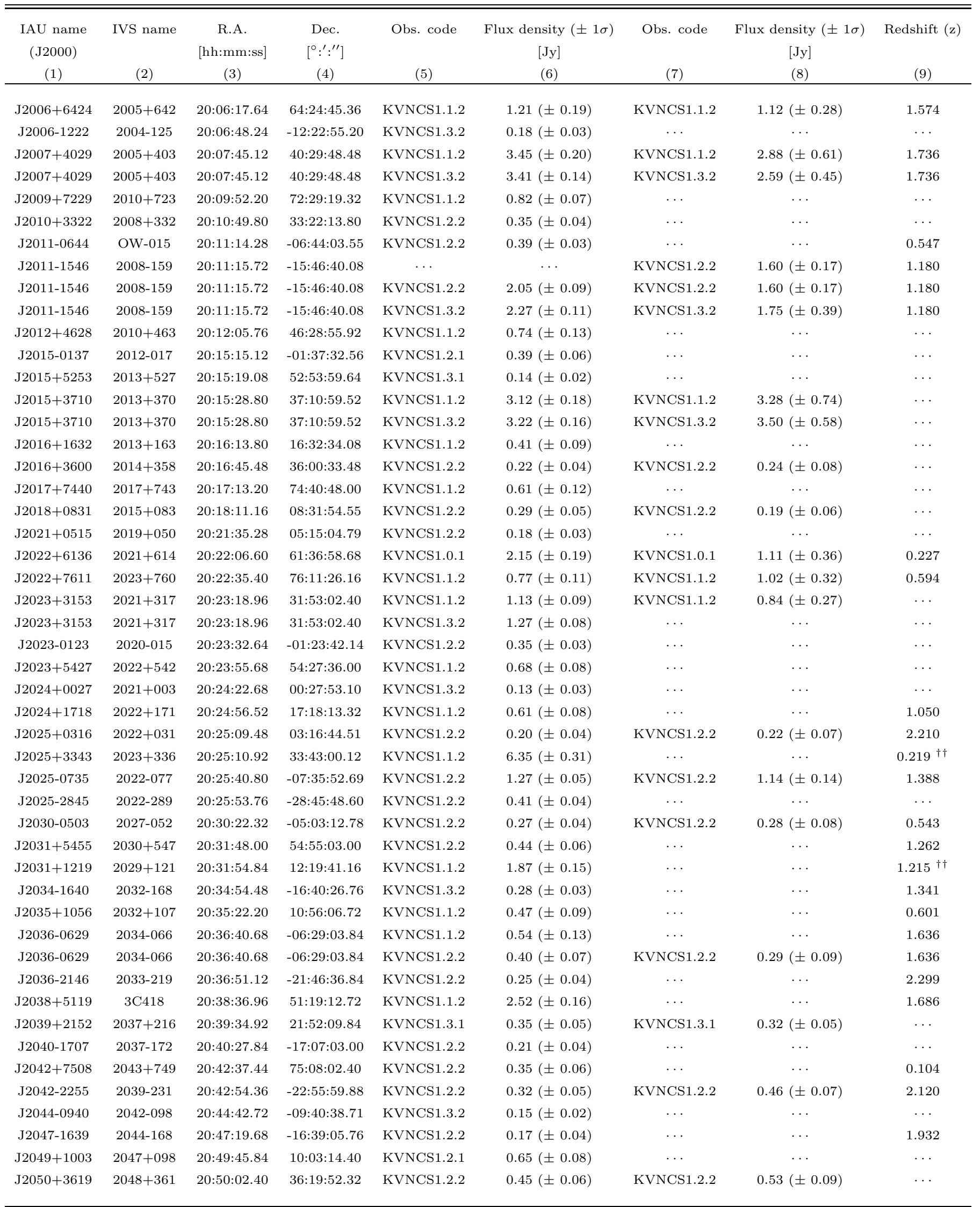


Table 2 (continued)

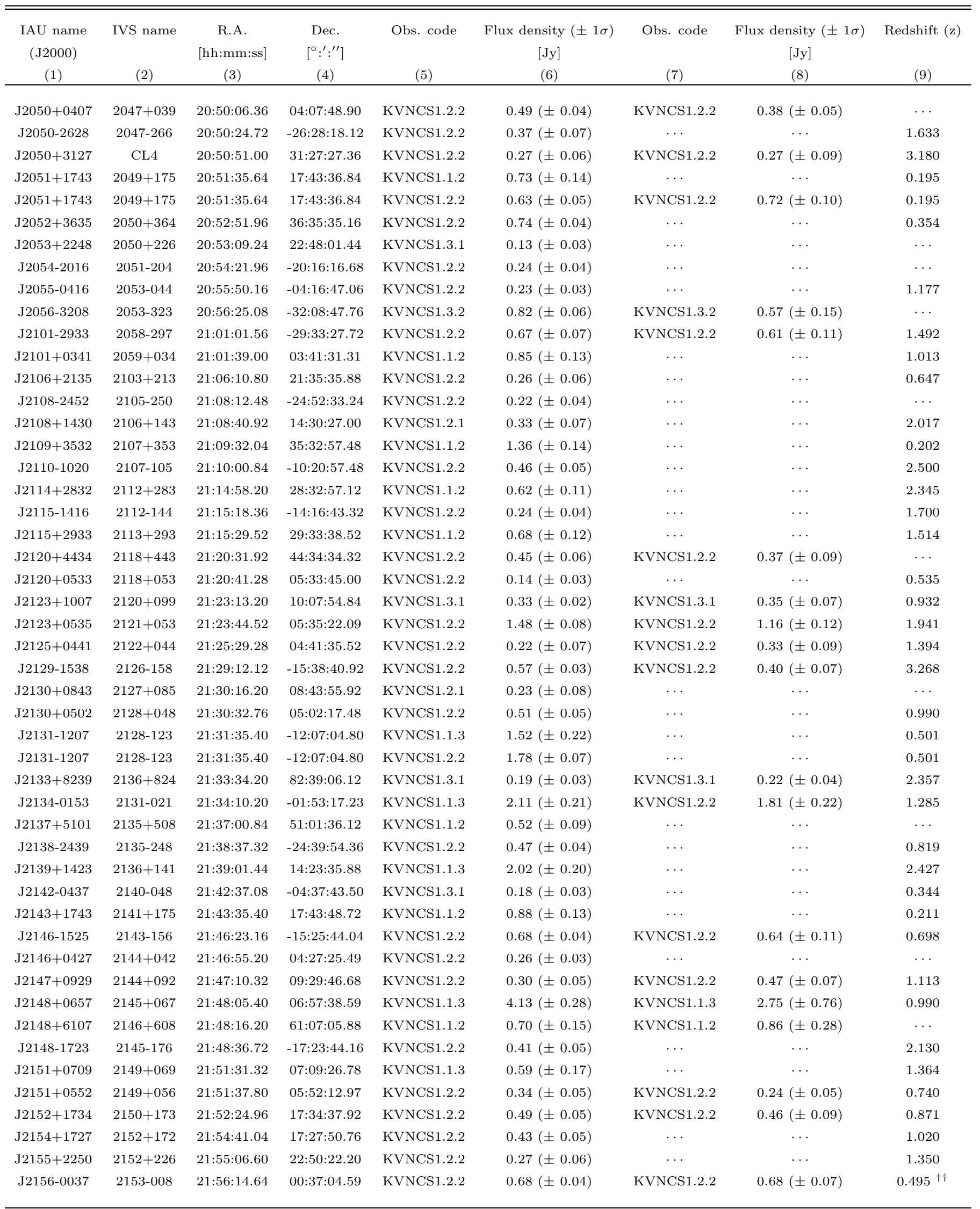


Table 2 (continued)

\begin{tabular}{|c|c|c|c|c|c|c|c|c|}
\hline $\begin{array}{l}\text { IAU name } \\
(\mathrm{J} 2000) \\
(1) \\
\end{array}$ & IVS name & $\begin{array}{c}\text { R.A. } \\
\text { [hh:mm:ss] } \\
(3) \\
\end{array}$ & $\begin{array}{c}\text { Dec. } \\
{\left[{ }^{\circ}:^{\prime}:^{\prime \prime}\right]} \\
(4) \\
\end{array}$ & $\begin{array}{l}\text { Obs. code } \\
(5) \\
\end{array}$ & $\begin{array}{c}\text { Flux density }( \pm 1 \sigma) \\
{[\mathrm{Jy}]} \\
(6) \\
\end{array}$ & Obs. code & $\begin{array}{c}\text { Flux density }( \pm 1 \sigma) \\
{[\mathrm{Jy}]} \\
(8) \\
\end{array}$ & Redshift ( $\mathrm{z}$ ) \\
\hline $\mathrm{J} 2157+1014$ & $2154+100$ & $21: 57: 12.96$ & $10: 14: 24.72$ & KVNCS1.2.1 & $0.39( \pm 0.10)$ & $\cdots$ & $\cdots$ & 0.761 \\
\hline $\mathrm{J} 2157+3127$ & $2155+312$ & $21: 57: 28.80$ & $31: 27: 01.44$ & KVNCS1.1.2 & $0.79( \pm 0.14)$ & $\cdots$ & $\cdots$ & 1.486 \\
\hline $\mathrm{J} 2158-1501$ & $2155-152$ & 21:58:06.24 & $-15: 01: 09.48$ & KVNCS1.1.3 & $1.67( \pm 0.15)$ & $\cdots$ & $\cdots$ & 0.672 \\
\hline $\mathrm{J} 2201+5048$ & $2159+505$ & $22: 01: 43.68$ & $50: 48: 56.52$ & KVNCS1.2.2 & $0.71( \pm 0.04)$ & KVNCS1.2.2 & $0.77( \pm 0.08)$ & 1.899 \\
\hline $\mathrm{J} 2202+4216$ & VR422201 & $22: 02: 43.44$ & $42: 16: 40.08$ & KVNCS1.1.2 & $5.21( \pm 0.28)$ & $\ldots$ & $\ldots$ & $\cdots$ \\
\hline $\mathrm{J} 2202-2335$ & $2200-238$ & 22:02:56.04 & $-23: 35: 10.32$ & KVNCS1.2.2 & $0.49( \pm 0.06)$ & KVNCS1.2.2 & $0.43( \pm 0.10)$ & 2.118 \\
\hline $\mathrm{J} 2203+6750$ & $2201+676$ & $22: 03: 12.60$ & $67: 50: 47.76$ & KVNCS1.2.2 & $0.27( \pm 0.04)$ & $\ldots$ & $\ldots$ & $\ldots$ \\
\hline $\mathrm{J} 2203+3145$ & $2201+315$ & 22:03:15.12 & $31: 45: 38.16$ & KVNCS1.1.2 & $3.31( \pm 0.23)$ & $\ldots$ & $\cdots$ & 0.295 \\
\hline $\mathrm{J} 2209+5158$ & $2207+517$ & 22:09:21.60 & 51:58:01.92 & KVNCS1.3.1 & $0.10( \pm 0.02)$ & $\cdots$ & $\cdots$ & $\cdots$ \\
\hline $\mathrm{J} 2209+3556$ & $2207+356$ & 22:09:45.36 & $35: 56: 00.96$ & KVNCS1.2.2 & $0.31( \pm 0.07)$ & KVNCS1.2.2 & $0.30( \pm 0.07)$ & 1.464 \\
\hline $\mathrm{J} 2211+1841$ & $2209+184$ & $22: 11: 53.88$ & $18: 41: 49.92$ & KVNCS1.2.2 & $0.53( \pm 0.05)$ & $\ldots$ & $\ldots$ & 0.070 \\
\hline $\mathrm{J} 2212+0819$ & $2209+080$ & $22: 12: 01.44$ & 08:19:16.50 & $\mathrm{KVNCS} 1.2 .2$ & $0.44( \pm 0.04)$ & KVNCS1.2.2 & $0.40( \pm 0.06)$ & 0.484 \\
\hline $\mathrm{J} 2212+2355$ & $2209+236$ & $22: 12: 06.12$ & $23: 55: 40.44$ & KVNCS1.1.2 & $0.92( \pm 0.13)$ & $\ldots$ & $\ldots$ & 1.125 \\
\hline $\mathrm{J} 2212+0646$ & $2210+065$ & $22: 12: 50.76$ & $06: 46: 08.72$ & KVNCS1.2.2 & $0.34( \pm 0.03)$ & KVNCS1.2.2 & $0.42( \pm 0.10)$ & 1.120 \\
\hline J2213-2529 & $2210-257$ & $22: 13: 02.64$ & $-25: 29: 30.12$ & $\mathrm{KVNCS} 1.2 .2$ & $0.76( \pm 0.05)$ & KVNCS1.2.2 & $0.50( \pm 0.09)$ & 1.833 \\
\hline $\mathrm{J} 2214+0711$ & $2211+069$ & $22: 14: 08.88$ & $07: 11: 42.40$ & KVNCS1.2.2 & $0.24( \pm 0.03)$ & $\ldots$ & $\ldots$ & $\cdots$ \\
\hline $\mathrm{J} 2217+2421$ & $2214+241$ & $22: 17: 00.96$ & $24: 21: 46.08$ & KVNCS1.2.2 & $0.89( \pm 0.04)$ & $\ldots$ & $\cdots$ & 0.505 \\
\hline $\mathrm{J} 2217+3156$ & $2215+316$ & $22: 17: 17.52$ & $31: 56: 49.92$ & KVNCS1.2.2 & $0.32( \pm 0.06)$ & KVNCS1.2.2 & $0.23( \pm 0.07)$ & $\ldots$ \\
\hline $\mathrm{J} 2218+1520$ & $2215+150$ & $22: 18: 10.80$ & $15: 20: 35.88$ & KVNCS1.1.3 & $0.75( \pm 0.20)$ & $\ldots$ & $\ldots$ & 2.335 \\
\hline J2230-1325 & $2227-136$ & $22: 30: 15.48$ & $-13: 25: 42.96$ & $\mathrm{KVNCS} 1.2 .2$ & $0.55( \pm 0.05)$ & KVNCS1.2.2 & $0.39( \pm 0.07)$ & 1.420 \\
\hline $\mathrm{J} 2230+6946$ & $2229+695$ & $22: 30: 36.36$ & $69: 46: 28.20$ & KVNCS1.2.2 & $0.57( \pm 0.10)$ & KVNCS1.2.2 & $0.34( \pm 0.09)$ & 1.413 \\
\hline $\mathrm{J} 2231-1422$ & $2228-146$ & $22: 31: 39.72$ & $-14: 22: 22.80$ & KVNCS1.3.1 & $0.35( \pm 0.03)$ & KVNCS1.3.1 & $0.32( \pm 0.08)$ & $\cdots$ \\
\hline $\mathrm{J} 2232-1659$ & $2229-172$ & $22: 32: 22.56$ & $-16: 59: 02.04$ & KVNCS1.2.2 & $0.28( \pm 0.04)$ & $\cdots$ & $\ldots$ & 1.780 \\
\hline $\mathrm{J} 2232+1143$ & CTA102 & $22: 32: 36.24$ & $11: 43: 50.88$ & KVNCS1.1.3 & $3.08( \pm 0.21)$ & KVNCS1.1.3 & $1.50( \pm 0.46)$ & 1.037 \\
\hline J2236-1706 & $2233-173$ & $22: 36: 09.36$ & $-17: 06: 21.96$ & $\mathrm{KVNCS} 1.2 .2$ & $0.26( \pm 0.03)$ & $\cdots$ & $\ldots$ & 0.647 \\
\hline $\mathrm{J} 2236+2828$ & $2234+282$ & $22: 36: 22.32$ & $28: 28: 57.36$ & KVNCS1.1.2 & $1.21( \pm 0.16)$ & $\cdots$ & $\cdots$ & 0.795 \\
\hline J2236-2309 & $2233-234$ & $22: 36: 26.28$ & $-23: 09: 26.64$ & KVNCS1.3.1 & $0.28( \pm 0.04)$ & $\ldots$ & $\ldots$ & $\ldots$ \\
\hline J2236-1433 & $2233-148$ & $22: 36: 34.20$ & $-14: 33: 22.32$ & KVNCS1.2.2 & $0.45( \pm 0.04)$ & KVNCS1.2.2 & $0.49( \pm 0.10)$ & 0.325 \\
\hline $\mathrm{J} 2238+2749$ & $2235+275$ & $22: 38: 12.84$ & $27: 49: 52.68$ & $\mathrm{KVNCS} 1.2 .2$ & $0.17( \pm 0.04)$ & $\cdots$ & $\ldots$ & 0.835 \\
\hline $\mathrm{J} 2238+1242$ & $2236+124$ & $22: 38: 34.44$ & $12: 42: 50.76$ & KVNCS1.2.2 & $0.25( \pm 0.03)$ & $\cdots$ & $\cdots$ & $0.335^{\dagger}$ \\
\hline $\mathrm{J} 2240+5133$ & $2238+512$ & $22: 40: 19.92$ & $51: 33: 11.88$ & KVNCS1.3.1 & $0.15( \pm 0.03)$ & $\cdots$ & $\cdots$ & $\cdots$ \\
\hline $\mathrm{J} 2241+4120$ & $2238+410$ & $22: 41: 07.08$ & $41: 20: 11.76$ & KVNCS1.2.1 & $0.33( \pm 0.11)$ & $\cdots$ & $\cdots$ & 0.726 \\
\hline $\mathrm{J} 2241+0953$ & $2239+096$ & $22: 41: 49.56$ & $09: 53: 52.44$ & KVNCS1.2.1 & $0.77( \pm 0.08)$ & $\cdots$ & $\cdots$ & 1.707 \\
\hline $\mathrm{J} 2243-2544$ & $2240-260$ & $22: 43: 26.40$ & $-25: 44: 30.84$ & KVNCS1.3.1 & $0.51( \pm 0.02)$ & $\cdots$ & $\cdots$ & 0.774 \\
\hline $\mathrm{J} 2244+4057$ & $2241+406$ & $22: 44: 12.84$ & $40: 57: 13.68$ & KVNCS1.2.1 & $0.91( \pm 0.13)$ & KVNCS1.2.1 & $0.82( \pm 0.27)$ & 1.171 \\
\hline $\mathrm{J} 2245+0324$ & $2242+031$ & $22: 45: 28.44$ & $03: 24: 08.86$ & $\mathrm{KVNCS} 1.2 .2$ & $0.27( \pm 0.07)$ & $\cdots$ & $\ldots$ & 1.340 \\
\hline $\mathrm{J} 2245-0755$ & 2243-081 & $22: 45: 48.96$ & $-07: 55: 19.38$ & KVNCS1.3.1 & $0.09( \pm 0.02)$ & $\cdots$ & $\cdots$ & $1.685^{\dagger}$ \\
\hline
\end{tabular}


Table 2 (continued)

\begin{tabular}{|c|c|c|c|c|c|c|c|c|}
\hline J2246-1206 & $2243-123$ & $22: 46: 18.12$ & $-12: 06: 51.12$ & KVNCS1.1.3 & $2.28( \pm 0.18)$ & KVNCS1.1.3 & $1.42( \pm 0.42)$ & 0.632 \\
\hline $\mathrm{J} 2247+0000$ & 2244-002 & $22: 47: 30.12$ & 00:00:06.46 & KVNCS1.1.3 & $0.67( \pm 0.14)$ & $\cdots$ & $\ldots$ & 0.094 \\
\hline $\mathrm{J} 2247-1237$ & $2245-128$ & $22: 47: 52.80$ & $-12: 37: 19.56$ & KVNCS1.2.2 & $0.48( \pm 0.05)$ & $\cdots$ & $\cdots$ & 1.892 \\
\hline $\mathrm{J} 2249+2107$ & $2246+208$ & $22: 49: 00.48$ & 21:07:03.00 & KVNCS1.2.2 & $0.45( \pm 0.04)$ & KVNCS1.2.2 & $0.30( \pm 0.07)$ & 1.274 \\
\hline $\mathrm{J} 2249-3039$ & 2246-309 & $22: 49: 19.20$ & $-30: 39: 12.60$ & KVNCS1.2.2 & $0.24( \pm 0.04)$ & $\ldots$ & $\ldots$ & 1.305 \\
\hline $\mathrm{J} 2249+1136$ & $2247+113$ & $22: 49: 54.48$ & $11: 36: 30.96$ & KVNCS1.2.1 & $0.36( \pm 0.08)$ & $\cdots$ & $\cdots$ & 0.026 \\
\hline $\mathrm{J} 2250+5550$ & $2248+555$ & $22: 50: 42.72$ & $55: 50: 14.64$ & KVNCS1.2.1 & $0.30( \pm 0.07)$ & $\cdots$ & $\cdots$ & $\cdots$ \\
\hline $\mathrm{J} 2253+1942$ & $2250+194$ & 22:53:07.44 & $19: 42: 34.56$ & KVNCS1.2.2 & $0.42( \pm 0.04)$ & $\cdots$ & $\cdots$ & 0.284 \\
\hline $\mathrm{J} 2254+1341$ & $2251+134$ & $22: 54: 20.88$ & $13: 41: 48.84$ & KVNCS1.1.3 & $0.87( \pm 0.13)$ & $\cdots$ & $\cdots$ & 0.673 \\
\hline J2255-0844 & $2252-089$ & $22: 55: 04.08$ & $-08: 44: 04.02$ & KVNCS1.2.2 & $0.23( \pm 0.03)$ & $\cdots$ & $\cdots$ & 0.606 \\
\hline $\mathrm{J} 2255+4202$ & $2253+417$ & $22: 55: 36.84$ & $42: 02: 52.44$ & KVNCS1.2.1 & $0.65( \pm 0.14)$ & KVNCS1.2.1 & $0.67( \pm 0.22)$ & 1.476 \\
\hline $\mathrm{J} 2256-2011$ & $2254-204$ & $22: 56: 41.28$ & $-20: 11: 40.56$ & KVNCS1.2.2 & $0.82( \pm 0.05)$ & KVNCS1.2.2 & $0.55( \pm 0.08)$ & $\ldots$ \\
\hline $\mathrm{J} 2257+0743$ & $2254+074$ & $22: 57: 17.28$ & $07: 43: 12.29$ & $\mathrm{KVNCS} 1.2 .2$ & $0.53( \pm 0.05)$ & KVNCS1.2.2 & $0.37( \pm 0.07)$ & 0.190 \\
\hline $\mathrm{J} 2257+0243$ & $2254+024$ & $22: 57: 17.64$ & 02:43:17.51 & KVNCS1.2.2 & $0.29( \pm 0.04)$ & KVNCS1.2.2 & $0.27( \pm 0.05)$ & 2.081 \\
\hline $\mathrm{J} 2258-2758$ & $2255-282$ & 22:58:05.88 & $-27: 58: 21.36$ & KVNCS1.1.3 & $2.32( \pm 0.23)$ & KVNCS1.1.3 & $1.53( \pm 0.40)$ & 0.926 \\
\hline J2258-2758 & $2255-282$ & $22: 58: 05.88$ & $-27: 58: 21.36$ & $\cdots$ & $\ldots$ & KVNCS1.2.2 & $3.52( \pm 0.35)$ & 0.926 \\
\hline $\mathrm{J} 2300+1655$ & $2258+166$ & $23: 00: 42.84$ & $16: 55: 14.52$ & $\mathrm{KVNCS} 1.2 .2$ & $0.64( \pm 0.05)$ & KVNCS1.2.2 & $0.53( \pm 0.08)$ & $\cdots$ \\
\hline J2301-0158 & $2258-022$ & 23:01:08.04 & $-01: 58: 04.58$ & KVNCS1.1.3 & $0.76( \pm 0.16)$ & $\cdots$ & $\cdots$ & 0.778 \\
\hline $\mathrm{J} 2301+3726$ & $2259+371$ & $23: 01: 27.84$ & $37: 26: 49.20$ & KVNCS1.2.2 & $0.50( \pm 0.07)$ & $\cdots$ & $\cdots$ & 2.179 \\
\hline $\mathrm{J} 2311+3425$ & $2308+341$ & $23: 11: 05.28$ & $34: 25: 10.92$ & KVNCS1.0.1 & $1.07( \pm 0.16)$ & $\ldots$ & $\cdots$ & 1.817 \\
\hline $\mathrm{J} 2311+3425$ & $2308+341$ & $23: 11: 05.28$ & $34: 25: 10.92$ & KVNCS1.2.2 & $1.16( \pm 0.07)$ & KVNCS1.2.2 & $1.11( \pm 0.11)$ & 1.817 \\
\hline $\mathrm{J} 2311+3425$ & $2308+341$ & $23: 11: 05.28$ & $34: 25: 10.92$ & KVNCS1.3.2 & $1.34( \pm 0.10)$ & KVNCS1.3.2 & $1.38( \pm 0.26)$ & 1.817 \\
\hline $\mathrm{J} 2311+4543$ & $2309+454$ & $23: 11: 47.40$ & $45: 43: 55.92$ & KVNCS1.1.2 & $0.61( \pm 0.11)$ & $\ldots$ & $\ldots$ & 1.447 \\
\hline $\mathrm{J} 2311+4543$ & $2309+454$ & $23: 11: 47.40$ & $45: 43: 55.92$ & KVNCS1.1.3 & $0.65( \pm 0.19)$ & $\cdots$ & $\cdots$ & 1.447 \\
\hline $\mathrm{J} 2312+7241$ & $2310+724$ & $23: 12: 19.80$ & $72: 41: 26.88$ & KVNCS1.2.2 & $0.27( \pm 0.05)$ & KVNCS1.2.2 & $0.31( \pm 0.05)$ & $\cdots$ \\
\hline $\mathrm{J} 2320+0513$ & $2318+049$ & $23: 20: 44.88$ & $05: 13: 49.94$ & KVNCS1.1.3 & $0.95( \pm 0.21)$ & $\cdots$ & $\cdots$ & 0.622 \\
\hline $\mathrm{J} 2321-0827$ & $2318-087$ & $23: 21: 18.36$ & $-08: 27: 21.53$ & KVNCS1.3.1 & $0.16( \pm 0.02)$ & $\cdots$ & $\cdots$ & 3.164 \\
\hline $\mathrm{J} 2321+3204$ & $2319+317$ & $23: 21: 55.08$ & $32: 04: 07.68$ & KVNCS1.1.2 & $0.54( \pm 0.10)$ & $\cdots$ & $\cdots$ & 1.489 \\
\hline $\mathrm{J} 2321+2732$ & $2319+272$ & $23: 21: 59.76$ & $27: 32: 46.32$ & $\mathrm{KVNCS} 1.2 .2$ & $0.70( \pm 0.03)$ & KVNCS1.2.2 & $0.66( \pm 0.08)$ & 1.253 \\
\hline $\mathrm{J} 2322+4445$ & $2319+444$ & $23: 22: 20.28$ & $44: 45: 42.48$ & KVNCS1.1.2 & $0.54( \pm 0.13)$ & $\cdots$ & $\ldots$ & $1.310^{\dagger \dagger}$ \\
\hline $\mathrm{J} 2322+5057$ & $2320+506$ & $23: 22: 26.04$ & $50: 57: 51.84$ & KVNCS1.0.1 & $1.37( \pm 0.16)$ & KVNCS1.0.1 & $1.43( \pm 0.37)$ & 1.279 \\
\hline $\mathrm{J} 2322+5057$ & $2320+506$ & $23: 22: 26.04$ & $50: 57: 51.84$ & $\ldots$ & $\ldots$ & KVNCS1.2.2 & $1.13( \pm 0.29)$ & 1.279 \\
\hline $\mathrm{J} 2322+5057$ & $2320+506$ & $23: 22: 26.04$ & $50: 57: 51.84$ & KVNCS1.3.2 & $1.29( \pm 0.08)$ & KVNCS1.3.2 & $1.24( \pm 0.23)$ & 1.279 \\
\hline $\mathrm{J} 2322+1843$ & $2319+184$ & $23: 22: 28.56$ & $18: 43: 24.96$ & KVNCS1.2.2 & $0.17( \pm 0.04)$ & $\cdots$ & $\ldots$ & 1.728 \\
\hline $\mathrm{J} 2322+0812$ & $2320+079$ & $23: 22: 36.12$ & 08:12:01.58 & KVNCS1.2.1 & $0.30( \pm 0.05)$ & $\cdots$ & $\cdots$ & 2.090 \\
\hline J2323-0150 & $2320-021$ & $23: 23: 04.56$ & $-01: 50: 48.12$ & KVNCS1.3.1 & $0.16( \pm 0.02)$ & $\ldots$ & $\cdots$ & 1.774 \\
\hline J2323-0317 & $2320-035$ & 23:23:31.92 & $-03: 17: 05.03$ & KVNCS1.2.2 & $0.96( \pm 0.04)$ & KVNCS1.2.2 & $0.84( \pm 0.09)$ & 1.410 \\
\hline
\end{tabular}


Table 2 (continued)

\begin{tabular}{|c|c|c|c|c|c|c|c|c|}
\hline $\begin{array}{l}\text { IAU name } \\
(\mathrm{J} 2000) \\
(1) \\
\end{array}$ & IVS name & $\begin{array}{c}\text { R.A. } \\
\text { [hh:mm:ss] } \\
(3) \\
\end{array}$ & $\begin{array}{c}\text { Dec. } \\
{\left[{ }^{\circ}:^{\prime}:^{\prime \prime}\right]} \\
(4)\end{array}$ & $\begin{array}{l}\text { Obs. code } \\
\qquad(5) \\
\end{array}$ & $\begin{array}{c}\text { Flux density }( \pm 1 \sigma) \\
{[\mathrm{Jy}]} \\
(6)\end{array}$ & Obs. code & $\begin{array}{c}\text { Flux density }( \pm 1 \sigma) \\
\qquad \mathrm{Jy}] \\
(8)\end{array}$ & Redshift (z) \\
\hline $\mathrm{J} 2327+1524$ & $2324+151$ & $23: 27: 21.96$ & $15: 24: 37.44$ & KVNCS1.2.2 & $0.28( \pm 0.04)$ & $\cdots$ & $\cdots$ & 0.046 \\
\hline $\mathrm{J} 2327+0940$ & $2325+093$ & $23: 27: 33.48$ & 09:40:09.48 & KVNCS1.1.3 & $0.97( \pm 0.13)$ & $\cdots$ & $\cdots$ & 1.843 \\
\hline $\mathrm{J} 2327+1533$ & $2325+152$ & $23: 27: 36.00$ & 15:33:09.72 & KVNCS1.2.2 & $0.27( \pm 0.03)$ & $\ldots$ & $\cdots$ & 0.988 \\
\hline J2327-1447 & $2325-150$ & $23: 27: 47.88$ & $-14: 47: 55.68$ & KVNCS1.2.2 & $0.43( \pm 0.04)$ & KVNCS1.2.2 & $0.28( \pm 0.07)$ & 2.465 \\
\hline $\mathrm{J} 2330+3348$ & $2327+335$ & $23: 30: 13.68$ & $33: 48: 36.36$ & KVNCS1.1.2 & $0.37( \pm 0.09)$ & $\cdots$ & $\cdots$ & 1.809 \\
\hline $\mathrm{J} 2330+3348$ & $2327+335$ & 23:30:13.68 & $33: 48: 36.36$ & KVNCS1.2.2 & $0.37( \pm 0.05)$ & $\cdots$ & $\cdots$ & 1.809 \\
\hline $\mathrm{J} 2330+3155$ & $2328+316$ & $23: 30: 46.08$ & $31: 55: 33.60$ & KVNCS1.3.1 & $0.20( \pm 0.03)$ & $\cdots$ & $\cdots$ & $\cdots$ \\
\hline J2331-1556 & $2329-162$ & $23: 31: 38.64$ & $-15: 56: 57.12$ & KVNCS1.1.3 & $0.75( \pm 0.22)$ & $\cdots$ & $\cdots$ & 1.153 \\
\hline $\mathrm{J} 2332+0838$ & $2330+083$ & $23: 32: 57.48$ & $08: 38: 10.43$ & KVNCS1.2.1 & $0.42( \pm 0.08)$ & $\cdots$ & $\cdots$ & $\cdots$ \\
\hline J2333-0131 & $2330-017$ & $23: 33: 16.56$ & $-01: 31: 07.39$ & KVNCS1.3.1 & $0.18( \pm 0.02)$ & $\cdots$ & $\cdots$ & 1.062 \\
\hline J2333-2343 & $2331-240$ & $23: 33: 55.08$ & $-23: 43: 40.80$ & KVNCS1.1.3 & $1.10( \pm 0.20)$ & $\ldots$ & $\ldots$ & 0.048 \\
\hline $\mathrm{J} 2334+0736$ & $2331+073$ & $23: 34: 12.72$ & $07: 36: 27.54$ & KVNCS1.1.3 & $1.04( \pm 0.13)$ & KVNCS1.1.3 & $1.23( \pm 0.32)$ & 0.401 \\
\hline J2335-2907 & $2332-293$ & $23: 35: 18.60$ & $-29: 07: 16.68$ & KVNCS1.3.1 & $0.28( \pm 0.02)$ & $\cdots$ & $\ldots$ & 0.931 \\
\hline J2335-0131 & $2332-017$ & $23: 35: 20.40$ & $-01: 31: 09.59$ & KVNCS1.3.1 & $0.41( \pm 0.02)$ & $\cdots$ & $\cdots$ & 1.184 \\
\hline J2337-1752 & $2335-181$ & $23: 37: 56.64$ & $-17: 52: 20.28$ & KVNCS1.3.1 & $0.30( \pm 0.03)$ & $\cdots$ & $\cdots$ & 1.446 \\
\hline J2337-0230 & $2335-027$ & $23: 37: 57.36$ & $-02: 30: 57.64$ & KVNCS1.1.3 & $0.69( \pm 0.17)$ & KVNCS1.1.3 & $0.98( \pm 0.30)$ & 1.072 \\
\hline $\mathrm{J} 2339+6010$ & $2336+598$ & $23: 39: 21.24$ & $60: 10: 12.00$ & KVNCS1.3.1 & $0.35( \pm 0.03)$ & $\cdots$ & $\cdots$ & $\cdots$ \\
\hline $\mathrm{J} 2340+2641$ & $2337+264$ & $23: 40: 28.92$ & $26: 41: 56.76$ & KVNCS1.2.2 & $0.41( \pm 0.04)$ & $\cdots$ & $\cdots$ & 0.372 \\
\hline $\mathrm{J} 2341+1928$ & $2338+191$ & $23: 41: 18.96$ & $19: 28: 05.52$ & KVNCS1.3.1 & $0.22( \pm 0.02)$ & $\cdots$ & $\cdots$ & 1.780 \\
\hline $\mathrm{J} 2343+2339$ & $2340+233$ & $23: 43: 12.36$ & $23: 39: 45.72$ & KVNCS1.3.1 & $0.22( \pm 0.03)$ & $\cdots$ & $\cdots$ & 1.328 \\
\hline $\mathrm{J} 2343+1543$ & $2341+154$ & $23: 43: 42.60$ & $15: 43: 03.00$ & KVNCS1.3.1 & $0.11( \pm 0.02)$ & $\cdots$ & $\cdots$ & 1.446 \\
\hline $\mathrm{J} 2346+0930$ & $2344+09 \mathrm{~A}$ & $23: 46: 36.84$ & $09: 30: 45.50$ & KVNCS1.2.2 & $0.73( \pm 0.06)$ & KVNCS1.2.2 & $0.46( \pm 0.06)$ & 0.677 \\
\hline $\mathrm{J} 2347+2719$ & $2345+270$ & $23: 47: 35.16$ & $27: 19: 01.56$ & KVNCS1.3.1 & $0.13( \pm 0.03)$ & $\cdots$ & $\cdots$ & 0.646 \\
\hline J2348-1631 & $2345-167$ & $23: 48: 02.52$ & $-16: 31: 12.00$ & KVNCS1.1.3 & $1.57( \pm 0.18)$ & KVNCS1.1.3 & $1.68( \pm 0.43)$ & 0.576 \\
\hline $\mathrm{J} 2348-0425$ & $2345-047$ & $23: 48: 11.88$ & $-04: 25: 56.39$ & KVNCS1.2.2 & $0.29( \pm 0.04)$ & KVNCS1.2.2 & $0.33( \pm 0.09)$ & 1.106 \\
\hline $\mathrm{J} 2349+3849$ & $2346+385$ & $23: 49: 21.00$ & $38: 49: 17.40$ & KVNCS1.0.1 & $0.58( \pm 0.15)$ & $\cdots$ & $\cdots$ & 1.032 \\
\hline $\mathrm{J} 2349+3849$ & $2346+385$ & $23: 49: 21.00$ & $38: 49: 17.40$ & KVNCS1.1.2 & $0.58( \pm 0.07)$ & $\cdots$ & $\cdots$ & 1.032 \\
\hline $\mathrm{J} 2350+1106$ & $2347+108$ & 23:50:02.04 & $11: 06: 36.72$ & KVNCS1.3.1 & $0.25( \pm 0.03)$ & $\cdots$ & $\cdots$ & 0.921 \\
\hline $\mathrm{J} 2352+7044$ & $2350+704$ & $23: 52: 52.68$ & $70: 44: 48.48$ & KVNCS1.3.1 & $0.14( \pm 0.03)$ & KVNCS1.3.1 & $0.16( \pm 0.04)$ & $\cdots$ \\
\hline $\mathrm{J} 2353+5518$ & $2351+550$ & $23: 53: 42.36$ & $55: 18: 40.68$ & KVNCS1.3.2 & $0.14( \pm 0.02)$ & $\cdots$ & $\cdots$ & 1.927 \\
\hline $\mathrm{J} 2354+4553$ & $2351+456$ & $23: 54: 21.60$ & $45: 53: 04.20$ & KVNCS1.0.1 & $0.65( \pm 0.21)$ & $\cdots$ & $\cdots$ & 1.992 \\
\hline $\mathrm{J} 2354+4553$ & $2351+456$ & $23: 54: 21.60$ & $45: 53: 04.20$ & KVNCS1.1.2 & $0.75( \pm 0.08)$ & $\cdots$ & $\cdots$ & 1.992 \\
\hline J2354-1513 & $2351-154$ & $23: 54: 30.24$ & $-15: 13: 11.28$ & KVNCS1.2.2 & $0.67( \pm 0.04)$ & $\cdots$ & $\cdots$ & 2.668 \\
\hline J2357-0152 & 2354-021 & $23: 57: 25.20$ & $-01: 52: 15.49$ & KVNCS1.2.2 & $0.19( \pm 0.05)$ & $\cdots$ & $\cdots$ & 0.812 \\
\hline J2357-1125 & $2354-117$ & $23: 57: 31.32$ & $-11: 25: 39.00$ & KVNCS1.1.3 & $0.94( \pm 0.09)$ & $\cdots$ & $\cdots$ & 0.960 \\
\hline J2358-1020 & 2355-106 & $23: 58: 10.92$ & $-10: 20: 08.52$ & KVNCS1.1.3 & $0.67( \pm 0.15)$ & $\cdots$ & $\cdots$ & 1.636 \\
\hline $\mathrm{J} 2358+3922$ & $2356+390$ & $23: 58: 59.88$ & $39: 22: 28.20$ & KVNCS1.2.1 & $0.56( \pm 0.11)$ & $\cdots$ & $\cdots$ & 1.198 \\
\hline $\mathrm{J} 2359+3850$ & $2356+385$ & $23: 59: 33.00$ & $38: 50: 42.36$ & KVNCS1.2.2 & $0.40( \pm 0.03)$ & $\cdots$ & $\cdots$ & 2.704 \\
\hline J2359-3133 & $2357-318$ & $23: 59: 35.52$ & $-31: 33: 43.92$ & KVNCS1.2.2 & $0.52( \pm 0.04)$ & $\cdots$ & $\cdots$ & 0.990 \\
\hline
\end{tabular}

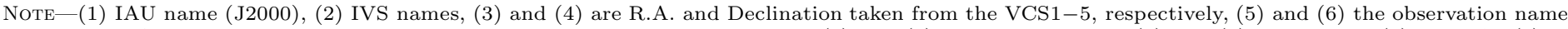

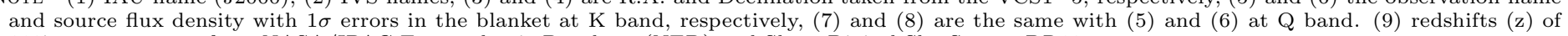
1137 sources, come from NASA/IPAC Extragalactic Database (NED) and Sloan Digital Sky Survey DR13.

* These redshift are taken from SDSS DR13.

$\dagger$ The $\mathrm{z}$-flag of these sources in NED are PHOT which means the photometric redshift.

$\dagger^{\dagger}$ The $\mathrm{z}$-flag of these sources in NED are UNCERTAIN which means the uncertain redshift. 